\title{
Random Walkers in 1-D Random Environments: Exact Renormalization Group Analysis
}

\author{
Daniel S. Fisher \\ Lyman Laboratory of Physics, Harvard University, Cambridge MA 02138, USA \\ Pierre Le Doussal \\ CNRS-Laboratoire de Physique Théorique de l'Ecole \\ Normale Supérieure, 24 rue Lhomond, F-75231 Paris \\ Cécile Monthus \\ Laboratoire de Physique Théorique et Modèles Statistiques \\ CNRS-Université Paris XI, Bât. 100, 91405 Orsay, France
}

\begin{abstract}
Sinai's model of diffusion in one-dimension with random local bias is studied by a real space renormalization group which yields exact results at long times. The effects of an additional small uniform bias force are also studied. We obtain analytically the scaling form of the distribution of the position $x(t)$ of a particle, the probability of it not returning to the origin and the distributions of first passage times, in an infinite sample as well as in the presence of a boundary and in a finite but large sample. We compute the distribution of meeting time of two particles in the same environment. We also obtain a detailed analytic description of the thermally averaged trajectories by computing quantities such as the joint distribution of the number of returns and of the number of jumps forward. These quantities obey multifractal scaling, characterized by generalized persistence exponents $\theta(g)$ which we compute. In the presence of a small bias, the number of returns to the origin becomes finite, characterized by a universal scaling function which we obtain. The full statistics of the distribution of successive times of return of thermally averaged trajectories is obtained, as well as detailed analytical information about correlations between directions and times of successive jumps. The two time distribution of the positions of a particle, $x(t)$ and $x\left(t^{\prime}\right)$ with $t>t^{\prime}$, is also computed exactly. It is found to exhibit "aging" with several time regimes characterized by different behaviors. In the unbiased case, for $t-t^{\prime} \sim t^{\prime \alpha}$ with $\alpha>1$, it exhibits a $\frac{\ln t}{\ln t^{\prime}}$ scaling, with a singularity at coinciding rescaled positions $x(t)=x\left(t^{\prime}\right)$. This singularity is a novel feature, and corresponds to particles which remain in a renormalized valley. For closer times $\alpha<1$, the two time diffusion front exhibits a quasi-equilibrium regime with a $\ln \left(t-t^{\prime}\right) / \ln t^{\prime}$ behavior which we compute. The crossover to
\end{abstract}


a $t / t^{\prime}$ aging form in the presence of a small bias is also obtained analytically. Rare events corresponding to intermittent splitting of the thermal packet between separated wells which dominate some averaged observables, are also characterized in detail. Connections with the Green's function of a one-dimensional Schrödinger problem and quantum spin chains are discussed. 


\section{Contents}

I Introduction 4

A Outling ........................... 7

II Models and real space renormalization procedure $\quad 8$

A Diffusion models . . . . . . . . . . . . . . . . . . 8

B $\quad$ Renormalization method . . . . . . . . . . . . . . . . . . 9

1 Definitions and RG equations . . . . . . . . . . . . . . . . . . 9

$2 \quad$ Effective dynamics and validity of the method . . . . . . . . . . . 12

3 RG with one boundary: reflecting or absorbing . . . . . . . . . 14

C General analysis of the RSRG equations . . . . . . . . . . . . . . 15

1 Symmetric model. . . . . . . . . . . . . . . . . . . 15

2 Biased model . . . . . . . . . . . . . . . . . . . . 16

3 Boundary fixed point solutions . . . . . . . . . . . . . . . . . 19

III Single time diffusion properties in Sinai model 19

A Single time diffusion front for the symmetric model . . . . . . . . . 19

B $\quad$ Single time diffusion front for the biased model . . . . . . . . . . . . 20

IV Motion of thermal averages: returns to the origin and jumps 22

A Number of returns to the origin: symmetric case . . . . . . . . 23

B Distribution of the sequence of returns to the origin: symmetric case 26

C Number of jumps up to time $t$ for the effective dynamics . . . . . . 27

D Correlations of the jumps . . . . . . . . . . . . . . 28

E Number of returns to the origin: biased case . . . . . . . . . . . . 29

F Distribution of the sequence of returns to the origin: biased case . 31

$\mathrm{V} \quad$ Return to the origin of a single walker, first passage times and $\begin{array}{ll}\text { meeting time of two walkers } & 32\end{array}$

A Probability of no return to the origin for a single walken . . . . . . 32

B $\quad$ First passage times in an infinite sample . . . . . . . . . . . . . . 33

1 Symmetric case . . . . . . . . . . . . . . . . . . . . . . 34

2 Biased case . . . . . . . . . . . . . . . . . . . 35

C Distribution of the maximum position . . . . . . . . . . . . 36

D $\quad$ Probability that two particules do not meet up to time $t$. . . . . . 36

VI Two time diffusion front in the Sinai model and aging properties 37

A Discussion of the various regimes . . . . . . . . . . . . . 38

B Singular part of the two time diffusion front: symmetric case . . . 40

1 Probability $D_{\alpha}$ of staying within a well from $t^{\prime}$ to $t$. . . . . . 40

2 Weight $D_{\alpha}\left(\tilde{X}^{\prime}\right)$ of the delta function component of $P_{\alpha}\left(X, \tilde{X}^{\prime}\right) .41$

C Probability of staying within a well: biased case . . . . . . . . . . 42

D $\quad$ Two-time diffusion front: full analysis . . . . . . . . . . . . . . . 43

1 Sketch of the method . . . . . . . . . . . . . . . . . . 43

2 Some results for the symmetric case . . . . . . . . . . . . . . . 44

3 Some results for the biased case . . . . . . . . . . . . . . . . . 46

E $\quad$ Full two-time aging function in a semi-infinite system . . . . . . . 46 
F Dynamics within a well . . . . . . . . . . . . . . . . . . . 48

G crossover at $t \sim t$. . . . . . . . . . . . . . . 50

H Rare events in the single time-diffusion front . . . . . . . . . 51

\begin{tabular}{|lll}
\hline VII Finite size properties of Sinai's model & 54
\end{tabular}

A $\quad$ RG for a finite size system . . . . . . . . . . . . . . . . . 54

B Evolution towards equilibrium in a system with reflecting boundaries 56

1 Distribution of equilibration time . . . . . . . . . . 56

2 Distribution of equilibrium position $x_{e q}$. . . . . . . . . . . . . 57

C First passage times. . . . . . . . . . . . . . . 57

1 With a reflecting boundary . . . . . . . . . . . . . . . 57

2 With an absorbing boundary . . . . . . . . . . . . . . . . 58

D Averaged diffusion front . . . . . . . . . . . . . . . . . 59

VIII Results for Fokker Planck and associated Schrödinger operator 59

A From Fokker Planck to Schrödinger operator . . . . . . . . . . 60

B $\quad$ Averaged Green's function for the Schrödinger operator . . . . . . 60

$\begin{array}{lll}\text { IX } & \text { conclusions } & 61\end{array}$

\begin{tabular}{ll}
\hline APPENDIXES & 63
\end{tabular}

\begin{tabular}{|lll}
\hline A Auxiliary variable RG rule, symmetric case & 63
\end{tabular}

B Auxiliary variable RG rule, biased case 64

C Correlation of times and directions of successive jumps 65

1 Conditional probabilities of times of jumps forward and backward. 65

2 Correlations in the sequence of times of successive forward and backward jumps ................ 66

$\mathrm{D}$ distribution of sequences of returns to the origin: biased case 66

\begin{tabular}{lll}
\hline E dynamics within a well & 67
\end{tabular}

$1 \quad$ Probability that a bond has degenerate minima . . . . . . . . . . . 67

$2 \quad$ Relationship to the associated Schrödinger operator Green's function 69

\begin{tabular}{|ll}
\hline F & Solution of the two time RG equations
\end{tabular}

G Disorder averaged probability distribution for a finite size system

\section{INTRODUCTION}

Studying non-equilibrium dynamics provides a useful route to elucidate the properties of systems with quenched disorder. In addition it is very relevant for experiments, since most such systems form glassy states with ultraslow dynamics and usually do not reach full thermal equilibrium within the accessible time scales. This 
is the case for a variety of experimental systems such spin glasses [1], random field systems [2,3], vortex lines in superconductors [4,5], and domain growth in presence of quenched disorder. Despite decades of extensive work, there are still a number of unresolved issues in the theoretical description of the dynamics of systems with quenched disorder. This uncertainty is due, to a large extent, to the lack of physically relevant models for which analytical solutions can be obtained, providing clear cut answers to well posed questions. The need for such models is all the more acute since it is prohibitively difficult to obtain unambiguous answers from numerical simulations when the dynamics is ultraslow, especially since the interpretation is often blurred by the absence of precise theoretical predictions. Solvable models, where the answers are known, should also provide useful testing ground for numerical methods by giving clues on the necessary simulation time scales and averaging procedures in disordered systems which are often dominated by rare events.

Some progress has been made in obtaining analytical solutions for the large time behavior of mean field type models [6]. Although it is still extremely unclear how much these mean field results will carry through to short range, finite dimensional systems, one outcome of these works [6] 8 ] has been to demonstrate the existence of several possible large time regimes and to attempt to classify them. This provides further motivation to study aging dynamics in a larger class of models, in particular to study the possible ways of taking the large time $t, t^{\prime} \rightarrow \infty$ limits for correlations between configurations of the system at a waiting time $t^{\prime}=t_{w}$ after a quench at $t=0$, and a later observation time $t$.

Other types of approaches, such as droplet descriptions of the statics and the non-equilibrium dynamics of disordered systems [3], make use of domain growth arguments. These approaches emphasize the leading role of thermally activated processes, which should play an important role in short range models, while mean field dynamics may be dominated by other type of collective processes [9]. The "coarsening" of domain structures evolving towards equilibrium has been studied extensively in pure models [10] but little is known rigorously for domain growth with quenched disorder. Thus these approaches are still to a large extent phenomenological and one would like to find models where solid results about aging in the presence of activated dynamics can be obtained analytically. A natural hope for that would be to study 1D models which could be used as testing grounds for more complex $D>1$ cases which have resisted analytic attack.

A celebrated 1D toy model for glassy activated dynamics is the Sinai model, which describes the diffusion of a random walker in a 1D random static force fieldequivalent to a random potential which itself has the statistics of a 1D random walk [11]. Although this model (with or without a bias) has been much studied, the known analytical results [11 16] usually concern single time and single particle quantities and are technically hard to obtain. It is known that this model without a bias exhibits non trivial ultraslow logarithmic behavior, as the walker typically moves as $x \sim(\ln t)^{2}$, as well as several dynamical phases with anomalous diffusion as the bias is increased from zero. By contrast, there was until now no exact results about two time aging dynamics, despite several mostly qualitative and numerical studies [17, 16] which found interesting aging behavior in this model. In addition, the Sinai model has interesting extensions to many interacting particles, and via domain walls, to the Glauber dynamics of 1D random field Ising ferromagnets and 
spin glasses in a magnetic field.

Recently we have proposed an approach, based on a real space renormalization group (RSRG) method, which allows us to obtain many exact results for the non equilibrium dynamics of several 1D disordered systems [18]. We have shown that it applies to the Sinai model as well as to 1D disordered spin models and diffusionreaction processes in Sinai's type of energy landscapes. This RSRG method is closely related to that used to study disordered quantum spin chains [19 24. The crucial feature of the $\mathrm{RG}$ is coarse-graining the energy landscape in a way that preserves the long time dynamics. In Sinai's model the way to implement the RSRG is very direct: one decimates iteratively the smallest energy barrier in the system stopping when the time to surmount the smallest remaining barrier is of order the time scale of interest. Despite its approximate character, the RSRG yields for many quantities asymptotically exact results. As in 21] it works because the iterated distribution of barriers grows infinitely wide, consistent with [11].

The aim of the present paper is to show in detail how the RSRG method applies to the Sinai model, allowing one to obtain in a simple way a large number of exact results. We obtain a host of quantities such as return and first passage probabilities, single time correlations as well as two time correlations of the type that are probed in aging experiments. Given the long history of Sinai's model, some of the results obtained here have been derived previously, by completely different methods. These methods include those from probability theory [25,11,26 28, as well as conventional methods of the physics of disordered systems, such as the Dyson Schmidt method, replica methods, supersymmetry, transfer matrix etc. [29]. Despite that, a large number of our results are, to our knowledge, novel. Indeed, as we aim to illustrate in this paper, the most interesting feature of the RSRG, besides being simple to apply, is that it allows one to obtain all these results (new and old) from a single method, while other methods usually allow access to only specific types of exact results. As we will explain, the only limitations are the ones usually associated with any RG method. First, almost by definition, it only addresses and obtains exactly the universal quantities, i.e., the ones which are independent of the short scale details of the model. Second, it does rely on the global assumption that the starting model is within the basin of attraction of the zero temperature fixed point studied here, and is thus not "exact from first principles". This is not a restriction in the case of the Sinai model: because rigorous results already exist from probability theory, this last assumption can be considered established.

In two companion papers [30, 31], we will detail the applications of the RSRG, given as a short account in [18], to the Glauber dynamics of disordered spin models and to diffusion-reaction processes in presence of quenched disorder. These works rely heavily on the Sinai model and the present work. Thus we here give a detailed presentation of the results for Sinai model.

An interesting feature of the RSRG is that it demonstrates in a simple and operational way how the Sinai model is related to other one-dimensional disordered models. More formal derivations of such mappings can also be made in some cases via free fermion models. For instance, the quantum XX spin chains with disorder and the random transverse field Ising chain (RTFIC) are related via Jordan Wigner transformations to free fermion models near half filling with disorder in the hopping term. This problem is in turn related, via its expression as a random Dirac prob- 
lem, to a supersymmetric random Schrödinger operator 32 identical to the Fokker Planck diffusion operator associated with the Sinai model 14,33. Most of these relations have been detailed previously in various contexts (see, e.g. for a review [34 36]). These disordered fermion models have been much reinvestigated recently as they provide examples of quantum delocalization transitions. It may sometimes be useful to recast them in terms of the Sinai model where some quantities have a straightforward physical interpretation (e.g., the logarithmic Arrhenius diffusion over barriers growing as a random walk gives the logarithmic energy dependence of the local density of states). The RSRG demonstrates such mappings for the low energy (large time) properties in a very direct way, as we will illustrate. Zero drift in Sinai's model corresponds to the self dual critical case in the RTFIC [21] and to the antiferromagnetic XX chain [20,19], while the zero velocity biased phase [13, 14] corresponds to the Griffiths phase of the RTFIC [21] and a dimerized XX chain. As we show here, magnetization properties correspond to persistence properties in Sinai's model.

Although the idea of studying random diffusion problem via real space decimation techniques has been used previously, it has been mostly applied to fractal or hierarchical landscapes (see e.g. [37]) which are designed for such methods. By contrast, here the RSRG emerges from the structure of the zero temperature fixed point itself, as the natural way to treat diffusion in a statistically translationally invariant disordered system, with no ad-hoc assumptions. Interestingly, a similar property arises in the problem of the coarsening of the pure $1 \mathrm{D} \Phi^{4}$ model at zero temperature, which can be treated exactly by successive elimination of the smallest domains in the system [38], a method reminiscent of the RSRG studied here. Finally note that since 18 appeared, several new papers have been devoted to the Sinai model [39 42].

\section{A. Outline}

The outline of this paper is as follows. Section II contains a pedagogical introduction to the real space renormalization (RSRG) approach for the Sinai model. It terminates with the explicit expressions for the fixed points of the RSRG ([IC). In Section III we compute the averaged single time diffusion front for the symmetric Sinai model in ([IIA) and with a bias in (IIIB). In Section [V we study the returns to the origin (persistence properties) of the thermally averaged motion, as well as the statistics of the jumps, in the symmetric and biased case. In Section $\nabla$, we study returns to the origin of a single walker, distributions of first passage time and of the maximum position as well as the probability of meeeting of two walkers. Section VI is devoted to the aging properties of the Sinai model and contains a general discussion (VIA $)$, calculations of singular parts of the diffusion front (VIB $)$, the full two-time probability distribution (VID), and the analysis of a simpler case (VID 2). The section terminates with the analysis of rare events and calculation of the front in the quasi-equilibrium regime $(\nabla T \mathrm{~F})$ and fluctuations in the single time diffusion front. In Section VIIA the RSRG is studied in a finite size system; equilibration properties, first passage times with boundaries and finite size diffusion fronts are computed. Finally in section VIII we obtain the Green's function of the 
associated Schrödinger operators in VIIIB. Section [X contains the conclusions. Further technicalities are relegated to various Appendices.

\section{MODELS AND REAL SPACE RENORMALIZATION PROCEDURE}

\section{A. Diffusion models}

Diffusion in one-dimensional random media has been modeled in three ways, which usually lead to equivalent classes of behavior in the large time limit. Probabilists have often studied models discrete in time and space; for instance, a particle on points of a one-dimensional lattice, $n$, which jumps to the right $(n+1)$ with probability $p_{n}$ and to the left with probability $1-p_{n}$. Physicists on the other hand have often considered random hopping models, continuous in time but discrete in space, described by the master equation:

$$
\frac{d P_{n}(t)}{d t}=-\left(J_{n+1, n}-J_{n, n-1}\right) \quad J_{n+1, n}=W_{n+1, n} P_{n}-W_{n, n+1} P_{n+1}
$$

$W_{n+1, n}$ and $J_{n+1, n}$ are respectively the transition rate and the current from $n$ to $n+1$, and $W_{n, n+1}$ and $J_{n, n+1}=-J_{n+1, n}$ from $n+1$ to $n$. Finally fully continuum models, with Fokker Planck equation:

$$
\partial_{t} P(x, t)=H_{F P} P=\partial_{x} D(x)\left(T \partial_{x} P-F(x) P\right)
$$

have also been studied (with $D(x)>0$ ).

It is useful to distinguish three classes of disorder (within each description) leading to different types of generic 1D large time behavior (for uncorrelated disorder).

(i) detailed balance, random diffusion coefficient

This corresponds to $W_{n, n+1}=W_{n+1, n} \equiv D_{n, n+1}$ in (1) or to $F(x)=0$ and $D(x)$ a random positive function. It is well known that the large time diffusion coefficient is $D_{\text {eff }}=\langle 1 / W\rangle^{-1}$ for uncorrelated disorder and thus that this model exhibits asymptotic "normal diffusion" unless the $D_{n, n+1}$ have a broad distribution, with a tail near the origin, $P(D) \sim D^{-\alpha}(0 \leq \alpha<1)$.

(ii) random traps

This corresponds to $W_{n, n+1}=1 / \tau_{n+1}$ and $W_{n+1, n}=1 / \tau_{n}$. Each site is characterized by a release time, but the exit is with the same probability $1 / 2$ to the left or to the right (the jump probability depends only on the starting point). Again this model exhibits asymptotic "normal diffusion" unless the release times have an algebraically broad distribution.

(iii) generic case: Sinai model

In the generic case one can always parametrize the hopping rates as:

$$
W_{n, n+1}=\tau_{0}^{-1} e^{\beta E_{n, n+1}} e^{-\frac{1}{2} \beta\left(U_{n}-U_{n+1}\right)} \quad W_{n+1, n}=\tau_{0}^{-1} e^{\beta E_{n, n+1}} e^{-\frac{1}{2} \beta\left(U_{n+1}-U_{n}\right)}
$$

where $\beta=1 / T$ and $T$ is the temperature. This can be illustrated as in Fig. 1: there is a symmetric barrier $E_{n, n+1}$ between sites $n$ and $n+1$, plus an additional potential difference. The barrier $E_{n, n+1}$ gives the average diffusion coefficient (or attempt frequency) on the bond. The "forces" on the bonds are $f_{n+1, n}=-\left(U_{n+1}-U_{n}\right)$ which 
represent a local bias. In a finite size system (periodic in the $U_{n}$ ) the expressions (3) correctly lead to the Gibbs zero current equilibrium measure, $e^{-\beta U_{n}} / Z$.

The main case of interest here and studied by Sinai is that of independent random forces. The generic case, for uncorrelated disorder and for distributions of $f_{n}$ and $E_{n, n+1}$ with fast enough decay (e.g. faster than exponential) all belong to the class of Sinai's model, which is a discrete time model. A similar potential can be introduced for this model: $U_{n}-U_{0}=T \sum_{i=1}^{n-1} \log \left(p_{i} /\left(1-p_{i}\right)\right)$. One can most easily visualize these as Arrhenius motion in a random potential $U_{n}$ which itself performs a random walk, either symmetric or biased. This motion has been studied extensively and it is known that diffusion is logarithmic $x \sim \ln ^{2} t$ in the symmetric case, sublinear $x \sim t^{\mu}$ for a small bias $(\mu<1)$ and with a finite velocity $x \sim V t$ for $\mu>1$, where $\mu$ is related to the asymmetry of the force distributions as defined later.
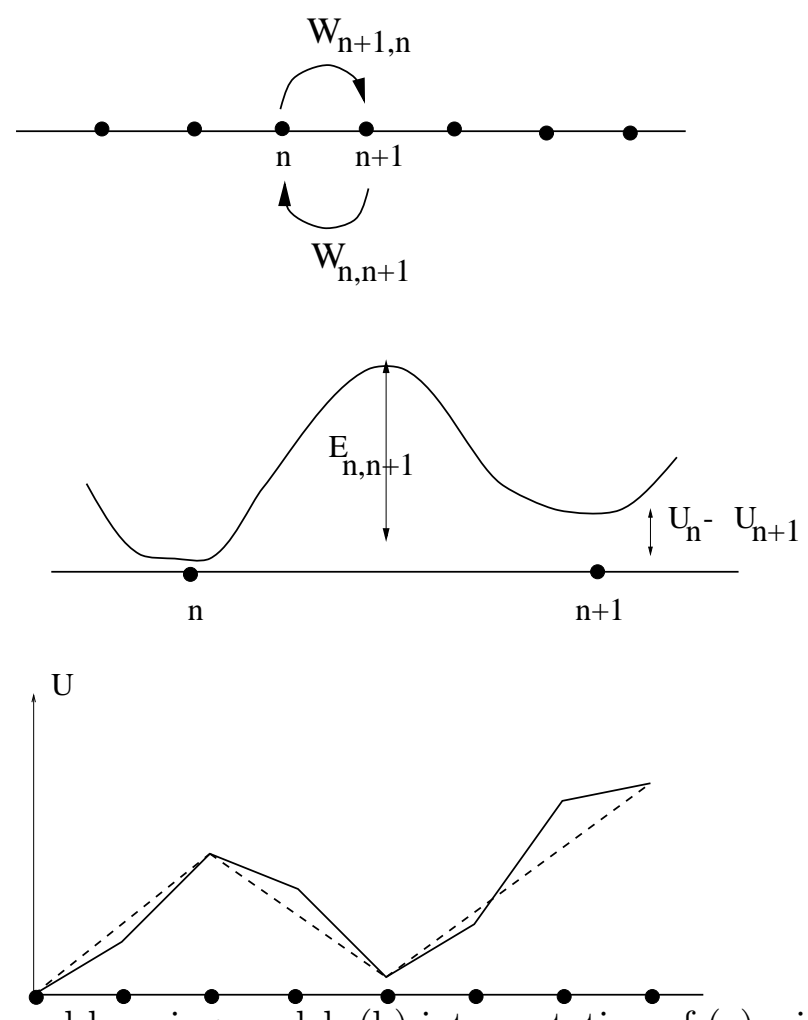

FIG. 1. (a) General hopping model; (b) interpretation of (a) with a barrier between each site and a potential difference (local bias); (c) model studied here.

For convenience and to be specific in what follows we will mostly study, as our basic model, the random hopping model with the choice $E_{n, n+1}=0$. We will also compare with the discrete time model originally studied by Sinai. However, our results are much more general and apply to any model within the locally random force class (with short range correlations).

\section{B. Renormalization method}

\section{Definitions and $R G$ equations}

As described above, we consider models of diffusion in 1D landscapes in which walkers perform Arrhenius diffusion in a potential $U_{n}$ ( $n$ is a site index). A "force" 
variable $f_{n}=U_{n}-U_{n+1}$ is defined on each bond $(n, n+1)$ (indexed as bond $n$ ) and as in the Sinai model, the $f_{n}$ are independent random variables with distribution $Q(f) d f$. The long-time dynamics in such landscapes are primarily determined by the large barriers and deep valleys. Thus we need to be able to focus on these aspects of the landscape while eliminating as much as possible the effects of the finer scale structure.

We therefore introduce a renormalization procedure, for a given landscape, which will allow us, in this way, to study the asymptotic dynamics. We should emphasize that we will apply it mainly to the case of forces independent from bond to bond, but it can in principle be applied to any 1D landscape. The crucial feature which is needed for the RG to yield asymptotically exact results, is that the landscapes have extremal values of the potential which grow with length scale. This will make the distributions of the renormalized barriers broader and broader. In the case of the Sinai model, it is possible to follow exactly the RG flow (because the forces remain uncorrelated under the $\mathrm{RG}$ ) and thus to check a posteriori that at large scales the distributions of renormalized barriers are indeed very broad. However, the procedure is much more general and would also lead to asymptotically exact results for correlated landscapes in which the renormalized barriers become higher and higher. The difficulty in such correlated cases is to follow the distributions. Of course there are 1D landscapes for which the RSRG would not give exact results for the diffusion behavior: in particular, bounded potentials which have normal diffusive behavior.

The RSRG procedure on a given landscape is implemented as follows. One can first group the bonds with the same sign of the force (see Fig. 1 c), and then can start, with no loss of generality, from an "antiferromagnetic" landscape (see Fig. 2) with the $f_{n}^{\prime}$ alternatively positive and negative) but with a distribution of bond lengths $l_{i}$. Our starting model is thus defined by $f_{n}^{\prime}=(-1)^{n} F_{n}$ where the $F_{n}=\left|U_{n}-U_{n+1}\right|$ are the useful variables - called here "barriers" - and the two bond variables $F, l$ are chosen independently from bond to bond with an initial distribution $P(F, l)$. In the presence of a bias one needs two distinct distributions $P^{+}(F, l)$ for "descending bonds" and $P^{-}(F, l)$ for "ascending bonds" (opposing the mean force), both normalized to unity. Note that the combining together of consecutive descending bonds in this way naturally leads to an exponential tail in the distribution $P^{-}$and likewise in $P^{+}$. Such exponential tails in barrier distributions will play an important role in the physics and in our analysis.

We are interested in long times when the behavior will be dominated by large barriers and it is on these that we must focus. Our RG procedure is conceptually simple: in a given energy landscape it consists of iterative decimation of the bond with the smallest barrier $\Gamma=F_{\min }$, say $F_{2}=U_{3}-U_{2}=\Gamma$ as illustrated in Fig. 2. At time scales much longer than $\exp \left(F_{2} / T\right)$, local equilibrium will be established between sites 2 and 3 and the rate for the walker to get from 4 to 1 will be essentially the same as it would be if sites 2 and 3 did not exist but 1 and 4 were instead connected by a bond with barrier

$$
F^{\prime}=F_{1}-F_{2}+F_{3}
$$

and length

$$
l^{\prime}=l_{1}+l_{2}+l_{3} .
$$


We thus carry out exactly this replacement. This preserves the zigzag structure (the model remains alternating "up-down") and the larger scale extrema of the potential since the total length and the extrema of $U$ in the segments are exactly preserved. Furthermore if the starting distribution is independent forces from bond to bond, this remains so under the RG. One then keeps on iteratively eliminating barriers $\Gamma<F<\Gamma+d \Gamma$ thereby gradually decreasing the minimum remaining barrier height, $\Gamma$. Note that there is no ambiguity in the case of continuous distributions as considered here, as one can always neglect the unlikely events when two neighbors or next nearest neighbors are within $d \Gamma$.

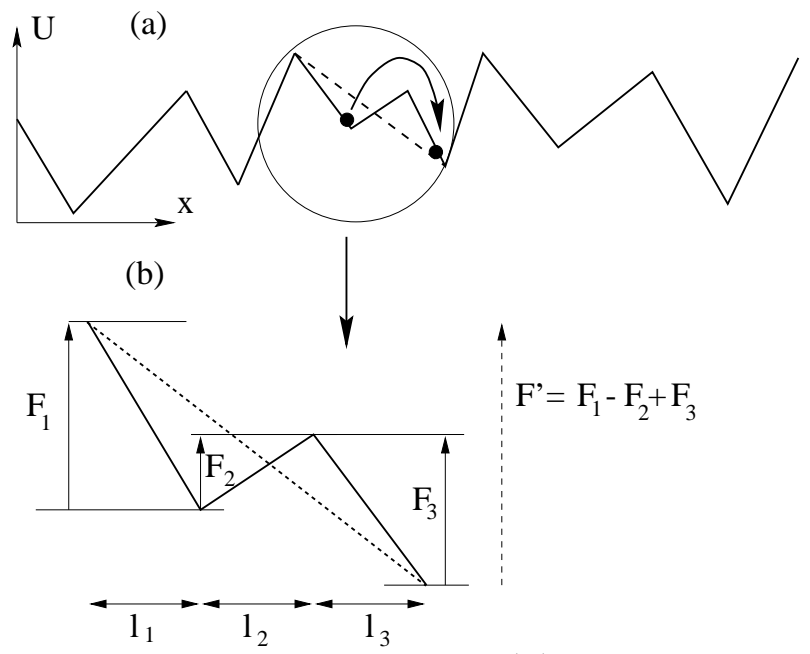

FIG. 2. (a) Energy landscape in Sinai model; (b) decimation method: the bond with the smallest barrier $F_{\min }=F_{2}$ is eliminated as three bonds are grouped into one (see text).

The above rules for $F$ and $l$ define the RSRG transformation for arbitrary landscapes. In the case of the Sinai landscape where bonds remain statistically independent one can define

$$
\zeta_{n} \equiv F_{n}-\Gamma
$$

and introduce $P_{\Gamma}^{+}(\zeta=F-\Gamma, l)$ and $P_{\Gamma}^{-}(\zeta=F-\Gamma, l)$ which denote the probabilities that a \pm renormalized bond at scale $\Gamma$ has a barrier $F=\Gamma+\zeta>\Gamma$ and a length $l$, each normalized by $\int_{0}^{\infty} d \zeta \int_{0}^{\infty} d l P_{\Gamma}^{ \pm}(\zeta, l)=1$. One can then explicitly write closed RG equations for these two distributions describing their evolution under the decimation represented in Fig. 2:

$$
\begin{aligned}
\left(\partial_{\Gamma}-\partial_{\zeta}\right) P_{\Gamma}^{ \pm}(\zeta, l)= & P_{\Gamma}^{\mp}(0, .) *_{l} P_{\Gamma}^{ \pm}(., .) *_{\zeta, l} P_{\Gamma}^{ \pm}(., .)-2 P_{\Gamma}^{ \pm}(\zeta, l) \int_{0}^{\infty} d l^{\prime} P_{\Gamma}^{\mp}\left(0, l^{\prime}\right) \\
& +P_{\Gamma}^{ \pm}(\zeta, l) \int_{0}^{\infty} d l^{\prime}\left(P_{\Gamma}^{ \pm}\left(0, l^{\prime}\right)+P_{\Gamma}^{\mp}\left(0, l^{\prime}\right)\right)
\end{aligned}
$$

where $*_{\zeta}$ denotes a convolution with respect to $\zeta$ only and $*_{\zeta, l}$ with respect to both $\zeta$ and $l$ with the variables to be convoluted denoted by dots. The first term on the r.h.s. represents the new renormalized bonds, the second the bonds which are decimated as neighbors of the smallest barrier and the last comes from keeping the distribution normalized. The total number $n_{\Gamma}$ of bonds in the system evolves as 


$$
\partial_{\Gamma} n_{\Gamma}=-n_{\Gamma} \int_{0}^{\infty} d l^{\prime}\left(P_{\Gamma}^{+}\left(0, l^{\prime}\right)+P_{\Gamma}^{-}\left(0, l^{\prime}\right)\right)
$$

We need also to introduce the average lengths

$$
\bar{l}_{\Gamma}^{ \pm}=\int_{0}^{\infty} d \zeta \int_{0}^{\infty} d l l P_{\Gamma}^{ \pm}(\zeta, l)
$$

of a \pm bond, and the total average length $\bar{l}_{\Gamma}=\bar{l}_{\Gamma}^{+}+\bar{l}_{\Gamma}^{-}$of a valley that evolves as

$$
\partial_{\Gamma} \bar{l}_{\Gamma}=\bar{l}_{\Gamma} \int_{0}^{\infty} d l^{\prime}\left(P_{\Gamma}^{+}\left(0, l^{\prime}\right)+P_{\Gamma}^{-}\left(0, l^{\prime}\right)\right)
$$

We have of course that $n_{\Gamma} \sim 1 / \bar{l}_{\Gamma}$.

The RG equations (6) derived here for Sinai's model are identical to those derived to study the low energy properties of the random transverse field Ising chain (RTFIC) in [21] (we choose notations and conventions as in [21]) using a perturbative analysis of the effects of the strongest bonds and fields. The reason for this is that the two models are in fact formally related, as mentionned in the Introduction. At the level of the RSRG equations, the mapping appears in a very simple way: the local random fields $h_{k}$ and the random exchanges $J_{k}$ in the RTFIC correspond to the ascending and descending barriers respectively, through the relations $F_{2 k} / T=-\ln h_{k}$ and $F_{2 k+1} / T=-\ln J_{k}$. We can also identify the renormalization scale $\Gamma$ in both models. For the diffusion model it corresponds to an Arrhenius time scale $t=t_{0} \exp (\Gamma / T)$ to go over a barrier $F=\Gamma$, whereas in the quantum model it corresponds to the minimal energy scale of the levels which have been eliminated $\Omega=\Omega_{0} e^{-\Gamma}$. The duality between $J$ and $h$ in the RTFIC simply corresponds to reversing the average force (i.e., $x \rightarrow-x$ ) in Sinai's model. As will be discussed below, the deviation from criticality parameter $2 \delta$ in the RTFIC corresponds to the parameter $\mu / T$ in Sinai's model (see [13,14]) which controls the long time properties and the various phases and is defined for the original model with unit length bonds by

$$
\left\langle\exp \left(-\mu f_{n} / T\right)\right\rangle=1
$$

Zero drift corresponds to criticality in the RTFIC [21], while the biased phase with zero velocity [13,14] corresponds to the Griffiths phase of the RTFIC [21] as will be discussed below. Note however that the physical quantities of interest in the two models can be different.

\section{Effective dynamics and validity of the method}

Throughout the paper, we define the "effective dynamics" as the dynamics which consists in putting the particle at time $t$ at the bottom of the renormalized valley at scale $\Gamma=T \ln \left(t / t_{0}\right)$ which contains the starting point at $t=0$ (see Fig. 2). Thus in the effective dynamics the particle does not move unless one of the bonds which

are the sides of the renormalized valley to which it belongs is decimated, in which case it jumps to the bottom of the new renormalized bond as in Fig. 2. Here, $t_{0}$ is a 
non-universal microscopic time scale, which throughout the paper we set to unity by appropriate redefinition of time units; we can then use interchangably $\Gamma$ and $T \ln t$.

Symmetric case

This effective dynamics is an approximation of the true dynamics. But within the RG approach it can be seen that this approximation becomes better and better as $\Gamma=T \ln t$ increases since the distribution of barriers $P_{\Gamma}(F)$ becomes broader and broader, as is detailed below. Thus the renormalized landscape consists entirely of deep valleys separated by high barriers and with high probability the particle will be near the bottom of the valley in which it began. Upon rescaling of space as $X=x / \Gamma^{2}$ the effective dynamics of the diffusion front becomes exact as $\Gamma$ tends to $\infty$ as was proven in ref. [11]. Indeed, the probability that the walker is close -in a precise sense that we discuss later - to its position given by the effective dynamics, approaches one at long times. This stronger result has also been rigorously established. [11, 12, 26].

There is clearly a source of error in the approximation of the true dynamics by the effective dynamics when two neighboring bonds have barriers $F$ 's that are within order $T$ of each other. However, the error introduced by assigning a particle to one of two almost-equal-depth neighboring valleys rather than splitting its distribution between the two valleys will occur more and more rarely at long scales. Furthermore, any such error is wiped out by a later decimation which eliminates the two valleys in favor of a deeper valley. These errors thus lead only to subdominant contributions to the quantities that we will compute - with the exception of tails of certain distributions which are dominated by rare configurations of the lansdscape. These subdominant corrections can themselves be estimated via the RG. For instance the rescaled mean square thermal width of a packet $\frac{1}{\Gamma^{4}} \overline{\left\langle x(t)^{2}\right\rangle-\langle x(t)\rangle^{2}}$ (with overbars denoting averaging over landscapes) tends to 0 for large $\Gamma$ which is its value in the effective dynamics, but has $1 / \Gamma$ corrections coming from barrier degeneracies, estimated in Section (VIH).

Strong differences between the real dynamics and the effective dynamics can appear in some quantities, such as the persistence properties studied in Sections (IV], V). These quantities are usually in some way nonlocal in time and depend on the behavior of the system over time. Even in these cases though, as we show in Section $(\mathbb{I V}, \nabla)$, it is possible to compute some of these quantities by a proper interpretation and examination of the $\mathrm{RG}$ procedure.

\section{Biased case}

In the biased case with a bias $\delta>0$ one finds within the RG that the distribution of barriers against the drift is no longer infinitely broad. However if $\delta$ is small the barriers remain large enough so that the RSRG remains a good approximation. Again, this approximation remains exact, in the same sense as above, in the appropriate scaling limit fixed $\delta \Gamma$ and $x / \Gamma^{2}$ (corresponding to the critical region of the RTFIC). For a fixed $\delta$ one expects that the thermal packet is spread over several deep wells, but when $\delta \rightarrow 0$ the contributions of these few additional wells becomes subdominant.

To conclude this Section, we stress that despite its approximate character, our RSRG method allows us to obtain exact results for many quantities both for the symmetric and the weakly biased Sinai model. 


\section{3. $R G$ with one boundary: reflecting or absorbing}

We now consider the problem of diffusion in a semi-infinite one-dimensional medium defined as $x>0$. In practice there are two main types of boundary conditions for the diffusing particle: (i) reflecting (the current at the boundary is zero) and (ii) absorbing (the probability is zero at the boundary). We show in this section how both boundary conditions can be treated by adding to the bulk RSRG specific rules near the boundary, which we call boundary RSRG.

Let us start with the zero bias case and a reflecting boundary. This condition can be represented by placing a barrier with infinite potential $U_{0}=+\infty$ at $x=0$ with $U_{1}$ finite, as is illustrated in Fig. 3.
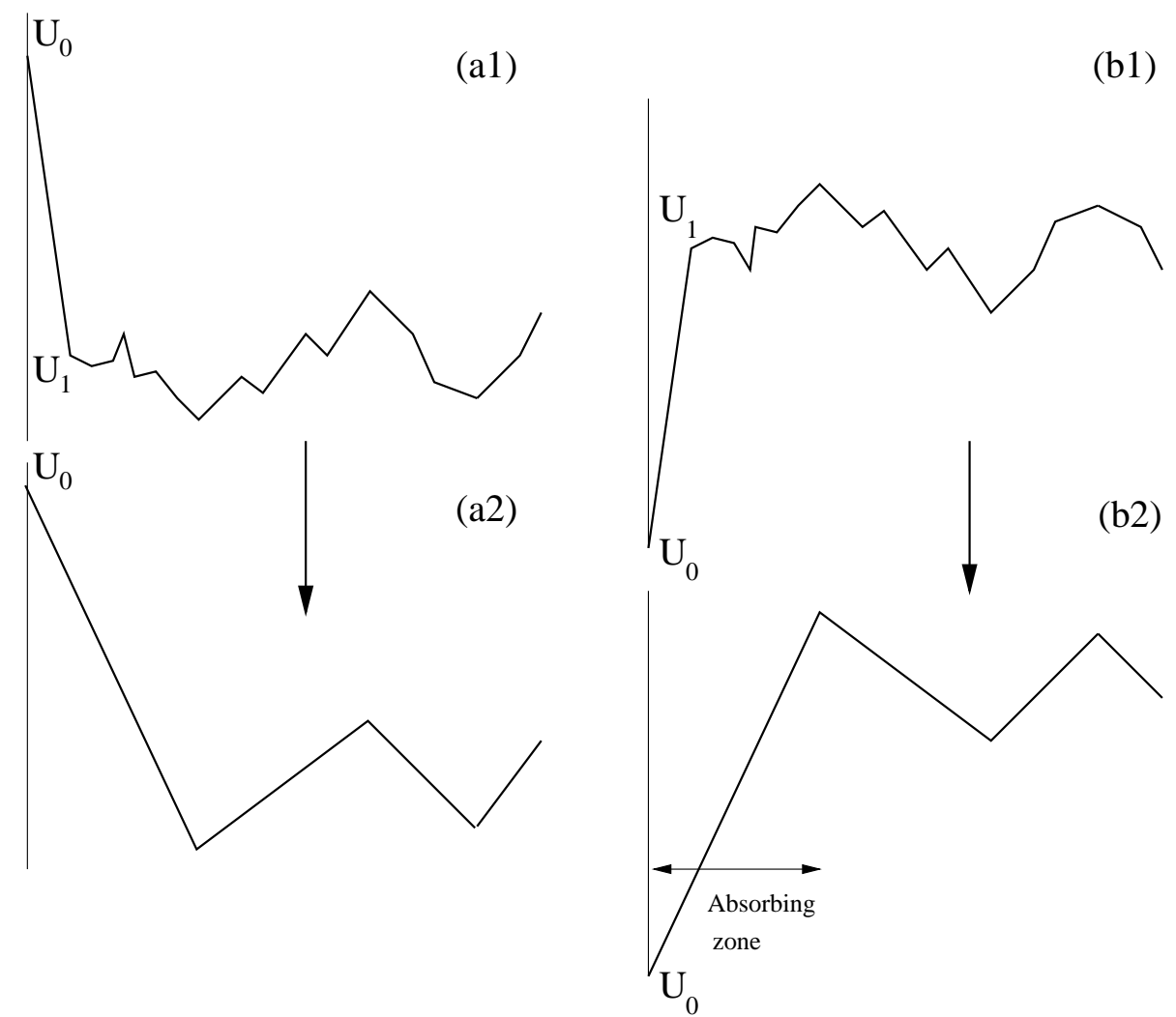

FIG. 3. Illustration of the RG in presence of a boundary. (a1) Reflecting boundary conditions: the boundary at site $x=0$ can be represented by setting $U_{0}=+\infty$. (a2) Renormalized landscape. (b1) Absorbing boundary conditions: the boundary at site $x=0$ can be represented by setting $U_{0}=-\infty$ (b2) Renormalized landscape, with the absorbing zone (see text).

When grouping bonds with the same sign as in the previous section, the first bond will always be descending with an infinite barrier $F_{1}=+\infty$ and a length $l_{1}$. The decimation of the landscape then proceeds as in the bulk case except that now the first bond is never decimated and when the second bond gets decimated (at $\Gamma=F_{2}$ ) it simply increases the length of the first bond $l_{1}^{\prime}=l_{1}+l_{2}+l_{3}$. One can easily see that starting from a landscape where bonds are statistically uncorrelated - with a distribution $E_{\Gamma}(l)$ for the first bond and $P_{\Gamma}(F, l)$ for all the other bonds - they remain so under the boundary RSRG. Upon increase in $\Gamma$, the bulk distribution $P$ obeys the same RG equation (6) while $E$ satisfies: 


$$
\partial_{\Gamma} E_{\Gamma}(l)=-P_{\Gamma}(\Gamma) E_{\Gamma}(l)+E_{\Gamma}(.) *_{l} P_{\Gamma}(\Gamma, .) *_{l} P_{\Gamma}(.)
$$

The case of an absorbing boundary can be treated in the same way since it amounts to setting the potential of the site $x=0$ to $U_{0}=-\infty$. This is illustrated in Fig. 3. Thus the first bond will always be ascending with an infinite barrier $F_{1}=+\infty$ and a length $l_{1}$ (and thus cannot be decimated). The rules are thus the same as above with the same RG equation (9) for the distribution $E_{\Gamma}(l)$ of the length of the first bond. The interpretation is however different: the first bond represent an "absorbing zone" such that any particle starting from a point within this zone will be absorbed by the boundary before time $\Gamma=T \ln t$, while the particles starting outside this zone are still "alive" (and outside this zone) at $\Gamma=T \ln t$ (with probability asymptotically close to one).

We note at this stage that this equation coincides with the RG equation for the endpoint magnetization in the RTFIC; i.e., with the first exchange being $J_{0}=0$. Conversely, a reflecting boundary corresponds to the first transverse field being $h_{1}=0$. The equivalence is reversed on the other end of the chain [21].

In the case of a bias, the probability distribution of the first renormalized bond $E_{\Gamma}^{ \pm}(l)$ (+ when the bond is along the bias, and - when it is against) satisfies:

$$
\partial_{\Gamma} E_{\Gamma}^{ \pm}(l)=P_{\Gamma}^{\mp}(0, .) *_{l} E_{\Gamma}^{ \pm}(.) *_{l} \int_{0}^{\infty} d \zeta^{\prime} P_{\Gamma}^{ \pm}\left(\zeta^{\prime}, .\right)-E_{\Gamma}^{ \pm}(l) \int_{0}^{\infty} d l^{\prime} P_{\Gamma}^{\mp}\left(0, l^{\prime}\right)
$$

which generalizes equation (9) of the zero bias case.

\section{General analysis of the RSRG equations}

In this Section we recall some results from references [20,21, 18, 22] which will be used extensively in this paper, about the large $\Gamma$ behavior of the solutions of the RSRG equations (6, 10) and discuss them in the context of the Sinai model.

\section{Symmetric model}

We start with the symmetric Sinai model (zero bias, self-dual) and thus the RG of a single distribution $P^{+}=P^{-}$. One first defines the large-scale variance $\sigma$ of the potential as

$$
\overline{\left(U_{i}-U_{j}\right)^{2}} \approx 2 \sigma\left|l_{i-j}\right|
$$

with $l_{i-j}$ the distance from $i$ to $j$. Since both $l_{i-j}$ and $U_{i}-U_{j}$ are preserved by the $\mathrm{RG}, \sigma$ is also preserved and determined by the initial model as $2 \sigma=\int d f f^{2} Q(f)$. In the remainder of the paper we will absorb $\sigma$ in $l$ and simply study the case $\sigma=1$. The units of length are then $1 / \sigma$. To obtain the full results one must change $l \rightarrow \sigma l$ (and $d l \rightarrow \sigma d l$ ) in the following formulae. The rescaled probability $P_{\Gamma}(\eta, \lambda) \equiv \Gamma^{3} P_{\Gamma}\left(\eta \Gamma, \lambda \Gamma^{2}\right)$ in terms of the rescaled variables

$$
\eta=\zeta / \Gamma, \quad \lambda=l / \Gamma^{2}
$$

satisfies, when Laplace transformed in $\lambda \rightarrow p$ : 


$$
\left(\Gamma \partial_{\Gamma}-(1+\eta) \partial_{\eta}+2 p \partial_{p}-1\right) P_{\Gamma}(\eta, p)=P_{\Gamma}(0, p) P_{\Gamma}(., p) *_{\eta} P_{\Gamma}(., p)
$$

The fixed point solution [21] is found to be

$$
\begin{aligned}
& \tilde{P}(\eta, p)=a(p) e^{-\eta b(p)} \\
& \text { with } a(p)=\frac{\sqrt{p}}{\sinh (\sqrt{p})} \quad b(p)=\sqrt{p} \operatorname{coth}(\sqrt{p}) .
\end{aligned}
$$

Thus, taking $p=0$, one finds the physically natural result that, due to the occurrence of long regions which are predominantly up or predominantly down, the coarsegrained probability distribution of barriers in Sinai's model is exponential

$$
P_{\Gamma}(F) \simeq \frac{\theta(F-\Gamma)}{\Gamma} e^{-\frac{(F-\Gamma)}{\Gamma}}
$$

with a width which grows as $\langle F\rangle \sim \Gamma \sim T \ln t$. The total number of bonds satisfies (7) and thus decays asymptotically as $n_{\Gamma} \sim \Gamma^{-2}$ and the average bond length (8) grows as $\sim \Gamma^{2}$. Since $\Gamma \sim \ln t$ one recovers Sinai's scaling [11]

$$
x \sim \ln ^{2} t .
$$

In the following we will need the explicit form of the distribution $P(\lambda)=$ $\int_{0}^{\infty} d \eta P(\eta, \lambda)$

$$
\begin{aligned}
& P(\lambda)=L T_{p \rightarrow \lambda}^{-1}\left(\frac{a(p)}{b(p)}\right)=L T_{p \rightarrow \lambda}^{-1}\left(\frac{1}{\cosh (\sqrt{p})}\right) \\
& =\sum_{n=-\infty}^{\infty}\left(n+\frac{1}{2}\right) \pi(-1)^{n} e^{-\pi^{2} \lambda\left(n+\frac{1}{2}\right)^{2}}=\frac{1}{\sqrt{\pi} \lambda^{3 / 2}} \sum_{m=-\infty}^{\infty}(-1)^{m}\left(m+\frac{1}{2}\right) e^{-\frac{1}{\lambda}\left(m+\frac{1}{2}\right)^{2}}
\end{aligned}
$$

It was shown in [21] that the convergence towards the fixed point solution $P^{*}(\eta)=e^{-\eta}$ on the critical manifold (i.e., symmetric perturbations) is like $\frac{1}{\Gamma}$ with

eigenvector $P_{\Gamma}^{(1)}(\eta)=(\eta-1) e^{-\eta}$ - corresponding simply to a shift in $\Gamma$-plus other parts that decay exponentially in $\Gamma$ and depend on tails in the initial distributions.

\section{Biased model}

In the case of the biased model one must follow the descending bond distribution $P_{+}$and the ascending bond distribution $P_{-}$which are different. Contrary to the previous section, it is more convenient in this case to use the unrescaled distributions and variables. In terms of the Laplace transforms $P_{\Gamma}^{ \pm}(p, l)=\int_{0}^{+\infty} d l e^{-p l} P_{\Gamma}^{ \pm}(\zeta, l)$ the equation (6) reads:

$$
\left(\partial_{\Gamma}-\partial_{\zeta}\right) P^{ \pm}(\zeta, p)=P^{\mp}(0, p) P^{ \pm}(., p) *_{\zeta} P^{ \pm}(., p)+\left(P^{ \pm}(0,0)-P^{\mp}(0,0)\right) P^{ \pm}(\zeta, p)
$$

As was shown in [21], for large $\Gamma$ the distributions $P_{ \pm}$take the following form, in the scaling regime of small $\delta$ and small $p$ with $\delta \Gamma$ fixed and $p \Gamma^{2}$ fixed: 


$$
\begin{aligned}
P_{\Gamma}^{ \pm}(\zeta, p) & =U_{\Gamma}^{ \pm}(p) e^{-\zeta u_{\Gamma}^{ \pm}(p)} \\
u_{\Gamma}^{ \pm}(p) & =\sqrt{p+\delta^{2}} \operatorname{coth}\left[\Gamma \sqrt{p+\delta^{2}}\right] \mp \delta \\
U_{\Gamma}^{ \pm}(p) & =\frac{\sqrt{p+\delta^{2}}}{\sinh \left[\Gamma \sqrt{p+\delta^{2}}\right]} e^{\mp \delta \Gamma}
\end{aligned}
$$

[Note that here we use $U$ rather than the $\Upsilon$ of [21].] We will also use the evolution equations obtained by substituting (22) in the RG equation (16):

$$
\begin{aligned}
& \partial_{\Gamma} u_{\Gamma}^{ \pm}(p)=-U_{\Gamma}^{+}(p) U_{\Gamma}^{-}(p) \\
& \partial_{\Gamma} U_{\Gamma}^{ \pm}(p)=-u_{\Gamma}^{\mp}(p) U_{\Gamma}^{ \pm}(p)
\end{aligned}
$$

The distributions of barriers alone are:

$$
\begin{array}{r}
P^{-}(\zeta)=\frac{2 \delta}{1-e^{-2 \Gamma \delta}} \exp \left(-\zeta \frac{2 \delta}{1-e^{-2 \Gamma \delta}}\right) \\
P^{+}(\zeta)=\frac{2 \delta}{e^{2 \Gamma \delta}-1} \exp \left(-\zeta \frac{2 \delta}{e^{2 \Gamma \delta}-1}\right)
\end{array}
$$

and the average lengths of the $( \pm)$-bonds are respectively given by:

$$
\begin{aligned}
& \bar{l}^{+}=\frac{1}{2 \delta^{2}}\left(e^{\delta \Gamma} \sinh (\delta \Gamma)-\delta \Gamma\right) \\
& \bar{l}^{-}=\frac{1}{2 \delta^{2}}\left(\delta \Gamma-e^{-\delta \Gamma} \sinh (\delta \Gamma)\right)
\end{aligned}
$$

When $\delta \rightarrow 0$ one has $\bar{l}^{ \pm} \rightarrow \frac{1}{2} \Gamma^{2}$. and thus the total average length of a valley is:

$$
\bar{l}_{\Gamma}=\bar{l}^{+}+\bar{l}^{-}=\left(\frac{\sinh (\Gamma \delta)}{\delta}\right)^{2} \sim n_{\Gamma}^{-1}
$$

where $n_{\Gamma}$ is the total number of bonds.

The convergence towards the solution (22) has been discussed in [21]. The above solution (22) thus depends on an "integration constant" $2 \delta=u_{\Gamma}^{-}(p=0)-u_{\Gamma}^{+}(p=0)$ which is determined by the initial condition, and is proportional to the drift. In [21] it was identified for small $\delta$ as the ratio of the mean to the variance of the original distribution $Q(f)$ of the initial independent bonds of unit length 1; i.e. before grouping the bonds together:

$$
\delta=\frac{\bar{f}}{\overline{f^{2}}-\bar{f}^{2}}
$$

It is useful to introduce a parameter $\mu$ defined by the unique non zero solution of the equation

$$
\overline{e^{-\mu f / T}}=\int_{-\infty}^{+\infty} d f Q(f) e^{-\mu f / T}=1
$$

This parameter $\mu$ has been introduced previously in [25] and is known to determine exactly the various phases of the dynamics of the Sinai model with a bias $\left(x \sim t^{\mu}\right.$ 
for $\mu>1, x \sim t$ for $\mu>1$ ). Indeed it is also known in random walk theory 443 to control the probability of large excursions against the bias. We now show that we can interpret these properties within the RG as associated with the exact decimation of the landscape (6).

The distributions $P_{0}^{ \pm}(F)$ of the barriers $F>0$ of the zig-zag landscape (see Fig. 2) obtained by grouping together the consecutive ascending or descending bonds of the original discrete model, are related to the original $Q(f)$ distribution through

$$
\int_{0}^{\infty} d F e^{-s F} P_{0}^{ \pm}(F)=\frac{Q^{\mp}(0)}{Q^{ \pm}(0)} \frac{Q^{ \pm}(s)}{1-Q^{ \pm}(s)}
$$

where $Q^{+}(s)=\int_{0}^{+\infty} d f e^{-s f} Q(f)$ and $Q^{-}(s)=\int_{-\infty}^{0} d f e^{s f} Q(f)$. The difference of potential $\left(F_{+}-F_{-}\right)$of the boundaries of a valley of the initial zig-zag potential has Laplace transform

$$
\int_{0}^{\infty} d F_{+} e^{-s F_{+}} P_{0}^{+}\left(F_{+}\right) \int_{0}^{\infty} d F_{-} e^{s F_{-}} P_{0}^{-}\left(F_{-}\right)=\frac{Q^{+}(s) Q^{-}(-s)}{1-Q(s)+Q^{+}(s) Q^{-}(-s)}
$$

where $Q(s)=Q^{+}(s)+Q^{-}(-s)=\int_{-\infty}^{+\infty} d f Q(f) e^{-s f}$. The definition of $\mu$, Eq. (28), for the original model is thus equivalent for the initial zig-zag landscape to defining $\mu$ as the unique non-zero solution of the equation

$$
\left\langle e^{-\mu F_{+} / T}\right\rangle_{P_{0}^{+}}\left\langle e^{\mu F_{-} / T}\right\rangle_{P_{0}^{-}}=1
$$

But since the renormalized valleys at scale $\Gamma$ are constructed from the valleys of the initial zig-zag potential and are statistically uncorrelated, this implies that for the probability distributions $P_{\Gamma}^{ \pm}(F)$ of the renormalized barriers at any scale $\Gamma$,

$$
\left\langle e^{-\mu F_{+} / T}\right\rangle_{P_{\Gamma}^{+}}\left\langle e^{\mu F_{-} / T}\right\rangle_{P_{\Gamma}^{-}} \equiv \int_{\Gamma}^{\infty} d F_{+} e^{-\mu F_{+} / T} P_{\Gamma}^{+}\left(F_{+}\right) \int_{\Gamma}^{\infty} d F_{-} e^{\mu F_{-} / T} P_{\Gamma}^{-}\left(F_{-}\right)=1
$$

Using the explicit solutions (22) for the distributions of barriers, we obtain in terms of $u_{\Gamma}^{ \pm}=u_{\Gamma}^{ \pm}(p=0)$ the following equation for $\mu$

$$
1=\left(\frac{u_{\Gamma}^{+}}{u_{\Gamma}^{+}+\frac{\mu}{T}}\right)\left(\frac{u_{\Gamma}^{-}}{u_{\Gamma}^{-}-\frac{\mu}{T}}\right)=\frac{1}{1+\frac{\mu}{T u_{\Gamma}^{+} u_{\Gamma}^{-}}\left(\left(u_{\Gamma}^{-}-u_{\Gamma}^{+}\right)-\frac{\mu}{T}\right)}
$$

and we thus obtain that the parameter $2 \delta=u_{\Gamma}^{-}-u_{\Gamma}^{+}$parametrizing the RG solutions (22) indeed corresponds to the parameter $\mu / T$. Note, however, that the expression (27) is only valid for small $\delta$.

Thus even away from small $\mu$, the RSRG allows one to obtain exact information on the structure of the landscape, in particular the behavior of the probability of large barriers impeding the drift. For large $\Gamma$ we have, from (22) that (for positive $\delta) P^{-}(\zeta) \approx 2 \delta e^{-2 \delta \zeta}$ implying that the probability of a large barrier, $F$, is $\sim \exp (-\mu F / T)$. As we will see shortly, this controls the anomalous drift exponent $\mu$. 


\section{Boundary fixed point solutions}

The RG equation for the distribution of lengths of the boundary bond $E^{\mp}(l)$ defined in section ([IB3) was given in (10). In the Laplace variable with respect to length the $\mathrm{RG}$ equation (10) reads:

$$
\partial_{\Gamma} E_{\Gamma}^{ \pm}(p)=E_{\Gamma}^{ \pm}(p)\left(P_{\Gamma}^{\mp}(0, p) P_{\Gamma}^{ \pm}(p)-P_{\Gamma}^{\mp}(0, p=0)\right)
$$

For large $\Gamma$ using the properties of the fixed point solution (22) for $P^{ \pm}$and the properties (20) of the functions $U$ and $u$, this can be rewritten as:

$$
\partial_{\Gamma} \ln E_{\Gamma}^{ \pm}(p)=\frac{U_{\Gamma}^{+}(p) U_{\Gamma}^{-}(p)}{u_{\Gamma}^{ \pm}(p)}-u_{\Gamma}^{\mp}(0)=\partial_{\Gamma}\left(\ln U_{\Gamma}^{ \pm}(0)-\ln u_{\Gamma}^{ \pm}(p)\right)
$$

Finally we find:

$$
E_{\Gamma}^{ \pm}(p)=\frac{u_{\Gamma}^{ \pm}(0)}{u_{\Gamma}^{ \pm}(p)}=\frac{\delta e^{\mp \delta \Gamma}}{\sinh (\delta \Gamma)\left(\sqrt{p+\delta^{2}} \operatorname{coth}\left[\Gamma \sqrt{p+\delta^{2}}\right] \mp \delta\right)}
$$

In the symmetric case $\delta=0$ we get

$$
E_{\Gamma}(p)=\frac{\sinh \Gamma \sqrt{p}}{\Gamma \sqrt{p} \cosh \Gamma \sqrt{p}}
$$

whose inverse Laplace transform reads in the rescaled variable

$$
\lambda \equiv \frac{l}{\Gamma^{2}}
$$

is

$$
E(\lambda)=\sum_{n=-\infty}^{+\infty} e^{-\lambda \pi^{2}\left(n+\frac{1}{2}\right)^{2}}=\frac{1}{\sqrt{\pi \lambda}} \sum_{m=-\infty}^{+\infty}(-1)^{m} e^{-\frac{m^{2}}{\lambda}}
$$

\section{SINGLE TIME DIFFUSION PROPERTIES IN SINAI MODEL}

In this Section we study the diffusion front using the effective dynamics introduced in Section (IIB2).

\section{A. Single time diffusion front for the symmetric model}

We now consider the (single time) diffusion front, i.e., the probability $\operatorname{Prob}\left(x, t \mid x_{0}, 0\right)$ that a particle starting at $x_{0}$ at $t=0$ be located at $x$ at time $t$, for the symmetric, zero bias case. At large time $t$ the effective (renormalized) dynamics corresponds to moving the particle from its starting point $x_{0}$ to the lower-potential end of the renormalized bond at scale $\Gamma=T \ln t$ that contains $x_{0}$. This is illustrated in Fig 2. In a single environment $\operatorname{Prob}\left(x, t \mid x_{0}, 0\right)$ is thus localized near the bottom 
of the bond-i.e., the bottom of a valley - and the rescaled position $x / \ln ^{2} t$ has a delta function shape at large time.

One can compute averages over environments, or equivalently over initial conditions $x_{0}$ (with a spatially uniform measure) in a single environment. The average diffusion front $\overline{\operatorname{Prob}\left(x_{0}+x, t \mid x_{0}, 0\right)}$ is obtained as follows. The probability that a given bond has length $l$ is $P_{\Gamma}(l)=\int d \eta P_{\Gamma}(\eta, l)$ and the probability density that $x_{0}$ belongs to a renormalized bond of length $l$ at scale $\Gamma$ is $l P_{\Gamma}(l) / \int_{l} l P_{\Gamma}(l)$. Taking into account that the distance $|x|$ between the starting point $x_{0}$ and the bottom of the bond is uniformly distributed on $[0, l]$, one finds after averaging over $l$ :

$$
\overline{\operatorname{Prob}(x, t \mid 0,0)}=\frac{1}{2 \int_{l} l P_{\Gamma}(l)} \int_{|x|}^{\infty} d l P_{\Gamma}(l)
$$

Using the fixed point solution (11, 14) with $\Gamma=T \ln t$, we find that the diffusion front takes the scaling form:

$$
\begin{array}{r}
\overline{\operatorname{Prob}(x, t \mid 0,0)}=\frac{\sigma}{T^{2} \ln ^{2} t} q\left(\frac{\sigma x}{T^{2} \ln ^{2} t}\right) \\
\text { with } \quad q(X)=\frac{4}{\pi} \sum_{n=0}^{\infty} \frac{(-1)^{n}}{2 n+1} e^{-\frac{1}{4} \pi^{2}|X|(2 n+1)^{2}}
\end{array}
$$

where we have reinserted $\sigma$. This coincides with the Kesten-Golosov rigorous result [12,27] for a Brownian potential, as it should [11] since our method gives exact results for properties of the rescaled walks $x(t) / \ln ^{2} t$.

\section{B. Single time diffusion front for the biased model}

The case of a global bias $\langle f\rangle_{Q}>0$ is described by the RG equations (6) with $P^{+} \neq P^{-}$. The fixed point (22) was analyzed in the previous section. It shows that at large scales $\Gamma$ the barriers impeding the drift have an exponential distribution that does not continue to broaden:

$$
P_{\Gamma}^{-}(F) \sim 2 \delta e^{-2 \delta(F-\Gamma)} \theta(F-\Gamma)
$$

On the other hand, the bonds along the drift become very long with large barriers:

$$
P_{\Gamma}^{+}(F) \sim \frac{1}{F_{\Gamma}} e^{-\frac{(F-\Gamma)}{F_{\Gamma}}} \theta(F-\Gamma)
$$

where $F_{\Gamma} \sim \frac{1}{2 \delta} e^{2 \delta \Gamma} \sim \frac{1}{2 \delta} t^{\mu}$. Asymptotically in the RG only barriers impeding the drift are decimated, since the barriers to go against the bias are very large. The distribution Eq. (41) is then simply that of potential drops between the impeding barriers. One thus recovers the physical picture [14] that Sinai's biased diffusion renormalizes onto a directed model with traps (ascending bonds) of release times $\tau$ with distribution $\rho(\tau) \sim \tau^{-(1+\mu)}$. The average length $\bar{l}_{\Gamma}^{+}$of the descending bond distribution Eq. (24) yields the anomalous diffusion scaling $x \sim t^{\mu}$.

We now compute the average diffusion front $\overline{\operatorname{Prob}(x, t \mid 0,0)}$ in the case of a small average potential drop per unit length $2 \delta>0$. The argument is as in the symmetric case, except that one must distinguish $x>0$ from $x<0$, which correspond 
respectively to the starting point being on a descending, $\left(P^{+}\right)$or ascending $\left(P^{-}\right)$ renormalized bond at scale $\Gamma$. One thus uses the formula:

$$
\overline{\operatorname{Prob}(x, t \mid 0,0)}=\frac{1}{\bar{l}_{\Gamma}}\left[\theta(x) \int_{x}^{+\infty} d l P_{\Gamma}^{+}(l)+\theta(-x) \int_{-x}^{+\infty} d l P_{\Gamma}^{-}(l)\right]
$$

This yields, in the scaling limit where $\Gamma$ is large while $\lambda=l / \Gamma^{2}$ and $\gamma \equiv \Gamma \delta$ are both fixed but arbitrary, the generalization of $\mathrm{Eq}(39)$ :

$$
\overline{\operatorname{Prob}(x, t \mid 0,0)}=\frac{\sigma}{T^{2}(\ln t)^{2}} q\left(X=\frac{\sigma x}{T^{2}(\ln t)^{2}}, \gamma=T \delta \ln t\right)
$$

with

$$
\begin{aligned}
& q(X, \gamma)=\left(\frac{\gamma}{\sinh \gamma}\right)^{2}\left[\theta(X) L T_{s \rightarrow X}^{-1} \frac{1}{s}\left(1-\frac{\kappa e^{-\gamma}}{\kappa \cosh \kappa-\gamma \sinh \kappa}\right)\right. \\
& \left.+\theta(-X) L T_{s \rightarrow-X}^{-1} \frac{1}{s}\left(1-\frac{\kappa e^{\gamma}}{\kappa \cosh \kappa+\gamma \sinh \kappa}\right)\right],
\end{aligned}
$$

with $\kappa \equiv \sqrt{s+\gamma^{2}}$. From this expression we can compute the moments. One finds:

$$
\begin{aligned}
\overline{\langle x(t)\rangle}= & \frac{1}{8 \delta^{2} \sinh [\gamma]^{2}}(\sinh [4 \gamma]-6 \gamma \cosh [2 \gamma]+\sinh [2 \gamma]) \\
\overline{\left\langle x(t)^{2}\right\rangle}= & \frac{1}{16 \delta^{4} \sinh [\gamma]^{2}}\left(\cosh [6 \gamma]-10 \gamma \sinh [4 \gamma]+3 \cosh [4 \gamma]+18 \gamma^{2} \cosh [2 \gamma]\right. \\
& \left.-12 \gamma \sinh [2 \gamma]+\cosh [2 \gamma]+2 \gamma^{2}-5\right)
\end{aligned}
$$

Note that $\overline{\langle x(t)\rangle} \approx \frac{3}{5} \delta \Gamma^{3}$ for small $\gamma=\delta \Gamma$, a form implied by scaling and analyticity in $\delta$.

One can also perform the Laplace inversion. For $\gamma<1$ let us introduce the roots $\alpha_{n}^{ \pm}(\gamma)(n=0,1, \ldots)$ of the equation:

$$
\alpha_{n}^{ \pm}(\gamma) \operatorname{cotan}\left(\alpha_{n}^{ \pm}(\gamma)\right)= \pm \gamma \quad \text { with } \quad n \pi<\alpha_{n}^{ \pm}(\gamma)<(n+1) \pi
$$

For $\gamma>1$, the root $\alpha_{0}^{+}(\gamma)$ does not exist, but is replaced by the positive root $\tilde{\alpha}_{0}^{+}(\gamma)$ of the equation $\tilde{\alpha}_{0}^{+}(\gamma) \operatorname{coth}\left(\tilde{\alpha}_{0}^{+}(\gamma)\right)=\gamma$. In terms of these roots, the Laplace inversion gives

$$
q(X, \gamma)=\theta(X) \sum_{n=0}^{\infty} c_{n}^{+}(\gamma) e^{-X s_{n}^{+}(\gamma)}+\theta(-X) \sum_{n=0}^{\infty} c_{n}^{-}(\gamma) e^{-|X| s_{n}^{-}(\gamma)}
$$

where

$$
\begin{aligned}
& s_{n}^{ \pm}(\gamma)=\gamma^{2}+\left(\alpha_{n}^{ \pm}(\gamma)\right)^{2} \\
& c_{n}^{ \pm}(\gamma)=\left(\frac{\gamma}{\sinh \gamma}\right)^{2} \frac{2(-1)^{n+1}\left(\alpha_{n}^{ \pm}(\gamma)\right)^{2} e^{\mp \gamma}}{\sqrt{\gamma^{2}+\left(\alpha_{n}^{ \pm}(\gamma)\right)^{2}}\left(\gamma^{2}+\left(\alpha_{n}^{ \pm}(\gamma)\right)^{2} \mp \gamma\right)}
\end{aligned}
$$

except for the term $n=0$ in the domain $X>0$ and $\gamma>1$ for which

$$
\begin{aligned}
& s_{0}^{+}(\gamma>1)=\gamma^{2}-\left(\tilde{\alpha}_{0}^{+}(\gamma)\right)^{2} \\
& c_{0}^{+}(\gamma>1)=\left(\frac{\gamma}{\sinh \gamma}\right)^{2} \frac{2\left(\tilde{\alpha}_{0}^{+}(\gamma)\right)^{2} e^{-\gamma}}{\sqrt{\gamma^{2}-\left(\tilde{\alpha}_{0}^{+}(\gamma)\right)^{2}}\left(\gamma+\left(\tilde{\alpha}_{0}^{+}(\gamma)\right)^{2}-\gamma^{2}\right)}
\end{aligned}
$$


Note that $s_{0}^{+}(\gamma)$ is an analytic function of $\gamma$ despite its definition by two domains. In the limit of small $\gamma$, we recover of course the symmetric case Eq. (39) using $\alpha_{n}^{ \pm}(\gamma \rightarrow 0)=(1+2 n) \frac{\pi}{2}$. For large $\gamma \gg 1$, i.e., $T \ln t \gg 1 / \delta$, we have $\tilde{\alpha}_{0}^{+}(\gamma) \simeq$ $\gamma\left(1-2 e^{-2 \gamma}+\ldots\right)$ and thus

$$
\begin{aligned}
& s_{0}^{+}(\gamma \gg 1) \simeq 4 \gamma^{2} e^{-2 \gamma} \\
& c_{0}^{+}(\gamma \gg 1) \simeq 4 \gamma^{2} e^{-2 \gamma}
\end{aligned}
$$

whereas all other coefficients in exponentials are much bigger since $s_{n}^{ \pm}(\gamma)>\gamma^{2}$. In the regime $\gamma \gg 1$, the distribution is thus heavily concentrated to the right of the origin and reduces to the simple exponential:

$$
\overline{\operatorname{Prob}(x, t \mid 0,0)} \approx \theta(x) \exp [-x / \overline{x(t)}] \overline{x(t)}
$$

with the mean displacement

$$
\overline{x(t)} \approx t^{2 \delta T} /\left(4 \delta^{2}\right)
$$

We can now compare with known results [11,14: for fixed $0<\mu<1$ the variable $\tilde{x}=x / t^{\mu}$ is distributed with a half-sided Levy probability density $L_{\mu}\left(\tilde{x}^{-1 / \mu}\right) d \tilde{x}^{-1 / \mu}$ where $L_{\mu}(z)=L T_{s \rightarrow z}^{-1} e^{-C_{\mu} s^{\mu}}$. Our asymptotic result (56) reproduces correctly the the small $\mu$ limit of this Levy front [11,14] with the correct prefactor $C_{\mu}$.

\section{MOTION OF THERMAL AVERAGES: RETURNS TO THE ORIGIN AND JUMPS}

We now study "recurrence" properties of the Sinai model. One must carefully distinguish between the effective dynamics (i.e., the walker jumping between valley bottoms) and the real dynamics. In this section we concentrate on the effective dynamics. This amounts, as we will see, to studying the fine structure of the motion of the (thermal) packet. Asking similar question for a single particule requires a study in the presence of absorbing walls and will be discussed in the next section. We will also study the zero crossings of the "running average"

$$
\Xi(t) \equiv \frac{1}{t} \int_{0}^{t} x(\tau) d \tau
$$

which is an approximation to the thermal average.

While in a single "run" in a given environment the walker typically crosses its starting point many times while trapped in a valley, averaging over many runs in the same environment yields a $\langle x(t)\rangle$ which crosses $x_{0}$ exactly once each time the bond on which $x_{0}$ lies is decimated, since this causes its valley bottom to cross $x_{0}$.

We will first ask what is the fraction $M_{k}(t)$ of starting points, $x_{0}$, for which the thermally averaged position $\left\langle x(t) \mid x(0)=x_{0}\right\rangle$ has crossed $x_{0}$ exactly $k$ times up to time $t$. Since the effective dynamics consists in putting the particule at the lowest point of the decimated bond, the origin and the particule remain in the same bond at all times. The probability of crossing the origin - i.e., the starting point - between 0 and $t$ exactly $k$ times is thus the fraction of sites which belong to bonds which have changed orientation exactly $k$ times between 0 and $t$. In particular, the probability of no return to the origin is $M_{0}(t)$ and is equal to the probability that the bond containing the origin has never been decimated. 


\section{A. Number of returns to the origin: symmetric case}

To compute $M_{k}(t)$ we use two equivalent methods, which we both describe as they will be useful in the remainder of the paper.

First method: Let $N_{\Gamma}(k, \eta)$ be the probability that the bond containing the origin has a rescaled barrier $\eta=(F-\Gamma) / \Gamma$ at $\Gamma$ and has switched its orientation $k$ times up to scale $\Gamma$. It is normalized as $\sum_{k=0}^{+\infty} \int_{0}^{+\infty} d \eta N_{\Gamma}(k, \eta)=1$ and it satisfies :

$$
\begin{aligned}
& \left(\Gamma \partial_{\Gamma}-(1+\eta) \partial_{\eta}-1\right) N_{\Gamma}(k, \eta)=2 P_{\Gamma}(0) N_{\Gamma}(k, .) *_{\eta} P_{\Gamma}(.) \\
& -2 P_{\Gamma}(0) N_{\Gamma}(k, \eta)+N_{\Gamma}(k-1,0) P_{\Gamma}(.) *_{\eta} P_{\Gamma}(.)
\end{aligned}
$$

Introducing the generating function $\hat{N}_{\Gamma}(z, \eta)=\sum_{k=0}^{+\infty} N_{\Gamma}(k, \eta) z^{k}$, we obtain using the fixed point solution $P_{\Gamma}(\eta)=e^{-\eta}$

$$
\left(\Gamma \partial_{\Gamma}-(1+\eta) \partial_{\eta}-1\right) \hat{N}_{\Gamma}(z, \eta)=2 \hat{N}_{\Gamma}(z, .) *_{\eta} e^{-\eta}-2 \hat{N}_{\Gamma}(z, \eta)+z \hat{N}_{\Gamma}(z, 0) \eta e^{-\eta}
$$

We look for a solution of the form

$$
\hat{N}_{\Gamma}(z, \eta)=\Gamma^{-\Phi(z)}(a(z)+b(z) \eta) e^{-\eta}
$$

and find a quadratic equation $(\Phi(z)-1)(\Phi(z)-2)=1+z$ so that

$$
\Phi(z)=\frac{3-\sqrt{5+4 z}}{2}
$$

Second method: this consists of associating with each bond a set of auxiliary variables $m(k)$ counting, respectively, the number of sites on the bond which have changed orientation exactly $k$ times since $t=0$. The RG rules for these variables upon decimation of bond (2) read (see Fig 2)

$$
\begin{aligned}
& m^{\prime}(k)=m_{1}(k)+m_{2}(k-1)+m_{3}(k) \\
& m^{\prime}(0)=m_{1}(0)+m_{3}(0) .
\end{aligned}
$$

Introducing the generating function $m(z)=\sum_{k=0}^{\infty} m(k) z^{k}$ one finds, for a fixed $z$ the $\mathrm{RG}$ rule

$$
m^{\prime}(z)=m_{1}(z)+z m_{2}(z)+m_{3}(z)
$$

A method to analyze such rules is to write the RG equation for the bond joint distribution $P(\eta, m)$. This is hard to solve, however the RG equation for the first moment $c(\eta)=\int_{m} m P(\eta, m)$ can be solved. Interestingly, the type of combination rule (64) under RSRG has been studied in [21] in the context of quantum spin chain models. We have recalled that analysis in the Appendix A, and generalized it to the recursion relation $m^{\prime}=a m_{1}+b m_{2}+c m_{3}$, which we will extensively need in the present problem. In particular, for $z=0$, which corresponds to computing the probability of no return to the origin $M_{0}(t)$, the rule is simply $m^{\prime}=m_{1}+m_{3}$. Remarkably, this is exactly the the same as the one for the magnetization in the RTFIC [21]. Thus there is an interesting relation between the magnetization of the RTFIC and persistence properties in the Sinai model. 
Since we are interested in the fraction of initial conditions with $k$ crossings, we need the ratio $\frac{m(z)}{\bar{l}_{\Gamma}}$ where the characteristic length $\bar{l}_{\Gamma}$ grows as $\Gamma^{2}$. The results from [21] and Appendix $\mathbb{A}$ is that the ratio $\frac{m(z)}{\bar{l}_{\Gamma}}$ decays as $\Gamma^{-\Phi(z)}$ with $\Phi(z)=$ $(3-\sqrt{5+4 z}) / 2$ in agreement with the first method (61).

Results: returns to the origin and multifractality: From the above result we can extract several consequences. First, setting $z=0$ we directly find that the probability that a thermally averaged trajectory does not return to its starting point decays, in terms of $\bar{l}(t) \sim(T \ln t)^{2}$, as:

$$
M_{0}(t) \sim \bar{l}(t)^{-\bar{\theta}} \quad \text { with } \quad \bar{\theta}=\frac{3-\sqrt{5}}{4} .
$$

Second, it is natural to introduce the rescaled number of returns to the origin:

$$
g=\frac{k}{\ln \Gamma}=\frac{k}{\ln (T \ln t)}
$$

and to define the generalized persistence exponent $\bar{\theta}(g)$ characterizing the asymptotic decay of the probability distribution of $g$ :

$$
\operatorname{Prob}(g) \sim \bar{l}(t)^{-\bar{\theta}(g)}
$$

We now compute $\bar{\theta}(g)$ from the above generating functional (60). By definition:

$$
\int_{0}^{\infty} d \eta \hat{N}_{\Gamma}(z, \eta) \propto \ln \Gamma \int_{0}^{\infty} d g z^{g \ln \Gamma} \Gamma^{-2 \bar{\theta}(g)}=\ln \Gamma \int_{0}^{\infty} d g e^{-\ln \Gamma(2 \bar{\theta}(g)-g \ln z)}
$$

Since we know that $N_{\Gamma}(z) \sim \Gamma^{-\Phi(z)}$ we then obtain, using the saddle point method, that $\Phi(z)=2 \bar{\theta}\left(g^{*}(z)\right)-g^{*}(z) \ln z$ where $g^{*}(z)$ is the solution of $2 \bar{\theta}^{\prime}\left(g^{*}(z)\right)=\ln z$. Properties of Legendre transforms thus give that, reciprocally, the exponent $\bar{\theta}(g)$ is given by $2 \bar{\theta}(g)=\Phi\left(z^{*}(g)\right)+g \ln z^{*}(g)$ where $z^{*}(g)$ is the solution of $\Phi^{\prime}\left(z^{*}(g)\right)=$ $-\frac{g}{z^{*}(g)}$. We find $z^{*}(g)=2 g\left(g+\sqrt{g^{2}+5 / 4}\right)$ and thus:

$$
\bar{\theta}(g)=\frac{g}{2} \ln \left[2 g\left(g+\sqrt{g^{2}+\frac{5}{4}}\right)\right]+\frac{3}{4}-\frac{g}{2}-\frac{1}{2} \sqrt{g^{2}+\frac{5}{4}} .
$$

The exponent $\bar{\theta}(g)$ is a positive convex function : it decays from $\bar{\theta}(g=0)=\frac{3-\sqrt{5}}{4}$ (for $g=0$ we of course recover the value found previously when studying the probability of no return to the origin up to $\Gamma$ ) to $\bar{\theta}\left(\frac{1}{3}\right)=0$, and then grows again for $g>1 / 3$. This implies that:

$$
g=\frac{k}{\ln (T \ln t)} \rightarrow \frac{1}{3} \quad \text { with probability } 1 \text { at large time }
$$

All of the moments of $\mathrm{g}$ will be dominated by the typical behavior; i.e. $\left\langle g^{m}\right\rangle \equiv$ $3^{-m}$ for all $\mathrm{m}$. The full dependence on $g$ of the $\bar{\theta}(g)$ function describes the tails of the probability distribution of the number of returns of $\langle x(t)\rangle$, i.e., the large deviations.

Returns to the origin for the running average: 
For a given walker, $\Xi(t) \equiv \frac{1}{t} \int_{0}^{t} x(\tau) d \tau$ will typically behave like $\langle x(t)\rangle$. We conjecture that the probability of $k=g \ln (\ln t)$ sign changes of $\Xi(t)$ up to time $t$ decays with the same exponent $\bar{\theta}(g)$ for $g \leq \frac{1}{3}$. For larger $g$, the behavior is dominated by rare valleys with closely spaced and almost degenerate minima on opposite sides of the origin which yield extra sign changes in $\Xi(t)$. We now estimate the contribution of these rare events.

We are interested in situations in which the number of zero crossings $N(t)$ of $y(t)=\int_{0}^{t} x(\tau) d \tau$ is much greater than those of $\langle x(t)\rangle$ whose statistics we know. The dominant contributions are from configurations of the random potential for which the valley bottom in which the origin lies is split in two halves on opposite side of the origin for a very long time. In such configurations the valley has two minima at $x_{+}>0$ and $x_{-}<0$ with a small free energy difference $T \epsilon$, separated by a barrier of some height $\Gamma_{0}$. The key point is that if the mean rate of change $\langle d y / d t\rangle \approx$ $\langle x\rangle_{\text {valley }} \approx\left(x_{+}+x_{-} e^{-\epsilon}\right) /\left(1+e^{-\epsilon}\right)$ happens to be very close to zero, $y(t)$ will change sign an anomalously large number of times. To estimate the corresponding number of crossings, one can consider, crudely, that $y(t)$ performs a biased random walk, with steps of order $x_{ \pm} e^{\Gamma_{0} / T}$ and an average drift $e^{\Gamma_{0} / T}\langle x\rangle_{\text {valley }}$ (since the typical time between jumps is $e^{\Gamma_{0} / T}$ ). Using well-known results for biased random walks, we can estimate that if the particle is trapped in the double valley for a time $\tau \gg e^{\Gamma_{0} / T}$, the typical number of zero crossings of $y(t)$ in that time interval will be:

$$
N(\tau) \sim \min \left(\frac{x_{ \pm}}{\langle x\rangle_{\text {valley }}}, \sqrt{\tau e^{-\Gamma_{0} / T}}\right)
$$

i.e., with $\tau$ cutting off a quantity inversely proportional to $\langle x\rangle_{\text {valley. But the dis- }}$ tribution of $w=\langle x\rangle_{\text {valley }} / x_{ \pm}$is constant near zero, with density of order $1 / \Gamma_{0}$, because of the distribution of $\epsilon$. Thus we can focus on valleys with the smallest $\Gamma_{0}$ since these will produce the largest number of crossings.

We must now estimate also the probability that such an atypical valley survives for a long time $\tau$. For that, we need that neither segment on either side of the origin be decimated in the RG for time $\tau$, which happens with probability $1 / \ln ^{2} \tau$. For the contribution of these to the distribution of $N(t)$ we thus have, ignoring constants:

$$
\begin{aligned}
\operatorname{Prob}(N(t)=N) & \sim \int^{t} \frac{d \tau}{\tau(\ln \tau)^{3}}\left[\int_{\tau^{-1 / 2}} d w \delta\left(N-\frac{1}{w}\right)+\int_{0}^{\tau^{-1 / 2}} d w \delta(N-\sqrt{\tau})\right] \\
& \sim \frac{1}{N^{2}(\ln N)^{2}} \theta(\sqrt{t}-N)
\end{aligned}
$$

the dominant contribution coming from the first term in the square bracket. The first moment of $N$ is (barely) finite, but higher moments grow with time as:

$$
\overline{N(t)^{\alpha}} \sim \frac{t^{\frac{\alpha-1}{2}}}{(\ln t)^{2}} .
$$

Thus these type of events completely dominate the distribution of the more-thantypical $N(t)$ tail. For $g \equiv N / \ln \ln t>1 / 3$ the distribution is therefore not multifractal. 
On the anomalously small $N$ side of the distribution, the type of events which might be troublesome appear to be rare enough not to cause Prob(g) for $g<1 / 3$ to differ from that using $\langle x(t)\rangle$ instead of $\Xi(t)$. The result (69) for $\langle x(t)\rangle$ should thus hold also for $\Xi(t)$ for $g<1 / 3$.

\section{B. Distribution of the sequence of returns to the origin: symmetric case}

We now study a more refined quantity concerning the statistics of returns to the origin of the thermal average $\langle x(t)\rangle$ in the Sinai model. It turns out to be possible to obtain the full probability distribution of the complete sequence of the times $\Gamma_{1}=T \ln t_{1}, \ldots \Gamma_{k}=T \ln t_{k}$ of successive returns to the origin. This is possible because of the remarkable property that every time the thermal packet crosses the origin, it "loses its memory" of the past.

We consider the probability $D_{\Gamma, \Gamma^{\prime}}(\eta)$ that a bond has barrier $\eta$ at $\Gamma$ and has had its last change of orientation at scale $\Gamma^{\prime}$. Its evolution equation reads

$$
\left(\Gamma \partial_{\Gamma}-(1+\eta) \partial_{\eta}-1\right) D_{\Gamma, \Gamma^{\prime}}(\eta)=2 P_{\Gamma}(0) D_{\Gamma, \Gamma^{\prime}}(.) *_{\eta} P_{\Gamma}(.)-2 P_{\Gamma}(0) D_{\Gamma, \Gamma^{\prime}}(\eta)
$$

with the initial condition at $\Gamma=\Gamma^{\prime}$ given by $D_{\Gamma^{\prime}, \Gamma^{\prime}}(\eta)=P_{\Gamma^{\prime}}(.) *_{\eta} P_{\Gamma^{\prime}}($.$) . As stated$ above, this initial condition is independent of previous history because at each decimation the bond is chosen afresh. Since $P_{\Gamma}(\eta)=e^{-\eta}$, it is natural to look for a solution of the form $D_{\Gamma, \Gamma^{\prime}}(\eta)=\left(A_{\Gamma, \Gamma^{\prime}}+B_{\Gamma, \Gamma^{\prime}} \eta\right) e^{-\eta}$. This is found in terms of $\alpha=\Gamma / \Gamma^{\prime}$ as:

$$
\begin{aligned}
& A_{\Gamma, \Gamma^{\prime}}=\frac{1}{\lambda_{+}-\lambda_{-}}\left(\alpha^{-\lambda_{-}}-\alpha^{-\lambda_{+}}\right) \quad \text { with } \quad \lambda_{ \pm}=\frac{3 \pm \sqrt{5}}{2} \\
& B_{\Gamma, \Gamma^{\prime}}=\frac{1}{\lambda_{+}-\lambda_{-}}\left[\left(\lambda_{+}-1\right) \alpha^{-\lambda_{-}}+\left(1-\lambda_{-}\right) \alpha^{-\lambda_{+}}\right]
\end{aligned}
$$

The probability for a bond to be decimated at $\Gamma$ given that its last decimation occurred at $\Gamma^{\prime}$ is $\rho\left(\Gamma, \Gamma^{\prime}\right)=-\partial_{\Gamma} \int_{0}^{\infty} d \eta D_{\Gamma, \Gamma^{\prime}}(\eta)=\frac{1}{\Gamma} \rho\left(\alpha=\Gamma / \Gamma^{\prime}\right)$ with:

$$
\rho(\alpha)=\frac{1}{\alpha} \frac{1}{\lambda_{+}-\lambda_{-}}\left(\alpha^{-\lambda_{-}}-\alpha^{-\lambda_{+}}\right)
$$

Thus we have obtained the probability distribution $\Pi_{t^{\prime}}(t)$ for the time $t$ of next return to the origin (in the effective dynamics) given that the last return was at a time $t^{\prime}$; this exhibits "aging behavior" in $\alpha=\frac{\ln t}{\ln t^{\prime}}$

$$
\Pi_{t^{\prime}}(t) d t=\frac{d t}{t \ln t} \frac{1}{\lambda_{+}-\lambda_{-}}\left(\left(\frac{\ln t}{\ln t^{\prime}}\right)^{-\lambda_{-}}-\left(\frac{\ln t}{\ln t^{\prime}}\right)^{-\lambda_{+}}\right) .
$$

For the sequence of successive returns, the picture we obtain is therefore very simple: the sequence of scales $\left\{\Gamma_{k}\right\}$ at which the successive changes of orientation of a given bond occur is a multiplicative Markovian process constructed with the simple rule $\Gamma_{k+1}=\alpha_{k} \Gamma_{k}$ where $\left\{\alpha_{k}\right\}$ are independent identically distributed random variables of probability distribution $\rho(\alpha)$. As a consequence, $\Gamma_{k}=\alpha_{k-1} \alpha_{k-2} \cdots \alpha_{2} \Gamma_{1}$ 
is simply the product of random variables, so that we obtain using the central limit theorem that

$$
\lim _{k \rightarrow \infty}\left(\frac{\ln \Gamma_{k}}{k}\right)=\langle\ln \alpha\rangle=3
$$

and we thus recover that the number $k$ of changes of orientation grows as $\ln \Gamma=$ $\ln \ln t$ and that the rescaled variable $g=\frac{k}{\ln (T \ln t)}$ is equal to $1 / 3$ with probability 1 , as in (70).

\section{Number of jumps up to time $t$ for the effective dynamics}

In this section we study the behavior of the total number of jumps of the thermal averaged position $\langle x(t)\rangle$ at large times. We introduce the number $m(n)$ of starting points on a bond such that the effective-i.e., $\langle x(t)\rangle$-walker jumps exactly $n$ times between 0 and $t$ for the effective dynamics. We will use $m$ to denote various auxiliary variables and trust that such local varying usage will not be confusing. The RG rules for these auxiliary variables upon decimation of bond 2 read (see Fig. 2)

$$
\begin{aligned}
& m^{\prime}(n)=m_{1}(n-1)+m_{2}(n-1)+m_{3}(n) \\
& m^{\prime}(0)=m_{3}(0)
\end{aligned}
$$

Introducing the generating function $m(z)=\sum_{n=0}^{\infty} m(n) z^{n}$ one finds the RG rule $m^{\prime}(z)=z m_{1}(z)+z m_{2}(z)+m_{3}(z)$. We thus find (see Appendix A) that the ratio $m(z) / \bar{l}_{\Gamma}$ decays as $\Gamma^{-\Phi(z)}$ where $\Phi(z)$ is now the solution of the equation:

$$
0=\left(\frac{\Phi(z)}{2}\right) U(-z, \Phi(z), 1)-U(-z, 1+\Phi(z), 1)
$$

in terms of the hypergeometric function $U(a, b, z)$.

Performing the same saddle point analysis as in the previous section we find that the rescaled variable $G=n / \ln \Gamma=n / \ln (T \ln t)$ for the number $n$ of jumps up to time $t$, has a multifractal distribution

$$
\operatorname{Prob}(G) \sim \bar{l}(t)^{-\omega(G)}
$$

where the exponent $\omega(G)$ is given by Legendre transform as $2 \omega(G)=\Phi\left(z^{*}(G)\right)+$ $G \ln z^{*}(G)$ where $z^{*}(G)$ is the solution of $\Phi^{\prime}\left(z^{*}(G)\right)=-G / z^{*}(G)$. Note that for $G=0$ (which corresponds to $z=0$ ) one has $\omega(G=0)=\Phi(z=0)=2$. As in the previous section, the asymptotic value $G_{a}$ that $G$ takes with probability one at large times is determined by the minimum of $\omega(G)$ where $\omega^{\prime}\left(G_{a}\right)=0$ which corresponds to $z^{*}\left(G_{a}\right)=1$. Thus $G_{a}=-z^{*}\left(G_{a}\right) \Phi^{\prime}\left(z^{*}\left(G_{a}\right)\right)=-\Phi^{\prime}(z=1)$. Differentiating (86) and using $\Phi(z=1)=0$ we find:

$$
G_{a}=\frac{U_{1}(-1,1,1)}{U(-1,0,1) / 2-U_{2}(-1,1,1)}=4 / 3
$$

where $U_{1}(a, b, z) \equiv \partial_{a} U(a, b, z)$ and $U_{2}(a, b, z) \equiv \partial_{b} U(a, b, z)$. 
A similar method can be used to compute the the joint distribution $P(G, g) \sim$ $\bar{l}(t)^{-\bar{\theta}(G, g)}$ of the two rescaled variables $G=n / \ln (T \ln t)$ and $g=k / \ln (T \ln t)$ where $n$ and $k$ are respectively the total number of jumps and the number of returns to the origin, and hence the associated decay exponent $\bar{\theta}(G, g)$. As an example of this application, we give the large time limit (valid with probability 1 ) of the the total rescaled number of jumps $G_{g}$, conditioned on a fixed rescaled number $g$ of returns to the origin:

$G_{g}=\frac{1+z(g)}{3-2 \Phi(z(g))}\left[U_{1}(-1,1+\Phi(z(g)), 1)+\frac{1-z(g)-\Phi(z(g))}{1+z(g)} U_{1}(-1, \Phi(z(g)), 1)\right]$

where $z(g)=2 g\left(g+\sqrt{g^{2}+\frac{5}{4}}\right)$ and $\Phi(z)=\frac{3-\sqrt{5+4 z}}{2}$. Note that for $g=1 / 3$ one recovers $G_{1 / 3}=4 / 3$ as expected.

\section{Correlations of the jumps}

In this section we will obtain some information about the statistical properties of the sequence of the directions and times of successive jumps. We will define a jump forward as a jump in the same direction as the previous one, and a jump backward as a jump in the opposite direction than the previous one. Note that a jump backward necessarily involves a return to the origin due to the properties of the $\mathrm{RG}$ procedure. The directions of successive jumps exhibit strong correlations since we have found in the previous sections that the total number of jumps behaves as $n \sim \frac{4}{3} \ln \ln t$, whereas the number of backward jumps (returns to the origin) behaves as $k \sim \frac{1}{3} \ln \ln t$. Thus in the effective dynamics the walker is substantially more likely to jump in the same direction as the previous jump. This is simply because the barrier of a bond which has just been created by decimation and the resulting combination of three bonds is higher than that of a typical bond at that scale and thus it is less likely to be decimated than the other bond encompassing the valley in which the walker rests.

We first compute the stationary distribution of the number of jumps forward made since the last return to the origin, i.e., the probabilities $\left\{c_{p}\right\}$ that a walker (at a given time) has made $p$ successive jumps forward since its last jump backward. This can be obtained by introducing $m(p)$ as the number of initial points on a bond such that the walker has jumped exactly $p$ times since the last passage over the origin for the effective dynamics, normalized as $\sum_{p=0}^{\infty} m(p)=l$ where $l$ is the length of the bond. The $\mathrm{RG}$ rules upon decimation of bond 2 read (see Fig. 2) $m^{\prime}(p)=m_{1}(p-1)+m_{3}(p), m^{\prime}(0)=l_{2}+m_{3}(0)$. The generating function thus has the rules $m^{\prime}(z)=z m_{1}(z)+l_{2}+m_{3}(z)$ where $l_{2}=\sum_{p=0}^{\infty} m_{2}(p)=m_{2}(z=1)$ is the length of bond (2). Similar methods as above then yield the generating function $c(z)=\sum_{p=0}^{\infty} c_{p} z^{p}$ :

$$
c(z)=\frac{U(-z,-1,1)+U(1-z, 0,1)}{3(U(-z, 0,1)+U(-z, 1,1))}
$$

in terms of hypergeometric functions, normalized to $c(z=1)=1$. From (86) one gets the $c_{p}$, e.g. $c_{0}=(1+U(1,0,1)) / 6=0.23394 \ldots, c_{1}=0.17492 \ldots, c_{2}=0.13356 \ldots$, 
$c_{3}=0.1029 \ldots, c_{4}=0.07958 \ldots$ etc. Since $c(z)$ has a pole for $z \approx 1.2884$, we obtain that

$$
c_{p} \sim \exp (-0.25343 p)
$$

for large $p$. Also, at any given (large) time the average number of jumps forward made since the last jump backward is $N_{\text {forward }}^{\text {av }}=c^{\prime}(z=1)=3.3975649 \ldots$

Next, we study the jump time dependence of backward and forward jumps. In Appendix C1] we compute the conditional probabilities $\rho_{\Gamma, \Gamma^{\prime}}^{f}\left(\right.$ respectively $\left.\rho_{\Gamma, \Gamma^{\prime}}^{b}\right)$ to make a forward jump (respectively backward) at $\Gamma$ given that the last jump occurred at $\Gamma^{\prime}$. These are scaling functions of the ratio $\alpha=\frac{\Gamma}{\Gamma^{\prime}}=\frac{\ln t}{\ln t^{\prime}}$, i.e., $\rho_{\Gamma, \Gamma^{\prime}}^{f, b} d \Gamma=\rho^{f, b}(\alpha) d \alpha$ with:

$$
\begin{aligned}
& \rho^{f}(\alpha)=\frac{1}{2 \alpha^{3}}\left(5-(\alpha+2) e^{-(\alpha-1)}\right) \\
& \rho^{b}(\alpha)=\frac{1}{2 \alpha^{3}}\left(5-\left(\alpha^{2}+2 \alpha+2\right) e^{-(\alpha-1)}\right) .
\end{aligned}
$$

Integrating over $\alpha$ we recover, as expected from (170) and (84), the total probabilities of the next jump begin a forward or a backward jump as $\rho^{f}=\int_{1}^{\infty} d \alpha \rho^{f}(\alpha)=\frac{3}{4}$ and $\rho^{b}=\frac{1}{4}$. Note that $\rho(\alpha)=\rho^{f}(\alpha)+\rho^{b}(\alpha)$ gives the total probability that the next jump $(f$ or $b)$ occurs at $\Gamma=\alpha \Gamma^{\prime}$.

We now study the statistical properties of the full sequence of the times of successive jumps $\left(\Gamma_{1}=T \ln t_{1}, \ldots \Gamma_{k}=T \ln t_{k}\right)$. Contrary to the sequence of the times of backward jumps studied in Section IVB which was simply a multiplicative Markovian process, there are persistent correlations in the full sequence of jumps which makes it much harder to analyze. Indexing each sequence by whether each jump is forward $(f)$ or backward $(b)$ we need to introduce the following set of conditional probabilities:

$$
\rho_{k}^{b f \ldots f b}\left(\Gamma_{k+1}, \Gamma_{k}, \ldots \Gamma_{1} \mid \Gamma_{0}\right)
$$

that, given that a backward jump occurred at $\Gamma_{0}$, there are exactly $k$ forward jumps occurring at times $\Gamma_{k}, \ldots \Gamma_{1}$ before the next backward jump occurs at $\Gamma_{k+1}$. These conditional probabilities are the elementary building blocks of the full measure for the sequence of jumps, since once a backward jump occurs, as was noted in Section IVB, the process starts afresh. Thus the full measure is simply a product of the above terms (89). We have computed the first terms of the set of conditional probabilities in the Appendix C2. We obtain, for instance, that, given that the previous jump was backward, the probability that the next jump is backward is $\frac{-e}{2} E i(-1)=0.298174$ and forward is 0.701826 . This result is different from above where we did not assume that the previous jump was backward.

\section{E. Number of returns to the origin: biased case}

We now study the returns to the origin of the thermal averaged position $\langle x(t)\rangle$ in the case where a small bias is applied. Then one expects that the number of returns is finite, since eventually the packet will leave the vicinity of the origin. However if 
the bias is small the number of return is large and universal results can be obtained in the limit $\delta$ small, $t$ large with $\gamma=\delta T \ln t$ fixed.

The method consists again in introducing auxiliary variables $m^{ \pm}(k)$ to count the number of initial points on a $( \pm)$ renormalized bond at $\Gamma=T \ln t$ which have changed exactly $k$ times orientation up to time $t$. The RG rules for these variables upon decimation of bond (2) are

$$
m^{ \pm}(k)=m_{1}^{ \pm}(k-1)+m_{2}^{\mp}(k-1)+m_{3}^{ \pm}(k)
$$

for $k \geq 1$ and $m^{ \pm}(0)=m_{3}^{ \pm}(0)$ for $k=0$. Introducing the generating functions $m^{ \pm}(z)=\sum_{k=0}^{\infty} m^{ \pm}(k) z^{k}$ one finds the RG rule

$$
m^{ \pm}(z)=m_{1}^{ \pm}(z)+z m_{2}^{\mp}(z)+m_{3}^{ \pm}(z) .
$$

The calculation of the mean value $\left\langle m^{ \pm}(z)\right\rangle$ is performed in Appendix B and gives:

$$
\left\langle m^{ \pm}(z)\right\rangle=\delta^{-\psi(z)}\left(A_{z}(\gamma)+e^{ \pm \gamma} \sinh (\gamma) \partial_{\gamma} A_{z}(\gamma)\right)
$$

with $\gamma=\delta \Gamma, \psi(z)=\frac{1}{2}(1+\sqrt{5+4 z})$ and $A_{z}(\gamma)=K_{z} Q_{\psi(z)-1}(\operatorname{coth} \gamma)$ in term of the Legendre function $Q_{\nu}(z)$. The constant $K_{z}$ depends a priori on $z$ in a nonuniversal way. From this we obtain the generating function of the probabilities $p_{\gamma}(k)=M_{k}(t)$ that the averaged position $\langle x(t)\rangle$ has returned exactly $k$ times to the origin up to time $t$. It is simply given (since initial conditions are uniformly distributed) as the generating function of the total number of initial conditions with $k$ returns divided by the total length and thus reads

$$
\sum_{k=0}^{\infty} p_{\gamma}(k) z^{k}=\frac{\left\langle m^{+}(z)\right\rangle+\left\langle m^{-}(z)\right\rangle}{\bar{l}_{\Gamma}}=\delta^{2-\psi(z)} \mathcal{M}_{z}(\Gamma \delta)
$$

with the scaling function:

$$
\mathcal{M}_{z}(\gamma)=\frac{2 K_{z}}{\sinh (\gamma)^{2}}\left[Q_{\psi(z)-1}(\operatorname{coth}(\gamma))-\operatorname{coth}(\gamma) Q_{\psi(z)-1}^{\prime}(\operatorname{coth}(\gamma))\right]
$$

and normalization implies that $K_{z=1}=1 / 2$. This function has the following asymptotic behaviors. For small $\gamma$ it behaves as a power law $\mathcal{M}_{z}(\gamma) \simeq$ $K_{z} \sqrt{\pi} 2^{1-\psi(z)} \frac{\Gamma[\psi(z)]}{\Gamma[\psi(z)+1 / 2]}(1+\psi(z)) \gamma^{\psi(z)-2}$ which allows one to recover the results of Section $\mathrm{IVA}$ in the limit of a vanishing bias $\delta \rightarrow 0$. For large $\gamma \rightarrow \infty$ it goes to a constant $\mathcal{M}_{z}(\infty)=K_{z}$ consistent with a finite total number of returns.

Setting $z=0$, we obtain the probability that $\langle x(t)\rangle$ has not returned to the origin up to time $t$ :

$$
M_{0}(t)=p_{\gamma}(k=0) \sim \delta^{2 \bar{\theta}} \mathcal{M}_{0}(\gamma)
$$

where the exponent $2 \bar{\theta}=\frac{3-\sqrt{5}}{2}$ coincides with the exponent $\beta$ of the magnetization of the RTFIC [21]. Note however, that although the scaling function $\mathcal{M}_{0}(\gamma)$ in the particular case of $z=0$, which corresponds to the probability of no return, is closely related to the scaling function of the magnetization of the RTFIC, it is not identical to it. The probability of no returns of the running average of $x, \Xi(t)$, will have the same asymptotic behavior as $\langle x(t)\rangle$. 
It is interesting to estimate the distribution of the total number of returns. This is achieved by studying the limit $\Gamma \rightarrow \infty$ in (93). We obtain that the probabilities $M_{k}(t)=p_{\gamma=\infty}(k)$, that the thermally averaged position $\langle x(t)\rangle$ return exactly $k$ times to the origin between $t=0$ and $t=+\infty$ have generating function

$$
\sum_{k=0}^{\infty} p_{\gamma=\infty}(k) z^{k}=\delta^{2-\psi(z)} \mathcal{M}_{z}(\infty)
$$

It is thus natural to introduce the rescaled variable $g=\frac{k}{(-\ln \delta)}$ and to look for the exponent characterizing the behavior for small $\delta$. One finds, by an analysis similar to that of Section IVA

$$
\operatorname{Prob}(g) \sim \delta^{2 \bar{\theta}(g)}
$$

with the same exponent $\bar{\theta}(g)$ and multifractal behavior as in (69). The same reasoning as in Section IVA leads to the result that $g$ is equal to $\frac{1}{3}$ with probability one for small $\delta$ and thus that the total number $k$ of returns to the origin in the presence of a small bias is

$$
k \approx \frac{|\ln \delta|}{3}
$$

\section{F. Distribution of the sequence of returns to the origin: biased case}

As in the symmetric case, it is possible to obtain the full probability distribution of the sequence $\left\{\Gamma_{1}, \Gamma_{2}, \ldots\right\}$ of successive returns to the origin. However in the case with drift in direction $(+)$, this sequence is finite with probability 1 since there is a finite probability that the particle never returns to the origin if it is on the right of its starting point. Therefore the probability $\Pi_{k}\left(\Gamma_{1} \ldots \Gamma_{k}\right)$ that the particle returns exactly $(k)$ times to the origin from $t=0$ to $t=\infty$ and that these returns take place at scale $\left(\Gamma_{1}, \ldots, \Gamma_{k}\right)$ can be decomposed as the product

$$
\Pi_{k}\left(\Gamma_{1} \ldots \Gamma_{2 k}\right)=\rho^{+}\left(\infty, \Gamma_{k}\right) \rho^{-}\left(\Gamma_{k}, \Gamma_{k-1}\right) \rho^{+}\left(\Gamma_{k-1}, \Gamma_{k-2}\right) \ldots
$$

where $\rho^{ \pm}\left(\Gamma, \Gamma^{\prime}\right)$ are the conditional probabilities that the particle returns to the origin at $\Gamma$ given that the last return to the origin occurred at scale $\Gamma^{\prime}$ in the direction $( \pm)$, and where $\rho^{+}\left(\infty, \Gamma^{\prime}\right)$ represents the probability that the particle never returns to the origin after the last passage to the origin occurred at $\Gamma^{\prime}$ in the $(+)$ direction

$$
\rho^{+}\left(\infty, \Gamma^{\prime}\right)=1-\int_{\Gamma^{\prime}}^{\infty} d \Gamma \rho^{+}\left(\Gamma, \Gamma^{\prime}\right)
$$

We have computed these probabilities in Appendix D. They are most naturally written in terms of the reduced variables $y=\operatorname{coth} \gamma, y^{\prime}=\operatorname{coth} \gamma^{\prime}$ with $1<y<y^{\prime}$ (where $\gamma=\delta \Gamma=T \delta \ln t$ and $\gamma^{\prime}=\delta \Gamma^{\prime}=T \delta \ln t^{\prime}$ ) as:

$$
\rho^{ \pm}\left(\Gamma, \Gamma^{\prime}\right) d \Gamma=\tilde{\rho}^{ \pm}\left(y, y^{\prime}\right) d y=\frac{y \mp 1}{y^{\prime} \pm 1}\left[Q_{\phi-1}(y) P_{\phi-1}\left(y^{\prime}\right)-Q_{\phi-1}\left(y^{\prime}\right) P_{\phi-1}(y)\right] d y
$$


with $\phi=\frac{1}{2}(1+\sqrt{5})$ and $P_{\nu}$ and $Q_{\nu}$ Legendre functions. One finds, as expected, that $\int_{1}^{y^{\prime}} d y \tilde{\rho}^{-}\left(y, y^{\prime}\right)=1$. On the other hand, the probability that the thermally averaged position $\langle x(t)\rangle$ never crosses the origin again after having crossed it at $\Gamma^{\prime}$ is:

$$
\rho^{+}\left(\infty, \Gamma^{\prime}\right)=1-\int_{\Gamma^{\prime}}^{\infty} d \Gamma \rho^{+}\left(\Gamma, \Gamma^{\prime}\right)=2 \frac{P_{\phi-1}\left(y^{\prime}\right)}{y^{\prime}+1}
$$

For $\gamma^{\prime} \rightarrow 0$ this probability vanishes as:

$$
\rho^{+}\left(\infty, \Gamma^{\prime}\right)=2 \frac{\Gamma\left(\phi-\frac{1}{2}\right)}{\sqrt{\pi} \Gamma(\phi)} \gamma^{\prime 2-\phi}
$$

while it goes to 1 for large $\gamma^{\prime}$ as:

$$
\rho^{+}\left(\infty, \Gamma^{\prime}\right)=1-\frac{e^{-4 \gamma^{\prime}}}{4}=1-\frac{1}{4 t^{2 \mu}}
$$

where $\mu=2 \delta T$. The factor $1 / t^{2 \mu}$ can be understood with a simple argument. Since the particle having crossed the origin at $t^{\prime}$ belongs to a renormalized bond just being created, its barrier is distributed not with $P_{\Gamma^{\prime}}^{+}(\zeta)(22)$, but with $P_{\Gamma^{\prime}}^{\text {new }}(\zeta)=$ $P_{\Gamma^{\prime}}^{+}(.) *_{\zeta} P_{\Gamma^{\prime}}^{+}()=.\left(u_{\Gamma^{\prime}}^{+}\right)^{2} \zeta e^{-u_{\Gamma^{\prime}}^{+} \zeta} \sim \zeta / t^{\prime 2 \mu} e^{-\zeta \mu /\left(T t^{\prime \mu}\right)}$; this is depleted near the origin which is the key point. For a return to occur after $t^{\prime}$, the new renormalized bond has to be decimated in the future, and the dominant contribution comes from the times near $t^{\prime}$, i.e., we have to compute the probability that the two independent barriers of the neighboring bonds each distributed with $P_{\Gamma^{\prime}}^{-}$are bigger than the new $(+)$ renormalized bond at $\Gamma^{\prime}$ : this probability is simply $\frac{\left(u_{\Gamma^{\prime}}^{+}\right)^{2}}{\left(u_{\Gamma^{\prime}}^{+}+2 u_{\Gamma^{\prime}}^{-}\right)^{2}}$ and thus behaves as $1 /\left(4 t^{\prime 2 \mu}\right)$ at large $t^{\prime}$. These events are thus responsible for the dominant behavior of $\rho^{+}\left(\infty, \Gamma^{\prime}\right)$ found above.

\section{RETURN TO THE ORIGIN OF A SINGLE WALKER, FIRST PASSAGE TIMES AND MEETING TIME OF TWO WALKERS}

\section{A. Probability of no return to the origin for a single walker}

We now compute, in the presence of a small bias, the probability $N^{+}(t)$ (respectively $N^{-}(t)$ ) that a single walker has remained all the time to the right (respectively to the left) of its starting point - the bias being by convention to the right. These probabilities are found by placing an absorbing boundary at $x=0$ as discussed in Section IIB3. We note that the probability distributions $E_{\Gamma}^{ \pm}(l)$ of the length $l$ of the absorbing zone satisfying the RG equation (10) has initial condition $\delta(l-1)$ (counting the first infinitely deep bond in Fig. 3 as length 1 by convention) and it is the weight of this $\delta$-function part that determines the desired no-return probability. For finite $\Gamma, E_{\Gamma}^{ \pm}(l)$ takes the form:

$$
E_{\Gamma}^{ \pm}(l)=\delta(l-1) \int_{0}^{+\infty} d \zeta R_{\Gamma}^{ \pm}(\zeta)+\text { regular part }
$$

where $R_{\Gamma}^{ \pm}(\zeta)$ is the probability that the first descending bond (in Fig. 3 for the $(+)$ case) has never been decimated up to scale $\Gamma$ and has barrier $F=\Gamma+\zeta$ at $\Gamma$. The 
total weight of the delta function $r_{\Gamma}^{ \pm}=\int_{0}^{+\infty} d \zeta R_{\Gamma}^{ \pm}(\zeta)$ decays to zero as the regular part of $E_{\Gamma}^{ \pm}(l)$ converges towards the fixed point determined in Section IC3. We obtain the probabilities $N^{ \pm}(t)$ from $N^{ \pm}(t) \sim r_{\Gamma=T \ln t}^{ \pm}$.

The evolution equation for $R_{\Gamma}^{ \pm}(\zeta)$ reads

$$
\left(\partial_{\Gamma}-\partial_{\zeta}\right) R_{\Gamma}^{ \pm}(\zeta)=-P_{\Gamma}^{\mp}(0) R_{\Gamma}^{ \pm}(\zeta)+P_{\Gamma}^{\mp}(0) R_{\Gamma}^{ \pm}(.) *_{\zeta} P_{\Gamma}^{ \pm}(.)
$$

Setting $R_{\Gamma}^{ \pm}(\zeta)=r_{\Gamma}^{ \pm} P_{\Gamma}^{ \pm}(\zeta)$ we obtain $\partial_{\Gamma} \ln \left(r_{\Gamma}^{ \pm}\right)=-u_{\Gamma}^{ \pm}=\partial_{\Gamma} \ln \left(u_{\Gamma}^{\mp}\right)$ where we denote $u_{\Gamma}^{ \pm}=u_{\Gamma}^{ \pm}(0)$ in (19). Thus $r_{\Gamma}^{ \pm} \sim u_{\Gamma}^{\mp}$ which yields:

$$
\begin{aligned}
N^{+}(t) & \sim \frac{2 \delta}{1-e^{-2 \delta \Gamma}} \\
N^{-}(t) & \sim \frac{2 \delta}{e^{2 \delta \Gamma}-1}
\end{aligned}
$$

In the biased case, one finds that there is a finite probability $N^{+}(t) \sim 2 \delta$ of never returning to the origin if the particle starts in the direction of the drift, whereas if it starts against the drift, the probability of not returning to the origin up to time $t$ decays as $N^{-}(t) \sim 2 \delta e^{-2 \delta \Gamma} \propto t^{-\mu}$. This corresponds to the probability that the origin happens to belong to a "trap" impeding the drift of waiting time larger than $t$, as was discussed in [14]. We note that the calculation of these persistent probabilities in the Sinai model is similar to the calculation of the endpoint magnetization in the RTFIC [21].

In the symmetric case $\delta=0$ we thus find that the probability $N(t)$ that a single walker has never crossed its starting point $x(0)=x_{0}$ between 0 and $t$ decays at large time as

$$
N(t) \sim \bar{l}(t)^{-\theta} \quad \text { with } \theta=\frac{1}{2}
$$

with $\bar{l}(t)=(T \ln t)^{2}$. Note that the persistence exponent obtained here in the presence of disorder is different from the result $\theta_{\text {pure }}=1$ for the pure diffusion problem where the probability of no return to the origin up to time $t$ decays simply as $\frac{1}{\bar{l}(t)} \sim \frac{1}{\sqrt{t}}$. It is also significantly larger than the persistence exponent $\bar{\theta}=\frac{3-\sqrt{5}}{4}=$ 0.190983.. for thermally averaged trajectories obtained in Section IVA.

\section{B. First passage times in an infinite sample}

We now compute the distribution of the first passage time $T_{x_{0}}$ at $x=0$ of a walker which start at $x=x_{0}$ at $t=0$. The method consists in placing an absorbing boundary at $x=0$ and studying the probability $S_{x_{0}}(\Gamma)$ that this walker has survived up to scale $\Gamma=T \ln t$ as illustrated in (4). We use the method of decimation in the presence of a boundary discussed in Section $\amalg$ B 3. The probability $S_{x_{0}}^{ \pm}(\Gamma)$ that the walker starting at $x_{0}$ is still alive at $\Gamma$ in the presence of a $( \pm)$ drift is equal to the probability that the length $l_{1}$ of the absorbing zone in near the boundary (see Figure 3) is smaller than $x_{0}$ at $\Gamma$, which is:

$$
S_{x_{0}}^{ \pm}(\Gamma)=\int_{0}^{x_{0}} d l_{1} E_{\Gamma}^{\mp}\left(l_{1}\right)
$$


in terms of the function $E_{\Gamma}^{\mp}\left(l_{1}\right)$ studied in Section 【IC3. The probability that the first passage time $T_{x_{0}}$ is such that $\Gamma<T \ln T_{x_{0}}<\Gamma+d \Gamma$ is equal to the probability $\sigma_{x_{0}}^{ \pm}(\Gamma)$ that the walker is absorbed between $\Gamma$ and $\Gamma+d \Gamma$ and is simply obtained:

$$
\sigma_{x_{0}}^{ \pm}(\Gamma)=-\partial_{\Gamma} S_{x_{0}}^{ \pm}(\Gamma)=-\partial_{\Gamma} \int_{0}^{x_{0}} d l E_{\Gamma}^{\mp}(l)
$$

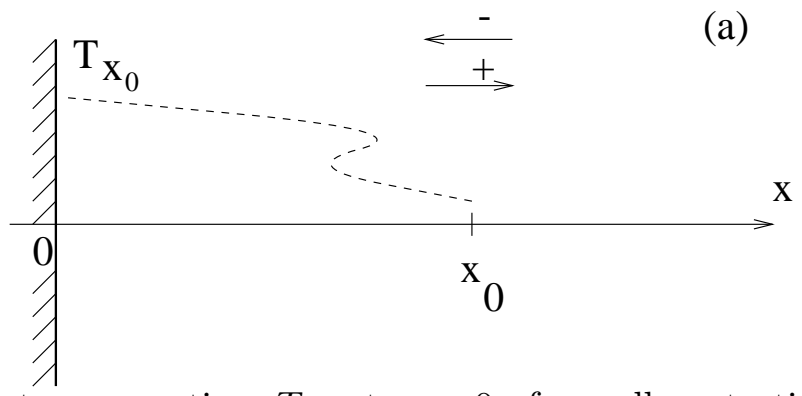

FIG. 4. The first passage time $T_{x_{0}}$ at $x=0$ of a walker starting at $x_{0}$ is obtained from the survival probability in the presence of an absorbing boundary at $x=0$.

\section{Symmetric case}

In the symmetric case $\delta=0$ we can rewrite (111) in terms of the distribution $E(\lambda)$ of the rescaled variable $\lambda=\frac{l_{1}}{\Gamma^{2}}$ given by (37) and obtain

$$
\sigma_{x_{0}}(\Gamma)=-\partial_{\Gamma} \int_{0}^{\frac{x_{0}}{\Gamma^{2}}} d \lambda E(\lambda)=\frac{2 x_{0}}{\Gamma^{3}} E\left(\frac{x_{0}}{\Gamma^{2}}\right) .
$$

The distribution of first passage time $T_{x_{0}}$ is naturally obtained in terms of the rescaled variable $w=T \ln T_{x_{0}} / \sqrt{x_{0}}$ which is a random variable distributed as

$$
s(w)=\frac{2}{w^{3}} E\left(\frac{1}{w^{2}}\right)=\frac{2}{w^{3}} \sum_{n=-\infty}^{+\infty} e^{-\frac{\pi^{2}}{w^{2}}\left(n+\frac{1}{2}\right)^{2}}=\frac{2}{\sqrt{\pi} w^{2}} \sum_{m=-\infty}^{+\infty}(-1)^{m} e^{-m^{2} w^{2}} .
$$

This distribution has the following asymptotic behavior. It behaves as $s(w) \sim$ $\left(4 / w^{3}\right) \exp \left[-\pi^{2} /\left(4 w^{2}\right)\right]$ for small $w$ as the smaller passage times are strongly suppressed. However it has a broad tail for large $w$ and decays as $s(w) \sim \frac{2}{\sqrt{\pi} w^{2}}$. In particular its first moment diverges: $\bar{w}=T \overline{\ln T_{x_{0}}} / \sqrt{x_{0}}=+\infty$. One can relate this tail to the result of the previous Section concerning the probability $N(t)$ that the walker never crosses 0 . In general one expects that $N(t) \sim C\left(x_{0}\right) / \Gamma$ for a walker starting at $x_{0}$, and that $C\left(x_{0}\right)$ is non-universal for fixed $x_{0}$. Here we find coming from the other limit in the scaling regime $x_{0} / \Gamma^{2}$ fixed but small, that the behavior of $C\left(x_{0}\right)$ at large $x_{0}$ should be universal as $C\left(x_{0}\right) \sim \sqrt{x_{0}}$ since we find here $N(t) \sim \int_{\Gamma / \sqrt{x_{0}}}^{+\infty} d w / w^{2} \sim \sqrt{x_{0}} / \Gamma$. 


\section{Biased case}

The Laplace transform with respect to $x_{0}$ of the survival probability reads

$$
\int_{0}^{\infty} d x_{0} e^{-p x_{0}} S_{x_{0}}^{ \pm}(\Gamma)=\frac{E_{\Gamma}^{\mp}(p)}{p}=\frac{u_{\Gamma}^{\mp}}{p u_{\Gamma}^{\mp}(p)} .
$$

Introducing again the scaling variables $\gamma=\delta \Gamma, X_{0}=\frac{x_{0}}{\Gamma^{2}}$, the Laplace inversion gives

$$
S_{x_{0}}^{ \pm}(\Gamma)=1-\sum_{n=0}^{\infty} C_{n}^{\mp}(\gamma) e^{-X_{0} s_{n}^{\mp}(\gamma)}
$$

where $s_{n}^{ \pm}(\gamma)$ have been introduced in (50), and where

$$
C_{n}^{ \pm}(\gamma)=\left(\frac{\gamma}{\sinh \gamma}\right) \frac{2\left(\alpha_{n}^{ \pm}(\gamma)\right)^{2} e^{\mp \gamma}}{\left(\gamma^{2}+\left(\alpha_{n}^{ \pm}(\gamma)\right)^{2}\right)\left(\gamma^{2}+\left(\alpha_{n}^{ \pm}(\gamma)\right)^{2} \mp \gamma\right)}
$$

except for the term $n=0$ in the domain $(+)$ and $\gamma>1$ where

$$
C_{0}^{+}(\gamma>1)=\left(\frac{\gamma}{\sinh \gamma}\right) \frac{2\left(\tilde{\alpha}_{0}^{+}(\gamma)\right)^{2} e^{-\gamma}}{\left(\gamma^{2}-\left(\tilde{\alpha}_{0}^{+}(\gamma)\right)^{2}\right)\left(\gamma+\left(\tilde{\alpha}_{0}^{+}(\gamma)\right)^{2}-\gamma^{2}\right)}
$$

For $\gamma \gg 1$, we find using (54) that the survival probability $S_{x_{0}}^{-}(\Gamma)$ in the presence of a bias towards the absorbing boundary has a simple exponential dependence on $x_{0}$

$$
S_{x_{0}}^{-}\left(\Gamma \gg \frac{1}{\delta}\right) \simeq 1-e^{-x_{0} 4 \delta^{2} e^{-2 \gamma}}=1-e^{-x_{0} \mu^{2} /\left(T^{2} t^{\mu}\right)}
$$

whereas in the case of a bias in the direction away from the absorbing boundary, we find

$$
S_{x_{0}}^{+}\left(\Gamma \gg \frac{1}{\delta}\right) \simeq 1-e^{-x_{0} \delta^{2}} \sum_{n=0}^{\infty} \frac{4 \gamma n^{2} \pi^{2}}{\left(\gamma^{2}+n^{2} \pi^{2}\right)\left(\gamma^{2}+n^{2} \pi^{2}+\gamma\right)} e^{-\frac{x_{0}}{\Gamma^{2}} n^{2} \pi^{2}} .
$$

In the limit $\Gamma \rightarrow \infty$, we obtain the probability that a particle reaches the point at a distance $x_{0}$ from its starting point in the far region against the drift

$$
\lim _{\Gamma \rightarrow \infty}\left(1-S_{x_{0}}^{+}(\Gamma)\right)=\frac{1}{\sqrt{\pi}\left(x_{0} \delta^{2}\right)^{3 / 2}} e^{-x_{0} \delta^{2}}
$$

which coincides, in the limit $\mu=2 \delta T \rightarrow 0$, with the exact result in the regime $0<\mu<2$ - corresponding to anomalous diffusion - [28, 44 which reads (for $T=1$ ): $\pi^{3 / 2} \frac{\Gamma\left(\frac{\mu}{2}\right)^{2}}{\Gamma(\mu)} \frac{1}{1-\cos (\pi \mu)} x_{0}^{-3 / 2} e^{-\mu^{2} x_{0} / 4}$. 


\section{Distribution of the maximum position}

The above calculation also yields the distribution of the maximum position $x_{\max }(t)=\max _{0<t^{\prime}<t} x\left(t^{\prime}\right)$ for a particle starting from $x=0$ at $t=0$ in the presence of $\mathrm{a}+$ (i.e., along the positive direction) or - bias:

$$
\operatorname{Prob}^{ \pm}\left(x_{\max }(t) \leq x_{m}\right)=S_{x_{m}}^{\mp}(\Gamma=T \ln t)=\int_{0}^{x_{m}} d l E_{\Gamma=T \ln t}^{ \pm}(l)
$$

and thus the boundary probabilities defined in (37, 35) $E_{\Gamma=T \ln t}^{ \pm}\left(x_{m}\right)$ correspond exactly to the distribution of the maximum of the Sinai walk.

In the symmetric case we thus recover via the $\mathrm{RG}$ a result derived by Golosov [27]. It is given in explicit form in (37). In addition we obtain, in the presence of a small bias, the explicit form:

$$
\operatorname{Prob}^{ \pm}\left(x_{\text {max }}(t)=x_{m}\right)=E_{\Gamma=T \ln t}^{ \pm}\left(x_{m}\right)=\sum_{n=0}^{+\infty} C_{n}^{ \pm}(\gamma) s_{n}^{ \pm}(\gamma) e^{-x_{m} s_{n}^{ \pm}(\gamma)}
$$

with the conventions of the previous section.

\section{Probability that two particules do not meet up to time $t$}

In this section we compute the distribution of the meeting time $T_{L}$ for two particules starting respectively, at $x=0$ and $x=L$. The RSRG method is well suited to compute this quantity which may be hard to get by other means. We call 1 and 2 the two particles, 1 starting from $x=0$ at $t=0$ and 2 from $x=L$. We compute the probability $F_{L}(\Gamma)$ that the two particles have not yet met at time $t$ with $\Gamma=T \ln t$. The probability $1-F_{L}(\Gamma)$ that they have met is equal to the probability that the segment $[0, L]$ is included in a single renormalized valley at $\Gamma$. The distribution $V_{\Gamma}(l)$ of the length $l$ of the valleys is given as $V_{\Gamma}(l)=P_{\Gamma}^{+} *_{l} P_{\Gamma}^{-}$. The probability that both 0 and $L$ belong to the same valley at scale $\Gamma$ is simply:

$$
1-F_{L}(\Gamma)=\frac{1}{\bar{l}_{\Gamma}} \int_{L}^{+\infty} d l(l-L) V_{\Gamma}(l) .
$$

This leads to the following expression for the Laplace transform with respect to $L$

$$
\int_{0}^{\infty} d L e^{-p L} F_{L}(\Gamma)=\frac{1-P_{\Gamma}^{+}(p) P_{\Gamma}^{-}(p)}{p^{2} \bar{l}_{\Gamma}}=\left(\frac{\delta^{2}}{p \sinh ^{2}(\Gamma \delta)}\right) \frac{\sinh ^{2}\left(\Gamma \sqrt{p+\delta^{2}}\right)}{p \cosh ^{2}\left(\Gamma \sqrt{p+\delta^{2}}\right)+\delta^{2}}
$$

In the symmetric case $\delta=0$, we find $F_{L}(\Gamma)=f\left(\lambda=\frac{L}{\Gamma^{2}}\right)$ with the scaling function

$$
\begin{aligned}
f(\lambda)=L T_{s \rightarrow \lambda}^{-1}\left(\frac{\tanh ^{2}(s)}{s^{2}}\right) & =\int_{0}^{\lambda} d x \sum_{-\infty}^{+\infty}\left(2 x+\frac{1}{\pi^{2}\left(n+\frac{1}{2}\right)^{2}}\right) e^{-x \pi^{2}\left(n+\frac{1}{2}\right)^{2}} \\
& =\sum_{-\infty}^{+\infty}\left[3 \frac{1-e^{-\lambda \pi^{2}\left(n+\frac{1}{2}\right)^{2}}}{\pi^{4}\left(n+\frac{1}{2}\right)^{4}}-2 \frac{\lambda e^{-\lambda \pi^{2}\left(n+\frac{1}{2}\right)^{2}}}{\pi^{2}\left(n+\frac{1}{2}\right)^{2}}\right] .
\end{aligned}
$$


The probability density $H_{L}(\Gamma)$ that the two particles meet between $\Gamma$ and $\Gamma+d \Gamma$ is $H_{L}(\Gamma)=-\partial_{\Gamma} F_{L}(\Gamma)$. We thus obtain that the meeting time is $T_{L}=\exp [w \sqrt{L} / T]$ where $w=\Gamma_{L} / \sqrt{L}$ is a random variable distributed as

$$
h(w)=\frac{2}{w^{3}} f^{\prime}\left(\frac{1}{w^{2}}\right)=\frac{2}{w^{3}} \sum_{-\infty}^{+\infty}\left(\frac{2}{w^{2}}+\frac{1}{\pi^{2}\left(n+\frac{1}{2}\right)^{2}}\right) e^{-\frac{\pi^{2}}{w^{2}}\left(n+\frac{1}{2}\right)^{2}}
$$

Note that in the effective dynamics, once the two particle meet they remain together at all later times. In the real dynamics, rare events as explored in Section VIH, can cause them to again split for a limited amount of time in distinct wells separated by a distance of order $\ln ^{2} t$, with a probability of order $1 / \ln t$.

\section{TWO TIME DIFFUSION FRONT IN THE SINAI MODEL AND AGING PROPERTIES}

In this section we will study the two-time quantity

$$
P\left(x, t, x^{\prime}, t^{\prime}\right) \equiv \overline{\operatorname{Prob}\left(x t, x^{\prime} t^{\prime} \mid 00\right)}
$$

i.e., the probability, over the ensemble of random landscapes and thermal fluctuations, that a particle starting at $x=0$ at $t=0$, be successively at $x^{\prime}$ at $t^{\prime}$ and at $x$ at $t$. Note that it is normalized as $\int d x^{\prime} d x P\left(x, t, x^{\prime}, t^{\prime}\right)=1$ and thus it is different - due to the averaging over the landscape - than the conditional probability $\overline{\operatorname{Prob}\left(x t \mid x^{\prime} t^{\prime}, 00\right)}$ that the particle be at $x$ at $t$, knowing that it was at $x^{\prime}$ at $t^{\prime}$ and at $x=0$ at $t=0$.

The average of the two-time probability contains a lot of information about the dynamics after letting the system evolve from $t=0$ to $t=t^{\prime} \equiv t_{w}$, i.e., the aging dynamics. We study $P\left(x, t, x^{\prime}, t^{\prime}\right)$ in the limit where both $t$ and $t^{\prime}$ are large. There are several time regimes, according to the precise way that the double limit $t^{\prime}, t \rightarrow \infty$ is taken, and we obtain analytic expressions for the scaling form of $P\left(x, t, x^{\prime}, t^{\prime}\right)$ in each of these regimes. We also study

$$
Q\left(y, t, t^{\prime}\right) \equiv \int d x^{\prime} P\left(x^{\prime}+y, t, x^{\prime}, t^{\prime}\right)
$$

i.e., the distribution of the displacements $y=x-x^{\prime}$ between $t^{\prime}$ and $t$. Finally, as explained below in VIA, we simultaneously obtain results for a "two-temperature" evolution.

Some properties of the quantity $P\left(x, t, x^{\prime}, t^{\prime}\right)$ were investigated previously in [16], by a numerical simulation and qualitative arguments. Here we obtain instead detailed exact results for this quantity. Whenever they can be compared these results are found in agreement with the conclusions of [16].

Before presenting the analytical results, let us first give a discussion of the various regimes studied in the following sections. 


\section{A. Discussion of the various regimes}

One can distinguish two main regimes for large $t$ and $t^{\prime}$, which we discuss in the symmetric case.

(i) "scaling regime": $t-t^{\prime} \sim t \sim t^{\prime \alpha}$, with $\alpha>1$ :

This first regime is $t \sim t^{\prime \alpha}$ with a fixed $\alpha=\frac{\ln t}{\ln t^{\prime}}>1$. This regime was called the "diffusion regime" in [16]. In this regime, typically the bond containing the origin can been decimated between $\Gamma^{\prime}=T \ln t^{\prime}$ and $\Gamma=T \ln t$ and thus motion can occur. $P\left(x, t, x^{\prime}, t^{\prime}\right)$, obtained below by iterating the $\mathrm{RG}$ from $\Gamma^{\prime}$ to $\Gamma$, takes a scaling form in the rescaled position variables. We thus define

$$
X=\frac{x}{\Gamma^{2}}
$$

and since there are two possible choices for the rescaled $x^{\prime}$, we define

$$
X^{\prime}=\frac{x^{\prime}}{\Gamma^{\prime 2}}
$$

and

$$
\tilde{X}^{\prime}=\frac{x^{\prime}}{\Gamma^{2}}=X^{\prime} \alpha^{2}
$$

Choosing to scale $x$ and $x^{\prime}$ by the same factor $\Gamma^{2}$, the scaling form $P_{\alpha}\left(X, \tilde{X}^{\prime}\right)$ for the two-time probability distribution reads:

$$
P\left(x, t, x^{\prime}, t^{\prime}\right) \sim \frac{1}{(T \ln t)^{4}} \quad P_{\alpha=\frac{\ln t}{\ln t^{\prime}}}\left(X=\frac{x}{(T \ln t)^{2}}, \tilde{X}^{\prime}=\frac{x^{\prime}}{(T \ln t)^{2}}\right) .
$$

This diffusion front simplifies in the two limits $\ln t \approx \ln t^{\prime}(\alpha=1)$ and $\ln t \gg \ln t^{\prime}$ $(\alpha \rightarrow+\infty)$. First for $\Gamma=\Gamma^{\prime}$ one must have:

$$
P_{\alpha=1}\left(X, \tilde{X}^{\prime}\right)=q\left(\tilde{X}^{\prime}\right) \delta\left(X-\tilde{X}^{\prime}\right)
$$

where $q\left(\tilde{X}^{\prime}\right)$ is the Kesten distribution (39) obtained previously (note that $X^{\prime}=\tilde{X}^{\prime}$ for $\alpha=1)$. An interesting feature is that the delta function component of the two-time diffusion front at $x=x^{\prime}$ persists even for $\ln t>\ln t^{\prime}$ :

$$
P_{\alpha}\left(X, \tilde{X}^{\prime}\right)=D_{\alpha}\left(\tilde{X}^{\prime}\right) \delta\left(X-\tilde{X}^{\prime}\right)+\tilde{P}_{\alpha}\left(X, \tilde{X}^{\prime}\right)
$$

where $\tilde{P}$ is a smooth function of its arguments. This property was suggested in [16]. Here we find that it arises naturally in the RSRG, since there is a finite probability that the bond which contains the origin (starting point) will have its lowest point unchanged by the renormalization between $\Gamma^{\prime}$ and $\Gamma$ (note that the bond can grow but only on one side).

This implies that a finite fraction of particles, $D\left(t, t^{\prime}\right)=D_{\alpha} \equiv \int_{\tilde{X}^{\prime}} D_{\alpha}\left(\tilde{X}^{\prime}\right)$ remain at the bottom of a valley (their renormalized valley at $\Gamma^{\prime}$ ) and do not move appreciably (i.e., by less than $O\left(\ln ^{2} t\right)$ ) between $t^{\prime}$ and $t$.

Finally, for very separated times, i.e., large $\alpha$, the time evolutions of $X$ at $t$ and $X^{\prime}$ at $t^{\prime}$ decouple and one recovers again the Kesten distribution (39). One has: 


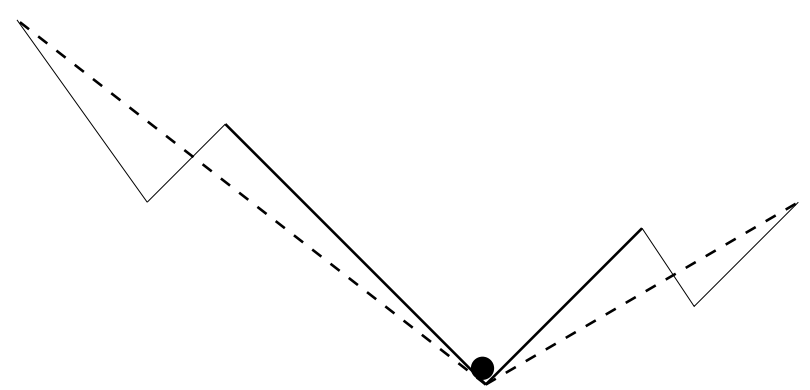

FIG. 5. Fraction of walkers which do not move between $t^{\prime}$ and $t$.

$$
D_{\alpha \rightarrow \infty}\left(\tilde{X}^{\prime}\right) \rightarrow 0 \quad \tilde{P}_{\alpha \rightarrow \infty}\left(X, \tilde{X}^{\prime}\right) \rightarrow \alpha^{2} q\left(\alpha^{2} \tilde{X}^{\prime}\right) q(X)
$$

In the next section $\mathrm{VIB}$ we will explicitly compute $D_{\alpha}\left(\tilde{X}^{\prime}\right)$. In section VID we will compute the full smooth part, which is more complicated.

(ii) "quasi-equilibrium regime": $t-t^{\prime} \sim t^{\prime \alpha}, \alpha<1$ :

The second regime is for $t-t^{\prime} \sim t^{\prime \alpha}$ with fixed $\alpha=\frac{\ln \left(t-t^{\prime}\right)}{\ln t^{\prime}}<1$. [This definition of $\alpha$ is consistent with the previous one in Ref. [16.]

In the second regime, the typical situation is that the thermal packet at $t^{\prime}$ is well equilibrated in a valley with the packet of width of $O(1)$. In this regime, there is typically no motion on scales larger than $O(1)$ between $t^{\prime}$ and $t$ as the particle is near the bottom of a valley. Motion on larger scales will thus be dominated by rare events, which we now analyze.

First, there is some probability that the valley to which the origin belongs undergoes a decimation resulting in a jump between $t^{\prime}$ and $t$. Although this jump is large (the walker will jump to the bottom of a deeper valley a distance of order $\bar{l}\left(\Gamma^{\prime}\right) \sim \Gamma^{\prime 2}$ away) the probability that it occurs is of order the probability that one of the barriers of the valley at time $t^{\prime}$ is less than $\Gamma$, which is itself of order $\left(\Gamma-\Gamma^{\prime}\right) / \Gamma^{\prime} \sim t^{\prime 1-\alpha} \sim \exp \left(-(1-\alpha) \Gamma^{\prime}\right)$ in this regime and thus negligible. The

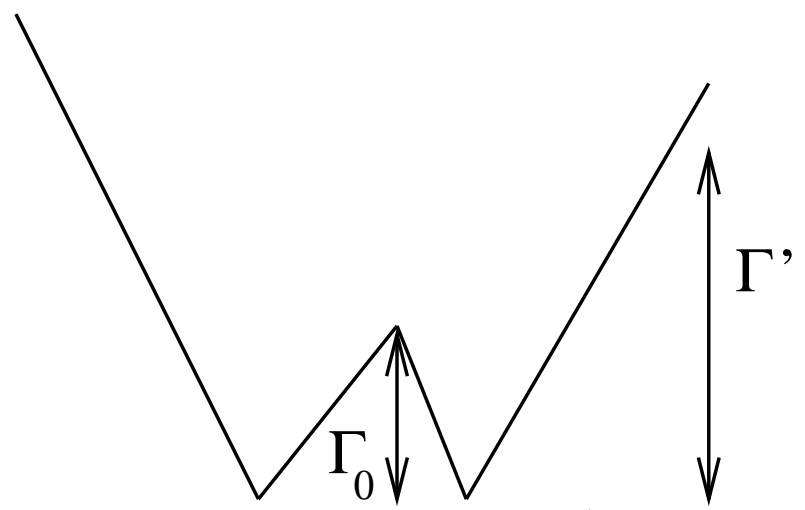

FIG. 6. Well with two almost degenerate minima (potential difference of order $O(1)$ and a barrier $\Gamma_{0}$ which contribute to the inwell equilibrium dynamics.

behavior will instead be dominated by rare configurations (but less rare than the previous ones) in which the valley at time $t^{\prime}$ has two almost degenerate minima, separated by a barrier $\Gamma_{0}$, as represented in Fig. 6. Jumping between such minima persists even for $t^{\prime} \rightarrow \infty$. The motion between these minima is equilibrium motion, since typically $\Gamma_{0}<\Gamma^{\prime}$ and the packet are already well equilibrated. In this limit 
the statistics of the infinitely deep valley potential becomes that of a random walk restricted to have $U_{i}-U_{\text {valley-min }}>0$ 11,16

Thus in this second regime $t-t^{\prime} \sim t^{\prime \alpha}$ with fixed $\alpha=\frac{\ln \left(t-t^{\prime}\right)}{\ln t^{\prime}}<1$ we will find that the diffusion front for relative displacements $Q\left(y, t, t^{\prime}\right)$ has, in addition to a $\delta$-function part for the rescaled variable $\tilde{y}=y / \ln ^{2}\left(t-t^{\prime}\right.$ ) (of weight almost equal to 1 which corresponds to the typical valleys), an additional-subdominant by $\frac{1}{\ln \left(t, t^{\prime}\right)}$ - smooth part:

$$
\tilde{Q}\left(y, t, t^{\prime}\right) \sim \frac{T}{\left(T \ln \left(t-t^{\prime}\right)\right)^{3}} f_{\alpha}\left[\frac{y}{\left(T \ln \left(t-t^{\prime}\right)\right)^{2}}\right]
$$

where the function $f_{\alpha}$ is universal. This result is obtained in section (VIF).

(ii) "crossover regime": $t \sim t^{\prime}, \alpha=1$ :

Finally, the matching between the regimes (i) and (ii) as $t-t^{\prime} \sim t^{\prime}$ is studied in Section VIG.

Before closing this section, it is interesting to note that by computing the twotime diffusion front, we obtain simultaneously the answer to the problem of evolution of two independent particles in the same environment, each seeing a thermal noise with a different temperature $T$, for the first particle with trajectory $x(t)$, and $T^{\prime}<T$ for the second with trajectory $x^{\prime}(t)$. If the two particles start from the same point 0 at $t=0$, it is clear from the considerations of the effective dynamics that the distribution of their respective rescaled positions $X=x /(\ln t)^{2}$ and $\tilde{X}^{\prime}=x^{\prime} /(\ln t)^{2}$ will be given by $P\left(x, x^{\prime}, t\right) \sim P_{\alpha=T / T^{\prime}}\left(X, \tilde{X}^{\prime}\right)$ with the same scaling function as in (128). [Although in the aging problem the thermal noises are identical between $t$

and $t^{\prime}$, this does not make a difference at large times for rescaled quantities.] Note that it should be easier to measure the dependence on $T$ rather than $\ln t$ as the latter in practice cannot be varied over a wide range.

\section{B. Singular part of the two time diffusion front: symmetric case}

\section{Probability $D_{\alpha}$ of staying within a well from $t^{\prime}$ to $t$}

We start by computing the probability for a particle to stay within the bottom of a valley between $t^{\prime}$ and $t$ :

$$
D_{\alpha}=\int_{-\infty}^{\infty} d \tilde{X}^{\prime} D_{\alpha}\left(\tilde{X}^{\prime}\right)
$$

We compute the fraction of walkers $D_{\Gamma, \Gamma^{\prime}}(\zeta)$ which are on a bond $F=\Gamma+\zeta$ at $\Gamma$ and have not moved from $\Gamma^{\prime}$ to $\Gamma$. This means that (i) this bond has not been decimated, (ii) one of its neighbors has not been decimated either (the neighbor in the same valley, i.e., the right neighbor for a descending bond and left neighbor for an ascending one). Thus the bond has been able to grow only on one side. As a result $D$ satisfies the RG equation:

$$
\left(\partial_{\Gamma}-\partial_{\zeta}\right) D_{\Gamma, \Gamma^{\prime}}(\zeta)=-P_{\Gamma}(0) D_{\Gamma, \Gamma^{\prime}}(\zeta)+P_{\Gamma}(0)\left(P_{\Gamma}(.) *_{\zeta} D_{\Gamma, \Gamma^{\prime}}(.)-D_{\Gamma, \Gamma^{\prime}}(\zeta)\right)
$$


The second and third terms on the r.h.s come from the allowed decimations of the neighbor. Integration over $\zeta$ shows the total loss of weight from the forbidden decimations: (ii) from the first term in the r.h.s. and (i) from the boundary term at $\zeta=0$. We are interested in large $\Gamma^{\prime}$, and thus we can assume that $P_{\Gamma}$ has reached its fixed point value (11). Thus one obtains, in the rescaled variables $\eta=\frac{\zeta}{\Gamma}$ and $\alpha=\frac{\Gamma}{\Gamma^{\prime}}$

$$
\left(\alpha \partial_{\alpha}-(1+\eta) \partial_{\eta}+1\right) D_{\alpha}(\eta)=\int_{0}^{\eta_{1}} e^{-\eta_{1}} D_{\alpha}\left(\eta-\eta_{1}\right)
$$

The initial condition $D_{\alpha=1}(\eta)$ corresponds to the probability that a walker be on a bond with $F=\Gamma^{\prime}(1+\eta)$ at $\Gamma^{\prime}$ and, since this probability is proportional to the length of the bond, it is obtained from (11) as

$$
D_{\alpha=1}(\eta)=\frac{1}{\bar{\lambda}} \int_{0}^{\infty} d \lambda \lambda P(\eta, \lambda)=\frac{1}{3}(1+2 \eta) e^{-\eta} .
$$

The solution of (135) reads:

$$
D_{\alpha}(\eta)=e^{-\eta} \frac{1}{3 \alpha^{2}}\left(5+\frac{2}{\alpha-1} e^{1-\alpha}\right)-e^{-\alpha \eta} \frac{2}{3(\alpha-1)} e^{1-\alpha} .
$$

We note that this barrier distribution is a combination of two exponential factors, the expected one $\exp (-F / \Gamma)$, and the other one $\exp \left(-F / \Gamma^{\prime}\right)$ which represents the "memory" from the condition at $t^{\prime}$. This will be the typical form for barrier distributions which we will encounter in all aging calculations.

We then obtain from (137) $D\left(t, t^{\prime}\right)=D_{\alpha}=\int_{0}^{\infty} d \eta D_{\alpha}(\eta)$, i.e., the probability that a walker has not moved from $t^{\prime}$ to $t$, which takes the remarkably simple universal form:

$$
D\left(t, t^{\prime}\right)=\left(\frac{\ln t^{\prime}}{\ln t}\right)^{2}\left(\frac{5}{3}-\frac{2}{3} e^{-\left(\frac{\ln t}{\ln t^{\prime}}-1\right)}\right) .
$$

The behavior of (138) for close times $\alpha=\frac{\ln t}{\ln t^{\prime}}$ near 1 is dominated by valleys about to be decimated at $\Gamma^{\prime}$. Expanding (138) yields $D\left(t, t^{\prime}\right) \sim 1-\frac{4}{3}(\alpha-1)$. The factor $4 / 3$ is consistent with the most probable number of jumps growing as $4 / 3 \ln t$ found earlier. Let us note that $-\partial_{t^{\prime}} D\left(t, t^{\prime}\right)$ with $D\left(t, t^{\prime}\right)$ given in (138), also represents the scaled distribution of the first passage time $t^{\prime}$ at the bottom of the renormalized valley where the particle is at $t$. This is consistent with the result of Golosov [27].

\section{Weight $D_{\alpha}\left(\tilde{X}^{\prime}\right)$ of the delta function component of $P_{\alpha}\left(X, \tilde{X}^{\prime}\right)$}

To compute the full singular part $D_{\alpha}\left(\tilde{X}^{\prime}\right)$ in (130) we simply have to extend the previous calculation keeping track of the length of the bond. Since we are not interested in the length at $\Gamma$ the length only appears at $\Gamma^{\prime}$ as a parameter in the initial condition. The final results reads: 


$$
\begin{aligned}
& D_{\alpha}\left(\tilde{X}^{\prime}\right)=\alpha^{2} d_{\alpha}\left(\alpha^{2}\left|\tilde{X}^{\prime}\right|\right) \\
& d_{\alpha}\left(X^{\prime}\right)=L T_{p^{\prime} \rightarrow\left|X^{\prime}\right|}^{-1} \frac{1}{\alpha^{2} p^{\prime}}\left(1-\frac{a\left(p^{\prime}\right)}{b^{2}\left(p^{\prime}\right)}+\frac{a\left(p^{\prime}\right)\left(1-b\left(p^{\prime}\right)\right)}{b^{2}\left(p^{\prime}\right)} e^{-(\alpha-1) b\left(p^{\prime}\right)}\right)
\end{aligned}
$$

One can check that for $\alpha=1$, one recovers the Laplace transform of the Kesten distribution (39), $\hat{d}_{1}\left(p^{\prime}\right)=\hat{q}\left(p^{\prime}\right)=\frac{1}{p^{\prime}}\left(1-\frac{a\left(p^{\prime}\right)}{b\left(p^{\prime}\right)}\right)$. Also one recovers $\hat{d}_{\alpha}\left(p^{\prime}=0\right)=\frac{1}{2} D_{t, t^{\prime}}$ with $D\left(t, t^{\prime}\right)$ given in (138) (with a factor $\frac{1}{2}$ corresponding to the total probability restricted to the half axis $\tilde{x}^{\prime}>0$ ).

This result (139) can be explicitly Laplace inverted in the limit of a large $\alpha=\frac{\ln t}{\ln t^{\prime}}$, where one can neglect the exponential term, yielding

$$
d_{\alpha}\left(X^{\prime}\right) \sim \frac{2}{\alpha^{2}} \int_{X^{\prime}}^{+\infty} d \lambda^{\prime} \lambda^{\prime} P\left(\lambda^{\prime}\right)=D_{\alpha} \Psi\left(X^{\prime}\right)
$$

where $D_{\alpha} \sim 5 /\left(3 \alpha^{2}\right)$ from $(\overline{138})$ is the total weight of particles remaining in their wells and $\Psi\left(X^{\prime}\right)$ is the normalized distribution of their positions:

$$
\Psi\left(X^{\prime}\right)=\frac{6}{5} \sum_{n=-\infty}^{+\infty} \frac{(-)^{n}}{\pi^{3}\left(n+\frac{1}{2}\right)^{3}}\left(1+\pi^{2}\left(n+\frac{1}{2}\right)^{2}\left|X^{\prime}\right|\right) e^{-\pi^{2}\left(n+\frac{1}{2}\right)^{2}\left|X^{\prime}\right|}
$$

We note that compared to the Kesten distribution $q\left(X^{\prime}=x^{\prime} / \Gamma^{\prime 2}\right), \Psi\left(X^{\prime}=x^{\prime} / \Gamma^{2}\right)$ has more weight towards the larger values of $x^{\prime} / \Gamma^{\prime 2}$. This is a consequence of the fact that the farther the particle goes the more likely it is to be in a deep well where it is likely to remain longer without further motion.

Finally, it is instructive to estimate also the singular part of the averaged conditional probability $\overline{\operatorname{Prob}\left(x t \mid x^{\prime} t^{\prime}, 00\right)}$. Using a similar method it is found to be:

$$
\hat{D}_{\alpha}^{\text {cond }}\left(X^{\prime}\right)=\frac{d_{\alpha}\left(X^{\prime}\right)}{q\left(X^{\prime}\right)}
$$

where $d_{\alpha}\left(X^{\prime}\right)$ is the function defined in (139).

\section{Probability of staying within a well: biased case}

Next we obtain the probability $D\left(t, t^{\prime}\right)$ that a walker does not move substantially between $t$ and $t^{\prime}$ (i.e., does not jump to a new valley bottom) in the presence of a small bias. This can be computed by extending to the biased case the direct method of section VIB, or from the more general approach presented in the next section. Here we only quote the end result:

$D_{\Gamma, \Gamma^{\prime}}=\frac{1}{\sinh ^{2} \gamma}\left[\left(2 \sinh ^{2} \gamma^{\prime}+1-\gamma^{\prime} \operatorname{coth} \gamma^{\prime}\right)-e^{-\left(\gamma-\gamma^{\prime}\right) \operatorname{coth} \gamma^{\prime}} \cosh \gamma\left(\cosh \gamma^{\prime}-\frac{\gamma^{\prime}}{\sinh \gamma^{\prime}}\right)\right]$

where

$$
\gamma \equiv \delta T \ln t \quad \text { and } \quad \gamma^{\prime} \equiv \delta T \ln t^{\prime}
$$

This formula is exact in the small bias scaling limit $t, t^{\prime} \rightarrow \infty, \delta \rightarrow 0$, with fixed $\gamma$ and $\gamma^{\prime}$. 
This formula (143) is interesting as it exhibits a crossover between two different aging scaling functions corresponding to the symmetric model and the directed model, respectively. In the limit $\delta \rightarrow 0$, i.e., $\gamma \rightarrow 0, \gamma^{\prime} \rightarrow 0$ with a fixed ratio $\gamma / \gamma^{\prime}=\alpha=\frac{\ln t}{\ln t^{\prime}}$ Eq. (138) is recovered. In the opposite limit in which both $\gamma \gg 1$ and $\gamma^{\prime} \gg 1$, a nontrivial scaling limit exists when $\gamma-\gamma^{\prime}$ is kept fixed, i.e., $t / t^{\prime}$ fixed, and the above expression simplifies to:

$$
D\left(t, t^{\prime}\right) \sim e^{-2\left(\gamma-\gamma^{\prime}\right)}=\frac{L\left(t^{\prime}\right)}{L(t)} \quad \text { with } \quad L(t)=\bar{l}_{\Gamma} \sim t^{\mu}
$$

using $\mu=2 \delta T$. This coincides with the small $\mu$ limit of the aging form of the directed model (formula (51) of [16]) which can be written as $D\left(t, t^{\prime}\right)=H\left[\left(t^{\prime} / t\right)^{\mu}\right]$ with $H[z]=\left(\frac{\sin (\pi \mu)}{\mu \pi}\right) \int_{0}^{z} d y\left(1-y^{1 / \mu}\right)^{-\mu}$. When $\mu \rightarrow 0$ the function $H$ becomes exactly $H[z]=z$, and one recovers (144).

\section{Two-time diffusion front: full analysis}

\section{Sketch of the method}

To compute the two time diffusion front $\overline{\operatorname{Prob}\left(x t, x^{\prime} t^{\prime} \mid 00\right)}$ in the general biased case, we need to introduce quantities associated with bonds which keep track of their endpoints and for which a RG equation can be written. We thus define $\Omega_{\Gamma, \Gamma^{\prime}}^{++}\left(\zeta, x_{L}, x_{R} ; x_{L}^{\prime}, x_{R}^{\prime}\right)$ (resp. $\Omega_{\Gamma, \Gamma^{\prime}}^{-+}\left(\zeta, x_{L}, x_{R} ; x_{L}^{\prime}, x_{R}^{\prime}\right)$ ) as the probability that the origin belongs to a descending bond with ends $\left[-x_{L}^{\prime}, x_{R}^{\prime}\right]$ at $\Gamma^{\prime}$ and to a descending (resp. ascending) bond of barrier $\zeta=F-\Gamma$ and with ends $\left[-x_{L}, x_{R}\right]$ at $\Gamma$. Similar definitions hold with $\Omega^{+-}$and $\Omega^{--}$for an ascending bond at $\Gamma^{\prime}$. From these quantities one can recover the two-time diffusion front for $x^{\prime}>0$ :

$$
\begin{aligned}
& \overline{\operatorname{Prob}\left(x, t, x^{\prime}>0, t^{\prime} \mid 0,0\right)}=\theta(x) \int_{0}^{\infty} d \zeta \int_{0}^{\infty} d x_{L} \int_{0}^{\infty} d x_{L}^{\prime} \Omega_{\Gamma, \Gamma^{\prime}}^{++}\left(\zeta, x_{L}, x_{R}=x ; x_{L}^{\prime}, x_{R}^{\prime}=x^{\prime}\right) \\
& +\theta(-x) \int_{0}^{\infty} d \zeta \int_{0}^{\infty} d x_{R} \int_{0}^{\infty} d x_{L}^{\prime} \Omega_{\Gamma, \Gamma^{\prime}}^{-+}\left(\zeta, x_{L}=-x, x_{R} ; x_{L}^{\prime}, x_{R}^{\prime}=x^{\prime}\right)
\end{aligned}
$$

and similarly for $x^{\prime}<0$ :

$$
\begin{aligned}
& \overline{\operatorname{Prob}\left(x, t, x^{\prime}<0, t^{\prime} \mid 0,0\right)}=\theta(x) \int_{0}^{\infty} d \zeta \int_{0}^{\infty} d x_{L} \int_{0}^{\infty} d x_{R}^{\prime} \Omega_{\Gamma, \Gamma^{\prime}}^{+-}\left(\zeta, x_{L}, x_{R}=x ; x_{L}^{\prime}=-x^{\prime}, x_{R}^{\prime}\right) \\
& +\theta(-x) \int_{0}^{\infty} d \zeta \int_{0}^{\infty} d x_{R} \int_{0}^{\infty} d x_{R}^{\prime} \Omega_{\Gamma, \Gamma^{\prime}}^{--}\left(\zeta, x_{L}=-x, x_{R} ; x_{L}^{\prime}=-x^{\prime}, x_{R}^{\prime}\right)
\end{aligned}
$$

The four RG equations for the four quantities $\Omega_{\Gamma, \Gamma^{\prime}}^{+\epsilon^{\prime}}\left(\zeta, x_{L}, x_{R} ; x_{L}^{\prime}, x_{R}^{\prime}\right)$ and $\Omega_{\Gamma, \Gamma^{\prime}}^{-\epsilon^{\prime}}\left(\zeta, x_{L}, x_{R} ; x_{L}^{\prime}, x_{R}^{\prime}\right)$, with $\epsilon^{\prime}= \pm 1$ can be written in a compact form:

$$
\begin{aligned}
& \left(\partial_{\Gamma}-\partial_{\zeta}\right) \Omega_{\Gamma, \Gamma^{\prime}}^{ \pm \epsilon^{\prime}}\left(\zeta, x_{L}, x_{R} ; x_{L}^{\prime}, x_{R}^{\prime}\right)=-2 P_{\Gamma}^{\mp}(0) \Omega_{\Gamma, \Gamma^{\prime}}^{ \pm \epsilon^{\prime}}\left(\zeta, x_{L}, x_{R} ; x_{L}^{\prime}, x_{R}^{\prime}\right) \\
& +\int_{l_{1}>0, l_{2}>0, y>0} P_{\Gamma}^{\mp}\left(0, l_{2}\right) P_{\Gamma}^{ \pm}\left(., l_{1}\right) *_{\zeta} \Omega_{\Gamma, \Gamma^{\prime}}^{ \pm \epsilon^{\prime}}\left(., y, x_{R} ; x_{L}^{\prime}, x_{R}^{\prime}\right) \delta\left(x_{L}-\left(y+l_{1}+l_{2}\right)\right) \\
& +\int_{l_{2}>0, l_{3}>0, y>0} P_{\Gamma}^{\mp}\left(0, l_{2}\right) P_{\Gamma}^{ \pm}\left(., l_{3}\right) *_{\zeta} \Omega_{\Gamma, \Gamma^{\prime}}^{ \pm \epsilon^{\prime}}\left(., x_{L}, y ; x_{L}^{\prime}, x_{R}^{\prime}\right) \delta\left(x_{R}-\left(y+l_{2}+l_{3}\right)\right)
\end{aligned}
$$




$$
\begin{aligned}
& +\int_{l_{1}>0, l_{3}>0, y_{1}>0, y_{2}>0} P_{\Gamma}^{ \pm}\left(., l_{1}\right) *_{\zeta} P_{\Gamma}^{ \pm}\left(., l_{3}\right) \Omega_{\Gamma, \Gamma^{\prime}}^{\mp \epsilon^{\prime}}\left(0, y_{1}, y_{2} ; x_{L}^{\prime}, x_{R}^{\prime}\right) \\
& \quad \delta\left(x_{L}-\left(y_{1}+l_{1}\right)\right) \delta\left(x_{R}-\left(y_{2}+l_{3}\right)\right)
\end{aligned}
$$

These equations must be solved with the following initial conditions at $\Gamma=\Gamma^{\prime}$ :

$$
\Omega_{\Gamma^{\prime}, \Gamma^{\prime}}^{\epsilon \epsilon^{\prime}}\left(\zeta, x_{L}, x_{R} ; x_{L}^{\prime}, x_{R}^{\prime}\right)=\delta_{\epsilon, \epsilon^{\prime}} \delta\left(x_{L}-x_{L}^{\prime}\right) \delta\left(x_{R}-x_{R}^{\prime}\right) \omega_{\Gamma^{\prime}}^{\epsilon^{\prime}}\left(\zeta, x_{L}^{\prime}, x_{R}^{\prime}\right)
$$

where $\omega_{\Gamma^{\prime}}^{\epsilon^{\prime}}\left(\zeta, x_{L}^{\prime}, x_{R}^{\prime}\right)$ is the probability that the origin belongs at $\Gamma^{\prime}$ to a bond (ascending if $\epsilon^{\prime}=-1$ and descending if $\epsilon^{\prime}=+1$ ) with barrier $\zeta=F-\Gamma^{\prime}$ and of ends $\left[-x_{L}^{\prime}, x_{R}^{\prime}\right]$ at $\Gamma^{\prime}$ :

$$
\omega_{\Gamma^{\prime}}^{\epsilon^{\prime}}\left(\zeta, x_{L}^{\prime}, x_{R}^{\prime}\right)=\int_{0}^{\infty} d l^{\prime} \frac{P_{\Gamma^{\prime}}^{\epsilon^{\prime}}\left(\zeta, l^{\prime}\right)}{\bar{l}_{\Gamma^{\prime}}} \delta\left(l^{\prime}-\left(x_{L}^{\prime}+x_{R}^{\prime}\right)\right)=\frac{1}{\bar{l}_{\Gamma^{\prime}}} P_{\Gamma^{\prime}}^{\epsilon^{\prime}}\left(\zeta, x_{L}^{\prime}+x_{R}^{\prime}\right)
$$

Note that all the primed quantities - those at the earlier time enter only via the initial conditions on the $\Omega$ 's. These equations (147), together with the initial condition (148) are solved explicitly using Laplace transforms in Appendix F. For the symmetric case the explicit expression for the Laplace transform of the full distribution $\overline{\operatorname{Prob}\left(x t, x^{\prime} t^{\prime} \mid 00\right)}$ with respect to $x$ and $x^{\prime}$ is given in (F41,F42). In the next two subsections we give explicit expressions for some simpler quantities.

\section{Some results for the symmetric case}

We first give the explicit expression for the distribution $Q\left(y, t, t^{\prime}\right)$ of relative displacements $y=x(t)-x\left(t^{\prime}\right)$, with $Q\left(y, t, t^{\prime}\right)=\int d x^{\prime} \overline{P\left(x^{\prime}+y, t, x^{\prime}, t^{\prime} \mid 00\right)}$. This

distribution takes the scaling form:

$$
Q\left(y, t, t^{\prime}\right) \sim \frac{1}{\left(T \ln t^{\prime}\right)^{2}} Q_{\alpha=\frac{\ln t}{\ln t^{\prime}}}\left(Y=\frac{|y|}{\left(T \ln t^{\prime}\right)^{2}}\right) .
$$

Note that we have chosen $Y=\frac{y}{\Gamma^{\prime 2}}$ as the scaling variable here for convenience.

From Appendix $\mathbb{E}$ the Laplace transform $\hat{Q}_{\alpha}(p)=\int_{0}^{\infty} d Y e^{-p Y} Q_{\alpha}(Y)$ is found to be:

$$
\begin{aligned}
\hat{Q}_{\alpha}(p) & =\frac{\tanh (\sqrt{p} \alpha)}{p \alpha^{2}}\left(-\frac{1}{2 \sqrt{p}}-\sqrt{p}+\left(\frac{5 p}{6}+\frac{1}{2}\right) \operatorname{coth} \sqrt{p}+\sinh \sqrt{p}\left(\cosh \sqrt{p}-\frac{\sinh \sqrt{p}}{\sqrt{p}}\right)\right) \\
& +\frac{\cosh \sqrt{p}}{p \alpha^{2}}\left(\frac{\sqrt{p}}{\sinh \sqrt{p}}+\frac{\sinh \sqrt{p}}{\sqrt{p}}-\cosh \sqrt{p}\right) \\
& -\frac{1}{6 p \alpha^{2} \cosh (\sqrt{p} \alpha)}\left(\frac{3}{\cosh \sqrt{p}}+\frac{\sqrt{p}}{\sinh \sqrt{p}} \frac{p-3}{p-1}\right) \\
& +e^{-(\alpha-1)} \frac{(1-\sqrt{p} \operatorname{coth} \sqrt{p})(1+\sqrt{p} \tanh (\sqrt{p} \alpha))}{3 \alpha^{2}(p-1)} \\
& +\frac{e^{-(\alpha-1) \sqrt{p} \operatorname{coth} \sqrt{p}}}{2 p \alpha^{2} \cosh (\sqrt{p} \alpha)}\left(\cosh \sqrt{p}+\frac{1}{\cosh \sqrt{p}}-\frac{\sqrt{p}}{\sinh \sqrt{p}}-\frac{\sinh \sqrt{p}}{\sqrt{p}}\right)
\end{aligned}
$$

Several properties of this expression can be checked explicitly. First, from normalization on the half space $Y>0$ one has $\hat{Q}_{\alpha}(p=0)=\frac{1}{2}$. Then, the initial condition 
at $t=t^{\prime}$ is $\hat{Q}_{\alpha=1}(p)=\frac{1}{2}$ since $Q\left(y, t, t^{\prime}\right)=\delta(y)$. One can also recover the singular part $D\left(t, t^{\prime}\right) \delta(y)$ of the distribution corresponding to walkers which have not moved appreciably between $t^{\prime}$ and $t$. Indeed one finds $\hat{Q}_{\alpha}(p \rightarrow+\infty) \sim D_{\alpha} / 2$ where $D_{\alpha}$ is given by (138). Finally, for very separated times $\alpha \rightarrow+\infty$ one recovers the Kesten distribution (39) since the initial condition at $\Gamma^{\prime}$ has been forgotten:

$$
\hat{Q}_{\alpha}\left(p=\frac{k}{\alpha^{2}}\right) \rightarrow \frac{1}{k}\left(1-\frac{1}{\cosh \sqrt{k}}\right) .
$$

From the above result (151) one obtains the moments of the relative displacements which take the general scaling form:

$$
\begin{aligned}
& \overline{\left\langle\left|x(t)-x\left(t^{\prime}\right)\right|^{n}\right\rangle} \sim\left(T \ln t^{\prime}\right)^{2 n} F_{n}\left[\frac{\ln t}{\ln t^{\prime}}\right] \\
& F_{n}[\alpha]=a_{n}(\alpha)+e^{1-\alpha} b_{n}(\alpha) .
\end{aligned}
$$

We give explicitly the form of the second moment:

$$
\begin{aligned}
& a_{2}(\alpha)=\frac{61 \alpha^{4}}{180}-\frac{4 \alpha}{5}+\frac{47}{60}+\frac{2}{7 \alpha}-\frac{409}{378 \alpha^{2}} \\
& b_{2}(\alpha)=-\frac{2}{9}+\frac{8}{27 \alpha}+\frac{2}{5 \alpha^{2}}
\end{aligned}
$$

while the first moment has a simpler expression:

$$
\overline{\left\langle\left|x(t)-x\left(t^{\prime}\right)\right|\right\rangle} \sim\left(T \ln t^{\prime}\right)^{2}\left(\frac{5}{12} \alpha^{2}-\frac{6}{5 \alpha}+\frac{221}{180 \alpha^{2}}-\frac{4}{9 \alpha^{2}} e^{-(\alpha-1)}\right) .
$$

At large $\alpha$ one recovers Sinai's result (from (39)). When $t$ and $t^{\prime}$ are not too separated, i.e., $\alpha \approx 1$ one finds:

$$
F_{2}[\alpha] \sim \frac{272}{315}(\alpha-1)
$$

and the motion is much slower consistent with the aging of the system.

Again it is simple to recover the behavior for relatively close times $\alpha \approx 1$. Expanding the above (151) one finds:

$$
\begin{aligned}
& 2 \hat{Q}_{\alpha}(p) \sim 1-\frac{4}{3}(\alpha-1)+(\alpha-1) \mathcal{H}(p)+O\left((\alpha-1)^{2}\right) \\
& \mathcal{H}(p)=\frac{1}{\cosh \sqrt{p} \sinh \sqrt{p}}\left(\sqrt{p}-\frac{1}{\sqrt{p}}\right)+\frac{1}{(\sinh \sqrt{p})^{2}}
\end{aligned}
$$

The $1-4(\alpha-1) / 3, p$-independent term represents the probability that the particule does not jump and has been discussed in Section $\nabla T \mathrm{~B}$. The other term $\mathcal{H}(p)$ is the Laplace transform of the probability that the jump is over a scaled distance $\lambda=Y, \mathcal{H}(\lambda)=\int d \lambda_{1}\left(1+\frac{\lambda_{1}}{\bar{\lambda}}\right) P\left(0, \lambda_{1}\right) P\left(\lambda-\lambda_{1}\right)$ where the first term corresponds to the bond containing the origin being decimated and the second term corresponds to the neighboring bond in the same valley being decimated. In Laplace variables this gives $\mathcal{H}(p)=\left(\left(-2 \partial_{p} P(\eta=0, p)+P(\eta=0, p)\right) P(p)\right)$ which using (11) gives back the above result (158). 
We also give the explicit expression for the normalized dimensionless correlation:

$$
\begin{aligned}
& \frac{\overline{\left\langle x(t) x\left(t^{\prime}\right)\right\rangle}}{\sqrt{\overline{\left\langle x^{2}(t)\right\rangle}} \sqrt{\overline{\left\langle x^{2}\left(t^{\prime}\right)\right\rangle}}}=\frac{72}{61 \alpha}-\frac{40}{61 \alpha^{2}}-\frac{180}{427 \alpha^{3}}+\frac{2045}{1281 \alpha^{4}} \\
& +e^{-(\alpha-1)}\left(\frac{20}{61 \alpha^{2}}-\frac{80}{183 \alpha^{3}}-\frac{36}{61 \alpha^{4}}\right)
\end{aligned}
$$

which decreases from 1 to 0 as $\alpha=\frac{\ln t}{\ln t^{\prime}}$ goes from 1 to $+\infty$. Note that the decay as $1 / \alpha$ for large $\alpha$ is characteristic of the generic decay of corrections to asymptotics as $1 / \Gamma$.

\section{Some results for the biased case}

From Appendix $\mathrm{F}$ one has in principle an exact expression for the Laplace transformed two-time diffusion front. It is, however, very complicated and thus we give here only a few simpler quantities.

Given that the bias is towards $x>0$ and that the starting point is $x=0$, the probability that the particle is on the side $x>0$ both at $t^{\prime}$ and $t$ has the aging behavior:

$$
\begin{aligned}
P^{++}\left(\Gamma, \Gamma^{\prime}\right) & =\overline{\left\langle\theta(x(t)) \theta\left(x\left(t^{\prime}\right)\right)\right\rangle}=\frac{1}{16 \sinh ^{2} \gamma \sinh ^{2} \gamma^{\prime}}\left(e^{2 \gamma}\left(e^{2 \gamma^{\prime}}-2 \gamma^{\prime}-1\right)\right. \\
& \left.-2 \gamma\left(e^{-2 \gamma^{\prime}}+2 \gamma^{\prime}-1\right)-2 e^{2 \gamma^{\prime}}-e^{-2 \gamma^{\prime}}+4 \gamma^{\prime 2}+2 \gamma^{\prime}+3\right)
\end{aligned}
$$

with $\gamma=\Gamma \delta$ and $\gamma^{\prime}=\Gamma^{\prime} \delta$, while $P^{--}\left(\Gamma, \Gamma^{\prime}\right)$ is obtained by $\delta \rightarrow-\delta$. The other possibilities, $P^{+-,-+}\left(\Gamma, \Gamma^{\prime}\right)$ are obtained from the single time identities $P^{++}\left(\Gamma, \Gamma^{\prime}\right)+$ $P^{+-}\left(\Gamma, \Gamma^{\prime}\right)=P^{+}(\Gamma)=\bar{l}_{\Gamma}^{+} / \bar{l}_{\Gamma}$ and $P^{++}\left(\Gamma, \Gamma^{\prime}\right)+P^{-+}\left(\Gamma, \Gamma^{\prime}\right)=P^{+}\left(\Gamma^{\prime}\right)=\bar{l}_{\Gamma^{\prime}}^{+} / \bar{l}_{\Gamma^{\prime}}$ where $\bar{l}_{\Gamma^{\prime}}^{ \pm}$are given in $(24)$

The explicit expression for the correlation $\left\langle x(t) x\left(t^{\prime}\right)\right\rangle$ is given by (F43).

\section{E. Full two-time aging function in a semi-infinite system}

There is one instructive situation where it is simpler to obtain explicitly the full two-time probability distribution $\overline{\operatorname{Prob}\left(x t, x^{\prime} t^{\prime} \mid 00\right)}$, even in the presence of a bias. This is the case of Sinai diffusion on a semi-infinite axis $(0,+\infty)$ with a reflecting boundary at $x=0$ and possibly some drift in the $(+)$ direction.

In this case the single time diffusion front is simply $\overline{\operatorname{Prob}_{R}^{+}\left(x^{\prime} t^{\prime} \mid 00\right)}=E_{\Gamma^{\prime}}^{+}\left(x^{\prime}\right)$, i.e., the probability distribution of the length $x^{\prime}$ of the first renormalized bond near the boundary, whose Laplace transform is given in equation (35). Similarly, the twotime diffusion front is equal to $\overline{\operatorname{Prob}_{R}^{+}\left(x t, x^{\prime} t^{\prime} \mid 00\right)}=E_{\Gamma, \Gamma^{\prime}}^{+}\left(x, x^{\prime}\right)$, i.e., the probability that the first bond near the boundary has length $x^{\prime}$ at $\Gamma^{\prime}$ and length $x$ at $\Gamma$. Its RG equation is given by (10)

$$
\partial_{\Gamma} E_{\Gamma, \Gamma^{\prime}}^{+}\left(x, x^{\prime}\right)=P_{\Gamma}^{-}(0, .) *_{x} E_{\Gamma, \Gamma^{\prime}}^{+}\left(., x^{\prime}\right) *_{x} \int_{0}^{\infty} d \zeta^{\prime} P_{\Gamma}^{+}\left(\zeta^{\prime}, .\right)-E_{\Gamma, \Gamma^{\prime}}^{+}\left(x, x^{\prime}\right) \int_{0}^{\infty} d l^{\prime} P_{\Gamma}^{-}\left(0, l^{\prime}\right)
$$


but with the initial condition

$$
E_{\Gamma^{\prime}, \Gamma^{\prime}}^{+}\left(x, x^{\prime}\right)=\delta\left(x-x^{\prime}\right) E_{\Gamma^{\prime}}^{+}\left(x^{\prime}\right)
$$

at $\Gamma=\Gamma^{\prime}$. Note that within the effective dynamics this system has the flavor of a directed model, since $x(t)-x\left(t^{\prime}\right)$ is always positive. It is thus convenient to define the double Laplace transform $\hat{E}_{\Gamma, \Gamma^{\prime}}^{+}\left(p, p^{\prime}\right)=\int_{0}^{+\infty} d x^{\prime} e^{-p^{\prime} x^{\prime}} \int_{x^{\prime}}^{\infty} d x e^{-p\left(x-x^{\prime}\right)} E_{\Gamma, \Gamma^{\prime}}^{+}\left(x, x^{\prime}\right)$ and using the fixed point solution (22) for $P_{\Gamma}^{ \pm}$together with the properties (20) the above RG equation simplifies into $\partial_{\Gamma} \ln \hat{E}_{\Gamma, \Gamma^{\prime}}^{+}\left(p, p^{\prime}\right)=\partial_{\Gamma} \ln \frac{u_{\Gamma}^{+}(0)}{u_{\Gamma}^{+}(p)}$. Using the above initial condition, we obtain the factorized form $\hat{E}_{\Gamma, \Gamma^{\prime}}^{+}\left(p, p^{\prime}\right)=E_{\Gamma^{\prime}}^{+}\left(p^{\prime}\right) Q_{\Gamma, \Gamma^{\prime}}^{+}(p)$ i.e., in real space:

$$
\overline{\operatorname{Prob}_{R}^{+}\left(x t, x^{\prime} t^{\prime} \mid 00\right)}=E_{\Gamma^{\prime}}^{+}\left(x^{\prime}\right) Q_{\Gamma, \Gamma^{\prime}}^{+}\left(x-x^{\prime}\right)
$$

where $E_{\Gamma^{\prime}}^{+}\left(x^{\prime}\right)$ is by $(122)$ and the distribution of the relative displacements $y=$ $x(t)-x\left(t^{\prime}\right)$ is

$$
Q_{\Gamma, \Gamma^{\prime}}^{+}(y)=L T_{p \rightarrow y}^{-1} e^{-\delta\left(\Gamma-\Gamma^{\prime}\right)} \frac{\sinh \left(\delta \Gamma^{\prime}\right)}{\sinh (\delta \Gamma)} \frac{\left.\sqrt{p+\delta^{2}} \operatorname{coth}\left(\Gamma^{\prime} \sqrt{p+\delta^{2}}\right)-\delta\right)}{\sqrt{p+\delta^{2}} \operatorname{coth}\left(\Gamma \sqrt{p+\delta^{2}}\right)-\delta}
$$

In the symmetric case $\delta=0$, Laplace inversion gives the two-time front:

$$
\begin{aligned}
Q\left(y, t, t^{\prime}\right) & =\frac{2}{\Gamma^{2}} \sum_{n=0}^{\infty} \pi\left(n+\frac{1}{2}\right) \frac{\Gamma^{\prime}}{\Gamma} \operatorname{cotan}\left(\pi\left(n+\frac{1}{2}\right) \frac{\Gamma^{\prime}}{\Gamma}\right) e^{-\frac{y^{2}}{\Gamma^{2}} \pi^{2}\left(n+\frac{1}{2}\right)^{2}} \\
& +\frac{\Gamma^{\prime}}{\Gamma}\left(\delta(y)-\frac{2}{\Gamma^{\prime 2}} \sum_{m=1}^{\infty} \pi m \tan \left(\pi m \frac{\Gamma}{\Gamma^{\prime}}\right) e^{-\frac{y^{2}}{\Gamma^{\prime 2}} \pi^{2} m^{2}}\right)
\end{aligned}
$$

with $\Gamma=T \ln t$ and $\Gamma^{\prime}=T \ln t^{\prime}$.

In the biased case one finds:

$$
\begin{aligned}
Q_{\Gamma, \Gamma^{\prime}}^{+}(y) & =\frac{e^{\gamma^{\prime}} \sinh \left(\gamma^{\prime}\right)}{e^{\gamma} \sinh (\gamma)}\left[\frac{2}{\Gamma^{2}} \sum_{n=0}^{+\infty} d_{n}\left(\gamma, \gamma^{\prime}\right) e^{-\frac{y}{\Gamma^{2}} s_{n}^{+}(\gamma)}\right. \\
& \left.+\delta(y)-\frac{2}{\Gamma^{\prime 2}} \sum_{m=1}^{+\infty} \frac{\pi^{2} m^{2}}{\pi m \operatorname{cotan}\left(\pi m \frac{\gamma}{\gamma^{\prime}}\right)-\gamma^{\prime}} e^{-\frac{y}{\Gamma^{\prime 2}}\left(\gamma^{\prime 2}+m^{2} \pi^{2}\right)}\right]
\end{aligned}
$$

where the $s_{n}^{+}(\gamma)$ are given in (50) and the $d_{n}\left(\gamma, \gamma^{\prime}\right)$ are given in terms of the $\alpha_{n}^{+}(\gamma)$ defined in (48) via

$$
d_{n}\left(\gamma, \gamma^{\prime}\right)=\alpha_{n}^{+}(\gamma)^{2} \frac{\alpha_{n}^{+}(\gamma) \operatorname{cotan}\left(\frac{\gamma^{\prime}}{\gamma} \alpha_{n}^{+}(\gamma)\right)-\gamma}{\alpha_{n}^{+}(\gamma)^{2}+\gamma^{2}-\gamma}
$$

except for the term $n=0$ in the domain $\gamma>1$ where

$$
d_{0}\left(\gamma, \gamma^{\prime}\right)=\tilde{\alpha}_{0}^{+}(\gamma)^{2} \frac{\tilde{\alpha}_{0}^{+}(\gamma) \operatorname{coth}\left(\frac{\gamma^{\prime}}{\gamma} \tilde{\alpha}_{0}^{+}(\gamma)\right)-\gamma}{\tilde{\alpha}_{0}^{+}(\gamma)^{2}-\gamma^{2}+\gamma}
$$


with $\tilde{\alpha}_{0}^{+}$given in (48). In the limit $\gamma=\Gamma \delta=T \delta \ln t \gg 1 \gamma^{\prime}=\Gamma^{\prime} \delta=T \delta \ln t^{\prime} \gg 1$, one finds the simple aging form:

$$
Q_{\Gamma, \Gamma^{\prime}}^{+}(y) \sim \frac{t^{\prime \mu}}{t^{\mu}} \delta(y)+\left(1-\frac{t^{\prime \mu}}{t^{\mu}}\right) \frac{\mu^{2}}{T^{2} t^{\mu}} e^{-y \mu^{2} /\left(T^{2} t^{\mu}\right)}
$$

with $\mu=2 \delta T$. Equation (168) coincides exactly with the small $\mu$ limit of the twotime diffusion front in the directed model [45]. As noted above the model with a reflecting wall is in effect asymptotically to a directed model. In the presence of a bias, in the long-time limit $\gamma, \gamma^{\prime} \gg 1$ it gives the same results as the full model discussed in the previous section, as the influence of the wall vanishes in that limit.

\section{F. Dynamics within a well}

We now study the dynamics in the time regime $t-t^{\prime} \sim t^{\prime \alpha}$ with fixed $\alpha<1$. As was discussed in VIA this is dominated by renormalized valleys at $\Gamma^{\prime}$ with two degenerate minima $U_{1}$ and $U_{2}$ with $U_{1}-U_{2}$ of order $O(T)$ as in Fig. 6. In a typical valley many such degeneracies may exist on small scales $y=x-x^{\prime}$ of order 1 , with non-universal statistics, but we are interested in rare valleys where such degeneracies exist on scales $y \sim \Gamma^{\prime 2}$ with barriers $\Gamma_{0} \sim \Gamma^{\prime}$; the distribution of these is universal.

We introduce the probability density $R_{\Gamma}\left(\zeta, l, x, \Gamma_{0}\right)$ that a renormalized bond at scale $\Gamma$ has length $l$, barrier $\zeta=F-\Gamma$ and has a secondary minimum, degenerate in energy with the absolute minimum (i.e., the lower edge of the bond) and separated from it by a distance $x$ and a barrier $\Gamma_{0}$. The calculation of this quantity is performed in Appendix E. We find the simple decoupled form:

$$
R_{\Gamma}\left(\zeta, l, x, \Gamma_{0}\right)=\theta\left(\Gamma-\Gamma_{0}\right) \theta(l-x) P_{\Gamma}(\zeta, l-x) \frac{1}{\Gamma_{0}^{4}} \hat{r}\left(x / \Gamma_{0}^{2}\right)
$$

with

$$
\hat{r}(X)=4 \sum_{n=1}^{\infty} n^{2} \pi^{2}\left(2 X n^{2} \pi^{2}-3\right) e^{-X n^{2} \pi^{2}} .
$$

We have written for simplicity $R$ in unrescaled variables, but the expression is of course valid only in the scaling regime $\zeta \sim \Gamma_{0} \sim \Gamma, x \sim l \sim \Gamma^{2}$ (see Appendix for details). Note that its total normalization is $\int_{0}^{+\infty} d \zeta \int_{0}^{\Gamma} d \Gamma_{0} \int_{0}^{+\infty} d l \int_{0}^{l} d x R_{\Gamma}\left(x, \Gamma_{0}\right) \sim$ $1 / \Gamma$ as expected since it corresponds to a rare event; with $T \ll \Gamma$, the density with double minima within $T$ of each other is $T R$.

We can now obtain the probability that a Sinai walker will move by $y$ between $t^{\prime}$ and $t$ for $t-t^{\prime} \sim t^{\prime \alpha}$ with $\alpha<1$. We need first the probability $K_{\Gamma^{\prime}}\left(y, \Gamma_{0}\right)$ that the the starting point happens to belong to a renormalized valley at $\Gamma^{\prime}=T \ln t^{\prime}$ which possesses two degenerate minima separated by a distance $y$ and a barrier $\Gamma_{0}<\Gamma^{\prime}$. Taking into account that each of the two bonds forming the valley may be the one with the degenerate minima (the probability that both have degenerate minima is negligible in the scaling regime of interest) one gets, using (170)

$$
\begin{aligned}
K_{\Gamma^{\prime}}\left(y, \Gamma_{0}\right) & =\frac{1}{\Gamma^{\prime 2}} \int_{l_{1}, l_{2}}\left(l_{1}+l_{2}\right)\left[P_{\Gamma^{\prime}}\left(l_{1}\right) R_{\Gamma^{\prime}}\left(l_{2}, y, \Gamma_{0}\right)+P_{\Gamma^{\prime}}\left(l_{2}\right) R_{\Gamma^{\prime}}\left(l_{1}, y, \Gamma_{0}\right)\right] \\
& =2\left(1+\frac{y}{\Gamma^{\prime 2}}\right) \frac{1}{\Gamma_{0}^{4}} \hat{r}\left(\frac{y}{\Gamma_{0}^{2}}\right)
\end{aligned}
$$


When the starting point belongs to such a valley (characterized by $y$ and $\Gamma_{0}$ ) the walker at $\Gamma^{\prime}$ is well equilibrated (since $\Gamma_{0}<\Gamma^{\prime}$ ) and its thermal distribution is, in a scaling sense, a sum of two delta function peaks separated by a distance $y>0$ with weights $p=1 /\left(1+e^{-w / T}\right.$ ) (at the abolute minimum) and $1-p$ (at the secondary minimum), where $w$ represents the (free) energy difference (of order $O(T)$ ) between the two minima. When estimating the distribution of $y=\left|x(t)-x\left(t^{\prime}\right)\right|$ one probes this equilibrium distribution at $t$ and $t^{\prime}$. Denoting $\hat{\Gamma}=T \ln \left(t-t^{\prime}\right)$ it is clear that $y$ can be larger than $O(1)$ only if $\hat{\Gamma}>\Gamma_{0}$ in which case it is equal to the separation, $y$, of the two minima with probability $2 p(1-p)$ and small otherwise. Thus to obtain the distribution $\tilde{Q}\left(y, t, t^{\prime}\right)$ for $y$ in the scaling regime, one must sum over all barriers smaller than $\hat{\Gamma}=T \ln \left(t-t^{\prime}\right)$ (the larger ones contribute only to the already dominant delta function part of $Q\left(y, t, t^{\prime}\right)$ ). Thus, using (170), we find in the scaling regime of fixed $\alpha=\frac{\ln \left(t-t^{\prime}\right)}{\ln t^{\prime}}<1$ and $y /\left(T \ln t^{\prime}\right)^{2}$ :

$$
\begin{aligned}
\tilde{Q}\left(y, t, t^{\prime}\right) & =C(T) \int_{0}^{\hat{\Gamma}} d \Gamma_{0} K_{\Gamma^{\prime}}\left(y, \Gamma_{0}\right) \\
& =2\left[1+\frac{y}{\left(T \ln t^{\prime}\right)^{2}}\right] \frac{T}{\left(T \ln \left(t-t^{\prime}\right)\right)^{3}} G\left[\frac{y}{\left(T \ln \left(t-t^{\prime}\right)\right)^{2}}\right]
\end{aligned}
$$

where we have defined:

$$
G(X)=4 \pi^{2} \sum_{n=1}^{\infty} n^{2} e^{-X n^{2} \pi^{2}}=\frac{1}{\sqrt{\pi} X^{3 / 2}} \sum_{m=-\infty}^{+\infty}\left(1+\frac{2 m^{2}}{X}\right) e^{-\frac{m^{2}}{X}}
$$

Note that the factor $C(T)=2 \int_{0}^{+\infty} d w e^{-w / T} /\left(1+e^{-w / T}\right)^{2}=T$ arises from the fact that the distribution of $w$ is constant around $w=0$.

The above result is consistent with previous observations in the case of finite, but large, $t-t^{\prime}$ where moments $\int d y y^{k} \tilde{Q}\left(y, t, t^{\prime}\right)$ were argued [16] to grow as $(T \ln (t-$ $\left.\left.t^{\prime}\right)\right)^{2 k-1}$ for $k>1 / 2$ and be bounded for $k<1 / 2$. Here we obtain, in addition, the behavior for more separated times with positive $\left(\ln \left(t-t^{\prime}\right) / \ln t^{\prime}\right)<1$.

In the biased case we find similarly (see Appendix E1)

$$
R_{\Gamma}^{ \pm}\left(\zeta, l, x, \Gamma_{0}\right)=\theta\left(\Gamma-\Gamma_{0}\right) \theta(l-x) P_{\Gamma}^{ \pm}(\zeta, l-x) \frac{1}{\Gamma_{0}^{4}} \hat{r}\left(x / \Gamma_{0}^{2}\right) e^{-x \delta^{2}}
$$

where $\hat{r}(X)$ is the same function (170) as in the symmetric case. From this we obtain, as above, the probability $K_{\Gamma^{\prime}}^{ \pm}\left(y, \Gamma_{0}\right)$ that the the starting point happens to belong to a renormalized valley at $\Gamma^{\prime}=T \ln t^{\prime}$ which possesses two degenerate minima separated by a distance $y$ and a barrier $\Gamma_{0}$. We find

$$
K_{\Gamma^{\prime}}^{ \pm}\left(y, \Gamma_{0}\right)=2\left(1+\frac{y}{\bar{l}_{\Gamma^{\prime}}}\right) \frac{1}{\Gamma_{0}^{4}} \hat{r}\left(\frac{y}{\Gamma_{0}^{2}}\right) e^{-y \delta^{2}}
$$

and thus, integrating over the barriers $\Gamma_{0}<\hat{\Gamma}$ yields the distribution of displacements $y=\left|x(t)-x\left(t^{\prime}\right)\right|$ in the presence of a small bias. We thereby obtain that in the scaling regime where the three scaling variables $y /\left(T \ln \left(t-t^{\prime}\right)\right)^{2}, \delta T \ln t^{\prime}$ and $\alpha=\ln \left(t-t^{\prime}\right) / \ln t^{\prime}$ are held fixed, the distribution of displacements is dominated by the rare events with valleys with two degenerate minima and with 


$$
\tilde{Q}^{ \pm}\left(y, t, t^{\prime}\right) \sim 2\left(1+\frac{\delta^{2} y}{\sinh ^{2}\left(\delta T \ln t^{\prime}\right)}\right) \frac{T}{\left(T \ln \left(t-t^{\prime}\right)\right)^{3}} G\left(\frac{y}{\left(T \ln \left(t-t^{\prime}\right)\right)^{2}}\right) e^{-y \delta^{2}}
$$

where, interestingly, $G$ is the same function (174) as in the symmetric case. This is because the rare events that dominate are those in which the relevant part of the landscape is almost symmetric.

\section{G. crossover at $t \sim t^{\prime}$}

So far we have studied separately the regime $t-t^{\prime} \sim t^{\prime \alpha}(\alpha>1)$ and the regime $t-t^{\prime} \sim t^{\prime \alpha}(\alpha<1)$. For completeness let us mention what happens (for the symmetric case) in the crossover regime $t-t^{\prime} \sim t^{\prime}$. In this regime, the distribution of displacements $y=\left|x(t)-x\left(t^{\prime}\right)\right|$ takes the form of a sum of two contributions.

First, it was found in (158) that in the limit $\alpha \rightarrow 1^{+}$this distribution is controlled by barriers of order $\Gamma^{\prime} \approx \Gamma$. For closer times, $\Gamma-\Gamma^{\prime} \sim O(T)$ one must consider more precisely the jumping process over the barrier. Asssociating a single relaxation time $\tau=e^{\left(\Gamma^{\prime}+\epsilon\right) / T}$ with the barrier of height $\Gamma^{\prime}+\epsilon$ one finds that the contribution of these events to the the distribution of $y$ takes the form, (in addition to a piece proportional to $\delta(y))$ :

$$
Q_{1}\left(y, t, t^{\prime}\right)=f\left(t, t^{\prime}\right) \frac{1}{\left(T \ln t^{\prime}\right)^{3}} \mathcal{H}\left(Y=\frac{y}{\left(T \ln t^{\prime}\right)^{2}}\right)
$$

where the Laplace transform of $\mathcal{H}(Y)$ was defined in (158). The coefficient $f\left(t, t^{\prime}\right)$ is obtained by noting that the probability that the particle jumps between $t^{\prime}$ and $t$ is $\left(e^{-t^{\prime} / \tau}-e^{-t / \tau}\right)$ and that the distribution of $\epsilon$ is uniform around $\epsilon=0$ with density $1 / \Gamma:$

$$
f\left(t, t^{\prime}\right)=\int_{-\infty}^{+\infty} d \epsilon\left[\exp \left(-e^{-\epsilon / T}\right)-\exp \left(-\frac{t}{t^{\prime}} e^{-\epsilon / T}\right)\right]=T \ln \frac{t}{t^{\prime}}
$$

The second contribution comes from the events discussed in the previous subsection, corresponding to degenerate wells (Fig. 6). These are dominant for $\alpha<1$ and they also give a contribution for $\alpha=1$, which must be added to (177). The corresponding contribution $Q_{2}\left(y, t, t^{\prime}\right)$ to the distribution of displacements is simply given by the limit $\alpha \rightarrow 1$ of equation (173) which corresponds to setting $\left.\ln \left(t-t^{\prime}\right) \approx \ln t^{\prime}\right)$.

Putting this all together we give the explicit expressions for the second moment of $y$ in the various regimes which can be obtained from $Q=Q_{1}+Q_{2}$ :

$$
\begin{aligned}
& \overline{\left\langle\left(x(t)-x\left(t^{\prime}\right)\right)^{2}\right\rangle} \approx \\
& T\left(T \ln \left(t-t^{\prime}\right)\right)^{3}\left[\frac{8}{45}+\frac{48}{945}\left(\frac{\ln \left(t-t^{\prime}\right)}{\ln t^{\prime}}\right)^{2}\right] \quad t-t^{\prime} \sim t^{\prime \alpha}, \alpha<1 \\
& T\left(T \ln t^{\prime}\right)^{3}\left(\frac{8}{35}+\frac{272}{315} \ln \left(\frac{t}{t^{\prime}}\right)\right) \quad t-t^{\prime} \sim t^{\prime} \\
& \left(T \ln t^{\prime}\right)^{4} F_{2}\left[\frac{\ln t}{\ln t^{\prime}}\right] \quad t-t^{\prime} \sim t^{\prime \alpha}, \alpha>1
\end{aligned}
$$

where the function $F_{2}[\alpha]$ is given in (153). 


\section{H. Rare events in the single time-diffusion front}

In this Section we examine further the rare events which produce subdominant corrections to the results from the RSRG. As discussed in Section IIB2, although subdominant, these corrections give the principal contribution to some observables when the leading contribution vanishes. This is the case for the thermal width of the diffusion front analyzed in IIIA, since this is zero in the effective dynamics. We now examine this in the symmetric model.
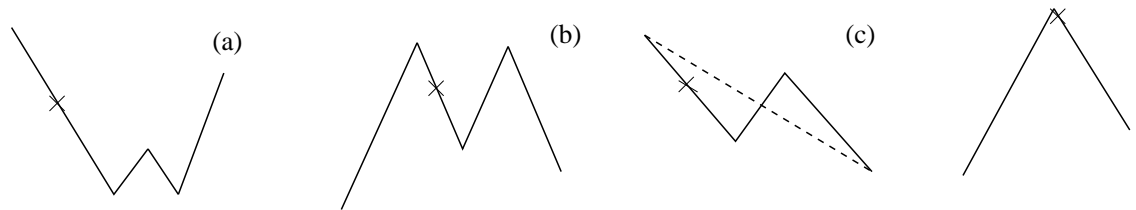

(d)

FIG. 7. Rare events which contribute to to the thermal width of the diffusion front. The starting point is indicated by a cross. (a) Valley with two degenerate minima. (b) Almost degenerate barriers. (c) Valley just being decimated with a barrier $\Gamma+\epsilon$. (d) Rare event of higher order with the starting point near the upper edge of a bond.

The possible rare events which contribute to the splitting of the thermal packet are indicated in Fig. 77. The most important ones, all occurring with probabilities of order $1 / \Gamma$, are the following. In case (a) the starting point belongs to a valley with two degenerate minima: this is the equilibrium situation already considered in the previous Section. In case (b) the splitting is due to the starting point being in a valley with two almost degenerate barriers: at scales when the packet overcomes the barriers, the packet will split between the two wells located on either sides, an intrinsically nonequilibrium phenomenon. Note that if the packet is split at $t$ (as in (b)) the probability that it remains split until a later time $\tilde{t}$ decays as $(\ln t / \ln \tilde{t})^{2}$. In case (c) the walker at $\Gamma$ belongs to a valley with a barrier $\Gamma+\epsilon$, with $\epsilon \sim O(T)$ positive or negative. In this case the thermal packet is already split at $\Gamma$ between two valleys. There are of course other rare events: for instance the one illustrated as case (d), when the starting point is near the upper edge of a bond; this also corresponds to an out of equilibrium situation, but it occurs with a smaller probability $O\left(1 / \Gamma^{2}\right)$.

Let us estimate in more detail the probabilities $Q_{\Gamma}^{(a)}(y), Q_{\Gamma}^{(b)}(y)$ and $Q_{\Gamma}^{(c)}(y)$ associated respectively with events (a), (b) and (c) in Fig. 7, that the packet be split at $\Gamma$ with a fixed separation $y$ between the two parts of the packet. Let us start with events (a) and (b) which can be treated similarly.

Events of type (a): We have already computed in (171) the probability $K_{\Gamma}\left(y, \Gamma_{0}\right)$ that the origin belongs at $\Gamma$ to a renormalized valley having two degenerate minima separated by a distance $y$ and a barrier $\Gamma_{0}$. Integrating over the barrier $\Gamma_{0}$ one gets:

$$
Q_{\Gamma}^{(a)}(y)=\int_{0}^{\Gamma} d \Gamma_{0} K_{\Gamma}\left(y, \Gamma_{0}\right)=2\left(1+\frac{y}{\Gamma^{2}}\right) \frac{1}{\Gamma^{3}} G\left(\frac{y}{\Gamma^{2}}\right)
$$

where the scaling function $G$ has been introduced in (174).

Events of type (b): Here we need to compute the probability $Q_{\Gamma}^{(b)}(y)$ that the origin belongs to a configuration of type $(b)$ with a distance $y$ between the two minima. We first compute the probability $R_{\Gamma}\left(l_{1}, y_{1}\right)$ that a bond at scale $\Gamma$ has a length $l_{1}$ and two degenerate minima separated by a distance $y_{1}$ (170) 


$$
R_{\Gamma}\left(l_{1}, y_{1}\right)=\int_{0}^{\infty} d \zeta \int_{0}^{\Gamma} d \Gamma_{0} R_{\Gamma}\left(\zeta, l_{1}, y_{1}, \Gamma_{0}\right)=P_{\Gamma}\left(l_{1}-y_{1}\right) \frac{1}{\Gamma^{3}} G\left(\frac{y_{1}}{\Gamma^{2}}\right)
$$

and thus:

$$
\begin{aligned}
Q_{\Gamma}^{(b)}(y) & =\frac{2}{\Gamma^{2}} \int_{0}^{\infty} d l_{1} \int_{0}^{\infty} d l_{2} \int_{0}^{l_{1}} d y_{1} y_{1} R_{\Gamma}\left(l_{1}, y_{1}\right) P_{\Gamma}\left(l_{2}\right) \delta\left(y-\left(l_{1}+l_{2}\right)\right) \\
& =\frac{2}{\Gamma^{3}} \int_{0}^{\frac{y y}{\Gamma^{2}}} d Y_{1} Y_{1} G\left(Y_{1}\right)(P(.) * P(.))_{\lambda=\frac{y}{\Gamma^{2}}-Y_{1}}
\end{aligned}
$$

in terms of the scaled $P$ from Eq. (14).

In either case (a) or (b) the packet is split between two wells and the thermal distribution can be written, in a scaling sense, as a sum of two delta function peaks, of the form $p \delta\left(x-x_{1}\right)+(1-p) \delta\left(x-x_{2}\right)$ centered at each minima $x_{1}$ and $x_{2}$ with $x_{1}<x_{2},\left|x_{2}-x_{1}\right|=y$. In case (a), as before $p_{a}=1 /\left(1+e^{-w / T}\right)$ where $w$ is the free energy difference between the two minima, while in case (b) a simple estimate of the relative escape rates in Fig. 7 also leads to $p_{b}=1 /\left(1+e^{-v / T}\right)$ where $v$ is now the (effective free-) energy difference between the maxima.

Thus we can estimate the dominant large time behavior of the moments of the thermal width coming from the contributions of (a) and (b) which simply add to give:

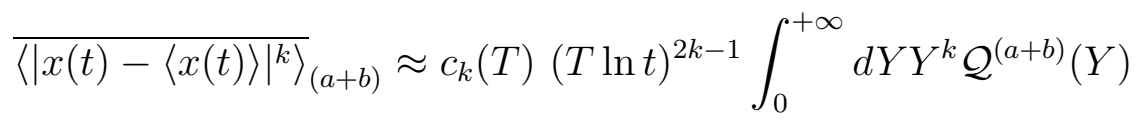

where the scaled distribution is

$$
\mathcal{Q}^{(a+b)}(Y)=2(1+Y) G(Y)+2 \int_{0}^{Y} d Y_{1} Y_{1} G\left(Y_{1}\right)(P(.) * P(.))_{\lambda=Y-Y_{1}}
$$

with $Y \equiv y / \Gamma^{2}$ The coefficients $c_{k}(T)$ can be computed using the fact the distributions of $w$ and of $v$ have constant density near 0 . This gives

$$
c_{k}(T)=\int_{-\infty}^{+\infty} d w\left(e^{-k w / T}+e^{-w / T}\right) /\left(1+e^{-w / T}\right)^{k+1}=\frac{2}{k} T .
$$

Using the Laplace transformed expression

$$
\begin{aligned}
& \int_{0}^{+\infty} d Y Y \mathcal{Q}^{(a+b)}(Y) e^{-s Y} \\
& =2 \partial_{s}\left(\sqrt{s} \operatorname{coth}(\sqrt{s})+\frac{1+\cosh ^{2}(\sqrt{s})}{\sinh (2 \sqrt{s})}\left(\frac{2}{\sinh (2 \sqrt{s})}-\frac{1}{\sqrt{s}}\right)\right)
\end{aligned}
$$

We must now consider the events (c) as shown in Fig. 7. The barrier that the particle must overcome to leave the valley is $\Gamma+\epsilon$. This corresponds to a single relaxation time $\tau=e^{(\Gamma+\epsilon) / T}$. Thus the probability that the particle is still in the valley at time $t=e^{\Gamma / T}$ is simply $p_{c}=\exp (-t / \tau)=\exp \left(-e^{-\epsilon / T}\right)$. The thermal distribution can then again be written, in a scaling sense, as $p_{c} \delta\left(x-x_{1}\right)+(1-$ $\left.p_{c}\right) \delta\left(x-x_{2}\right)$ where $x_{1}$ is the bottom of the valley being decimated and $x_{2}$ the bottom of the new valley. The distribution of $y=\left|x_{2}-x_{1}\right|$ is simply: 


$$
\begin{aligned}
Q_{\Gamma}^{(c)}(y) & =\frac{2}{\Gamma^{2}} \int_{0}^{\infty} d l_{1} \int_{0}^{\infty} d l_{2} \int_{0}^{\infty} d l_{3}\left(l_{1}+l_{2}\right) P_{\Gamma}\left(l_{1}\right) P_{\Gamma}\left(\zeta=0, l_{2}\right) P_{\Gamma}\left(l_{3}\right) \delta\left(y-\left(l_{2}+l_{3}\right)\right) \\
& =\frac{1}{\Gamma^{3}} \int_{0}^{\frac{y y}{\Gamma^{2}}} d \lambda_{2}\left(1+2 \lambda_{2}\right) P\left(\eta=0, \lambda_{2}\right) P\left(\frac{y}{\Gamma^{2}}-\lambda_{2}\right)
\end{aligned}
$$

The contributions of (c) to the moments thus read:

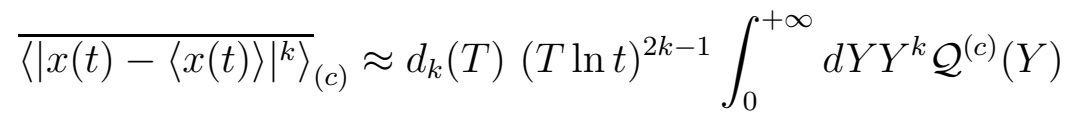

where the scaled distribution reads in Laplace transform:

$$
\int_{0}^{+\infty} d Y e^{-s Y} \mathcal{Q}^{(c)}(Y)=\frac{2}{\sinh (2 \sqrt{s})}\left(\operatorname{coth} \sqrt{s}+\sqrt{s}-\frac{1}{\sqrt{s}}\right)
$$

Using the fact that the distribution of $\epsilon$ is constant near zero, one obtains the coefficients $d_{k}(T)$ for $k \geq 1$ as:

$$
\begin{aligned}
& d_{k}(T)=\int_{-\infty}^{+\infty} d \epsilon\left[\exp \left(-k e^{-\epsilon / T}\right)\left(1-\exp \left(-e^{-\epsilon / T}\right)\right)+\exp \left(-e^{-\epsilon / T}\right)\left(1-\exp \left(-e^{-\epsilon / T}\right)\right)^{k}\right] \\
& =T\left[\ln \left(1+\frac{1}{k}\right)+\sum_{p=1}^{k}(-1)^{1+p} C_{k}^{p} \ln (1+p)\right]
\end{aligned}
$$

Note that the above argument can be made indentically in the region $\epsilon<0$ and thus we have integrated $\epsilon$ from $-\infty$ to $+\infty$.

Our final result for the moments are obtained as the sum of (186) and (190) and can be computed using the Laplace transforms in (188) and (191). Let us give the explicit resulting expression for the the lowest moments:

$$
\begin{aligned}
& \overline{\langle|x(t)-\langle x(t)\rangle|\rangle} \approx \frac{2}{45}(68+41 \ln 2) T(T \ln t) \\
& \overline{\left\langle x(t)^{2}\right\rangle-\langle x(t)\rangle^{2}} \approx \frac{4}{315}(95+68 \ln 2) T(T \ln t)^{3}
\end{aligned}
$$

It would be interesting to measure these quantities in numerical simulations and test these predictions.

Note that the above formula (186,190) give the leading behavior for moments with $k>1 / 2$ which grow with time, while the moments for $k<1 / 2$ are expected to be finite and non-universal as in [16]. This can be compared with the work of Golosov [26], who showed the existence of an infinite time limit distribution for $y(t)=x(t)-$ $\langle x(t)\rangle$ and gave an explicit formula in the case of a continuum Brownian potential $U(x)$ (which corresponds here to the limit $\sigma \rightarrow 0$ where additional universality holds). It is easy to see from [26], as well as from more general arguments, that this distribution has a tail $1 / y^{3 / 2}$ at large $y$. Indeed, from Eqs. (174, 183, 185) we see that for $t \rightarrow \infty$ so that $1 \ll y \ll \Gamma^{2}$,

$$
Q_{\Gamma}^{(a)+(b)+(c)}(y) \approx Q_{\Gamma}^{(a)}(y) \approx \frac{2}{\sqrt{\pi} y^{3 / 2}} .
$$


Thus moments $\left\langle y(t)^{k}\right\rangle$ for $k<1 / 2$ should be finite and determined from short scales, while moments for $k>1 / 2$ should diverge as $t \rightarrow \infty$. Our results include the large but finite time behavior and thus go beyond those results of 26.

To conclude, note that the rare events in (7) which contribute to the width of the thermal packet are also the one which play a dominant role in the aging dynamics in the regimes $\alpha \leq 1$. We have seen that $(a)$ and $(c)$ are the one which contribute to $Q\left(y, t, t^{\prime}\right)$ in these regimes. The event $(b)$ does not contribute to $Q\left(y, t, t^{\prime}\right)$ (since the particle cannot jump back to the degenerate valley) but would have to be considered to evaluate $P\left(x, t, x^{\prime}, t^{\prime}\right)$ in these regimes as well.

\section{FINITE SIZE PROPERTIES OF SINAI'S MODEL}

In this Section we apply the RSRG procedure to a finite size system with various boundary conditions and obtain exact results for the approach to equilibrium of several quantities.

\section{A. RG for a finite size system}

In order to follow the general measure for a finite size landscape one needs to introduce the set of functions representing probabilities $N_{\Gamma, L}^{b}\left(l_{1} ; \zeta_{2}, l_{2} ; \zeta_{3}, l_{3} ; \ldots ; \zeta_{b-1}, l_{b-1} ; l_{b}\right)$ in an ensemble of systems of length $L$ with $b$ the number of bonds and with barriers $\zeta_{i}=F_{i}-\Gamma$ and lengths $l_{i}$. Note that we will not keep track of $\zeta_{1}$ and $\zeta_{b}$ as these will effectively be $\pm \infty$ depending on the boundary conditions. The normalization condition reads:

$$
Z_{L}=\sum_{b} \int_{\zeta_{i}, l_{i}} N_{\Gamma, L}^{b}\left(l_{1} ; \zeta_{2}, l_{2} ; . . \zeta_{b-1}, l_{b-1} ; l_{b}\right)=1
$$

Note that under decimation one follows separately $b=2,4, \ldots$ or $b=1,3,5 \ldots$, depending on the type of boundary conditions studied here either reflecting $(\mathrm{R})$ or absorbing (A). Let us write the RG equation for a finite size system, choosing for definiteness the case denoted RR below of two reflecting boundaries - as explained in section [IB 3, in this case the first and last bonds have infinite barrier and cannot be decimated:

$$
\begin{aligned}
& \left(\partial_{\Gamma}-\sum_{k=2}^{b-1} \partial_{\zeta_{k}}\right) N_{\Gamma, L}^{b}\left(l_{1} ; \zeta_{2}, l_{2} ; \ldots \zeta_{b-1}, l_{b-1} ; l_{b}\right)= \\
& \int_{z, l+l^{\prime}+l^{\prime \prime}=l_{1}} N_{\Gamma, L}^{b+2}\left(l ; 0, l^{\prime} ; z, l^{\prime \prime} ; \zeta_{2}, l_{2} ; \ldots ; \zeta_{b-1}, l_{b-1} ; l_{b}\right)+ \\
& \sum_{k=2}^{b-1} \int_{z, l+l^{\prime}+l^{\prime \prime}=l_{k}} N_{\Gamma, L}^{b+2}\left(l_{1} ; \zeta_{2}, l_{2} ; \ldots ; \zeta_{k-1}, l_{k-1} ; z, l ; 0, l^{\prime} ; \zeta_{k}-z, l^{\prime \prime} ; \zeta_{k+1}, l_{k+1} ; \ldots ; \zeta_{b-1}, l_{b-1} ; l_{b}\right) \\
& +\int_{z, l+l^{\prime}+l^{\prime \prime}=l_{b}} N_{\Gamma, L}^{b+2}\left(l_{1} ; \zeta_{2}, l_{2} ; \ldots ; \zeta_{b-1}, l_{b-1} ; z, l ; 0, l^{\prime} ; l^{\prime \prime}\right) .
\end{aligned}
$$

There exists a quasi-decoupled solution - for Laplace transformed distributions - of this equation (197) (as was also found in the case of the RTFIC [22]), which reads: 


$$
\begin{aligned}
& N_{\Gamma, L}^{b}\left(l_{1} ; \zeta_{2}, l_{2} ; \ldots \zeta_{b-1}, l_{b-1} ; l_{b}\right)=E_{\Gamma}^{+}\left(l_{1}\right) P_{\Gamma}^{-}\left(\zeta_{2}, l_{2}\right) P_{\Gamma}^{+}\left(\zeta_{3}, l_{3}\right) \ldots \\
& \ldots P_{\Gamma}^{-}\left(\zeta_{b-2}, l_{b-2}\right) P_{\Gamma}^{+}\left(\zeta_{b-1}, l_{b-1}\right) E_{\Gamma}^{-}\left(l_{b}\right) \bar{l}_{\Gamma} \delta\left(L-\sum_{i=1}^{b} l_{i}\right) \quad \text { b even } \geq 2
\end{aligned}
$$

where we have allowed for a bias towards the right, and $b$ is restricted to be even since we are dealing with the RR case (see Figure 8). In this formula (198) $P_{\Gamma}^{ \pm}(\zeta, l)$ are the bulk distributions satisfying (6), $\bar{l}_{\Gamma}$ the average length satisfying (8), and the $E_{\Gamma}^{ \pm}(l)$ satisfy the semi-infinite boundary RG (10). The integral of the measure $Z_{L}$ over all variables in (196) satisfies:

$$
\int_{0}^{+\infty} d L e^{-p L} Z_{L}=\bar{l}_{\Gamma} \frac{E^{-}(p) E^{+}(p)}{1-P^{-}(p) P^{+}(p)}=\bar{l}_{\Gamma} \frac{u^{+}(0) u^{-}(0)}{u^{+}(p) u^{-}(p)-U^{+}(p) U^{-}(p)}=\frac{1}{p}
$$

where we have used (22, 35), and thus $Z_{L}=1$ so the finite size measure is correctly normalized.

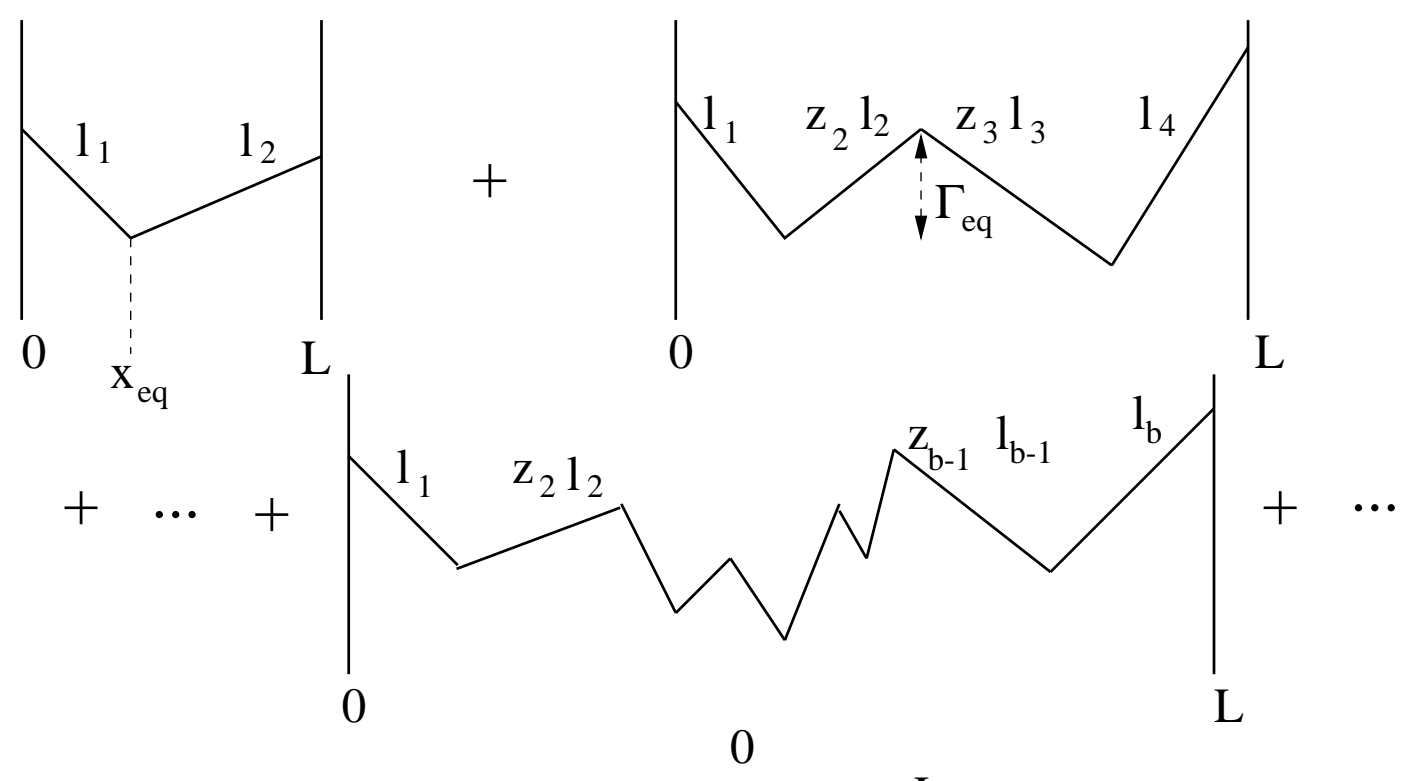

FIG. 8. Schematic of a finite size system of fixed length $L$ with reflecting boundaries (case RR).

In the case of two absorbing boundaries AA, the solution of the corresponding $\mathrm{RG}$ equations is obtained by simply exchanging + and - in (198) (b remains even). In the case $\mathrm{RA}$ the solution reads:

$$
\begin{aligned}
& N_{\Gamma, L}^{b}\left(l_{1} ; \zeta_{2}, l_{2} ; \ldots \zeta_{b-1}, l_{b-1} ; l_{b}\right)=E_{\Gamma}^{+}\left(l_{1}\right) P_{\Gamma}^{-}\left(\zeta_{2}, l_{2}\right) P_{\Gamma}^{+}\left(\zeta_{3}, l_{3}\right) \\
& \ldots P_{\Gamma}^{+}\left(\zeta_{b-2}, l_{b-2}\right) P_{\Gamma}^{-}\left(\zeta_{b-1}, l_{b-1}\right) E_{\Gamma}^{+}\left(l_{b}\right) \bar{l}_{\Gamma} \delta\left(L-\sum_{i=1}^{b} l_{i}\right) \quad b \text { odd } \geq 3
\end{aligned}
$$

together with the term $b=1$, which corresponds to the final state with a single $(+)$ bond over the whole system (all particles having been absorbed by the right boundary), and has for probability: 


$$
N_{\Gamma, L}^{1}=\int_{0}^{\Gamma} d \Gamma^{\prime} \int_{l_{1}, l_{2}, l_{3}} N_{\Gamma^{\prime}, L}^{3}\left(l_{1} ; \zeta_{2}=0, l_{2} ; l_{3}\right)
$$

Finally, the solution in the AR case can be obtained by exchanging + and - in (200).

\section{B. Evolution towards equilibrium in a system with reflecting boundaries}

Let us start by studying the equilibrium and the approach to equilibrium in the system of size $L$ with two reflecting boundaries. The equilibrium state corresponds to the large $\Gamma$ limit of the measure $(196)$. In that limit only the term $b=2$ (198) survives and it corresponds to equilibrium in a single renormalized valley. It will be reached, as illustrated in Fig. 8, when the last bulk bond is decimated at some $\Gamma=\Gamma_{e q}=T \ln t_{e q}$, with a certain sample to sample distribution for the equilibrium time $t_{e q}$, which we now compute.

\section{Distribution of equilibration time}

The probability for a sample to reach equilibrium between $\Gamma_{e q}$ and $\Gamma_{e q}+d \Gamma_{e q}$, i.e., the probability that the slowest relaxation time $t_{e q}$ be such that $\Gamma_{e q}<T \ln t_{e q}<$ $\Gamma_{e q}+d \Gamma_{e q}$, is

$$
\rho_{L}\left(\Gamma_{e q}\right)=\partial_{\Gamma_{e q}} \int_{l_{1}, l_{2}} N_{\Gamma_{e q}, L}^{2}\left(l_{1}, l_{2}\right)=\partial_{\Gamma_{e q}}\left(\bar{l}_{\Gamma_{e q}} E_{\Gamma_{e q}}^{+}(.) *_{L} E_{\Gamma_{e q}}^{-}(.)\right)
$$

and using (35), the Laplace transform with respect to the system size $L$ is

$$
\int_{0}^{\infty} d L e^{-p L} \rho_{L}\left(\Gamma_{e q}\right)=\partial_{\Gamma_{e q}}\left(\frac{1}{\left(p+\delta^{2}\right) \operatorname{coth}^{2}\left[\Gamma_{e q} \sqrt{p+\delta^{2}}\right]-\delta^{2}}\right) .
$$

For zero bias, one introduces the scaling variable

$$
w=\frac{T \ln t_{e q}}{\sqrt{L}}
$$

and finds that it is distributed as:

$$
\begin{aligned}
P(w) & =\frac{2}{w^{3}} \sum_{n=-\infty}^{+\infty}\left(2 \pi^{2}\left(n+\frac{1}{2}\right)^{2} \frac{1}{w^{2}}-1\right) e^{-\frac{\pi^{2}}{w^{2}}\left(n+\frac{1}{2}\right)^{2}} \\
& =\frac{2}{\sqrt{\pi} w^{2}} \sum_{m=-\infty}^{+\infty}(-1)^{m}\left(1-2 m^{2} w^{2}\right) e^{-m^{2} w^{2}}
\end{aligned}
$$

In the presence of a bias one can compute, e.g., the average:

$$
\overline{T \ln t_{e q}}=\overline{\Gamma_{e q}}=2 \sqrt{\frac{L}{\pi}} \int_{0}^{1} d u e^{-\delta^{2} L u^{2}} \ln \left(\frac{1}{u}\right)
$$




\section{Distribution of equilibrium position $x_{e q}$}

The probability that the bottom of the single remaining equilibrated valley is at $x=x_{e q}$ can be obtained as

$$
Q_{L}\left(x_{e q}\right)=N_{\Gamma \rightarrow \infty, L}^{2}\left(x_{e q}, l_{2}\right)=\left(\bar{l}_{\Gamma} E_{\Gamma}^{+}\left(x_{e q}\right) E_{\Gamma}^{-}\left(L-x_{e q}\right)\right)_{\Gamma \rightarrow \infty},
$$

which leads simply to:

$$
Q_{L}\left(x_{e q}\right)=e^{+}\left(x_{e q}\right) e^{-}\left(L-x_{e q}\right)
$$

where

$$
e^{ \pm}(x)=L T_{p \rightarrow x}^{-1}\left(\frac{1}{u_{\Gamma=\infty}^{ \pm}(p)}\right)=L T_{p \rightarrow x}^{-1} \frac{1}{\sqrt{p+\delta^{2}} \mp \delta} .
$$

Thus we get $e^{+}(x)=e^{-}(x)+2 \delta$ with

$$
e^{-}(x)=\frac{1}{\sqrt{\pi x}} e^{-x \delta^{2}}-\frac{2 \delta}{\pi} \int_{0}^{\infty} d v \frac{e^{-x \delta^{2}\left(1+v^{2}\right)}}{1+v^{2}} .
$$

At small $x$ one has $e^{ \pm}(x) \sim \frac{1}{\sqrt{x}}$ while for large $x, e^{+}(x) \approx 2 \delta$ and $e^{-}(x) \sim e^{-x \delta^{2}}$. Thus in the biased case with $L \gg 1 / \delta^{2}$ in equilibrium the particle is confined within a distance $y=L-x_{e q} \sim 1 / \delta^{2}$ near the left boundary distributed as $2 \delta e^{-}(y)$. In the symmetric case $\delta=0$ the equilibrium position is distributed over the whole system as:

$$
Q_{L}\left(x_{e q}\right)=\frac{1}{\pi \sqrt{x_{e q}\left(L-x_{e q}\right)}}
$$

which has a simple probabilistic interpretation in terms of the landscape random walk confined to $U(x)>U_{\min }=U\left(x_{e q}\right)$ on both sides of $x_{e q}$.

Finally, we obtain the joint distribution of equilibrium position $x_{e q}$ and equilibrium time $\Gamma_{e q}=T \ln t_{e q}$ :

$$
P_{L}\left(\Gamma_{e q}, x_{e q}\right)=\partial_{\Gamma_{e q}}\left(\bar{l}_{\Gamma_{e q}} E^{+}\left(x_{e q}\right) E^{-}\left(L-x_{e q}\right)\right)
$$

where $E^{ \pm}$was computed in (35, 37).

\section{First passage times}

\section{With a reflecting boundary}

Let us compute the probability $S_{x_{0}, L}(\Gamma)$ that a walker starting at $x_{0}$ is still alive at $\Gamma$ in the presence of an absorbing boundary at $x=0$ and a reflecting boundary at $x=L$. It can be expressed as an average over the measure AR (196, 200) $S_{x_{0}, L}(\Gamma)=\left\langle\theta\left(x_{0}-l_{1}\right)\right\rangle$. Thus its Laplace transform with respect to $L$ reads

$$
\int_{0}^{\infty} d L e^{-p L} S_{x_{0}, L}(\Gamma)=\frac{P_{\Gamma}(p)}{p E_{\Gamma}(p)} \int_{0}^{x_{0}} d l_{1} e^{-p l_{1}} E_{\Gamma}\left(l_{1}\right) .
$$


In the particular case where the starting point coincides with the reflecting boundary $\left(x_{0}=L\right)$ it is simpler to obtain the first passage time $T_{L L}$ at $x=0$. In that case, the probability to be absorbed coincides with the probability $\rho_{L}(\Gamma)$ that the last decimation occurs in the AR system at $\Gamma$; from (202):

$$
\rho_{L}(\Gamma)=\partial_{\Gamma} N_{\Gamma, L}^{1}=\bar{l}_{\Gamma} E_{\Gamma}^{+}(.) *_{L} P_{\Gamma}^{-}(0, .) *_{L} E_{\Gamma}^{+}(.) .
$$

In the symmetric case we thus obtain that the scaled first passage time variable $w=T \frac{\ln T_{L L}}{\sqrt{L}}$ is distributed as:

$s(w)=\frac{2 \pi}{w^{3}} \sum_{n=-\infty}^{\infty}(-1)^{n}\left(n+\frac{1}{2}\right) e^{-\frac{\pi^{2}}{w^{2}}\left(n+\frac{1}{2}\right)^{2}}=\frac{2}{\sqrt{\pi}} \sum_{m=-\infty}^{\infty}(-1)^{m}\left(m+\frac{1}{2}\right) e^{-w^{2}\left(m+\frac{1}{2}\right)^{2}}$.

We note that the distribution of $T_{L L}$ was obtained previously by a completely different method in 46 . Here we also recover $\ln \left(\overline{\left(T_{L L}\right)^{q}}\right) \sim q^{2} L$, i.e., that the first passage time is a strongly fluctuating quantity [47].

\section{With an absorbing boundary}

Let us now consider an absorbing boundary at $x=L$. We first compute the probability $p_{0}\left(x_{0}, L\right)$ that the walker starting at $x_{0}$ reaches $x=0$ before $x=L$. Since the final state of the AA system consists of two absorbing zones associated with each boundaries, the first one $\left[0, x_{e q}\right]$ and the second $\left[x_{e q}, L\right]$ where $x_{e q}$ is distributed as in (212). Thus, in presence of a bias applied in the direction \pm the result reads:

$$
p_{0}^{ \pm}\left(x_{0}, L\right)=\int_{x_{0}}^{L} d x e^{\mp}(x) e^{ \pm}(L-x)
$$

where $e \mp(x)$ was computed in (211). In the symmetric case this gives $p_{0}\left(x_{0}, L\right)=$ $\frac{1}{\pi} \operatorname{Arccos}\left(2 \frac{x_{0}}{L}-1\right)$.

One can also compute the survival probability $S_{x_{0}, L}(\Gamma)$ of a walker starting at $x_{0}$ in the presence of two absorbing boundaries at $x=0$ and $x=L$. It is obtained as an average over the measure (E5) of the finite size system AA as

$$
S_{x_{0}, L}(\Gamma)=\overline{\left\langle\theta\left(x_{0}-l_{1}\right) \theta\left(L-l_{1}-x_{0}\right)\right\rangle}
$$

and thus reads:

$S_{x_{0}, L}(\Gamma)=L T_{p \rightarrow L / \Gamma^{2}, q \rightarrow x_{0} / \Gamma^{2}}^{-1} \frac{\tanh (\sqrt{p+q}) \tanh (\sqrt{p})}{q \sqrt{p} \sqrt{p+q}}\left(\frac{1}{\sinh ^{2}(\sqrt{p})}-\frac{1}{\sinh ^{2}(\sqrt{p+q})}\right)$.

Note that the distribution of times $t_{\text {last }}$ at which all particles have left a given AA sample is identical to the one computed in (206) as $\ln t_{\text {last }}=\ln t_{e q}$. 


\section{Averaged diffusion front}

We first discuss a system bounded by two reflecting walls at $x=0$ and $x=L$. The full averaged diffusion front $\overline{\operatorname{Prob}_{0 L}\left(x, t \mid x_{0}, 0\right)}$ for a walker starting at $x_{0}$ at $t=0$ is computed in Appendix $\mathrm{G}$ for both biased and symmetric cases.

In the symmetric case it takes a scaling form $\overline{\operatorname{Prob}_{0 L}\left(x, t \mid x_{0}, 0\right)}=\frac{1}{\Gamma^{2}} q_{\lambda}\left(X \mid X_{0}\right)$ as a function of the rescaled variables $X=x / \Gamma^{2}, X_{0}=x_{0} / \Gamma^{2}$ and the rescaled length of the system $\lambda=L / \Gamma^{2}$ where $\Gamma=T \ln t$. The Laplace transform $\tilde{q}\left(p, p_{0}, q\right)=$ $\int_{0}^{+\infty} d \lambda \int_{0}^{\lambda} d X \int_{0}^{\lambda} d X_{0} e^{-\left(p X+p_{0} X_{0}+q \lambda\right)} q_{\lambda}\left(X \mid X_{0}\right)$ of the rescaled front is:

$$
\tilde{q}\left(p, p_{0}, q\right)=\frac{\tilde{P}_{p+p_{0}+q} \tilde{P}_{p+q}-\tilde{P}_{p_{0}+q} \tilde{P}_{q}}{p_{0} q\left(p+p_{0}+q\right) \tilde{E}_{p+p_{0}+q} \tilde{E}_{q}}+\frac{1}{p_{0} q} \frac{\tilde{E}_{q+p}}{\tilde{E}_{q}}-\frac{1}{p_{0}\left(p+p_{0}+q\right)} \frac{\tilde{E}_{p_{0}+q}}{\tilde{E}_{p+p_{0}+q}}
$$

where $\tilde{P}_{p}=1 / \cosh (\sqrt{p})$ and $\tilde{E}_{p}=\tanh (\sqrt{p}) / \sqrt{p}$. In the limit $L \rightarrow+\infty$ we can obtain the averaged front in a semi-infinite space with a reflecting wall at $x=0$ as:

$$
\lim _{q \rightarrow 0}\left(q \tilde{q}\left(p, p_{0}, q\right)\right)=\frac{\tilde{P}_{p+p_{0}} \tilde{P}_{p}-\tilde{P}_{p_{0}}}{p_{0}\left(p+p_{0}\right) \tilde{E}_{p+p_{0}}}+\frac{1}{p_{0}} \tilde{E}_{p}
$$

The corresponding formula for AA and RA are given in Appendix G.

There is a case where the diffusion front in a finite sample takes a particularly simple form. This is when the starting point coincides with the reflecting boundary $x_{0}=0$. The calculation of the Appendix simplifies as one then has that $x=l_{1}$ where $l_{1}$ is the length of the first bond. In the RR (or RA) case:

$$
\overline{\operatorname{Prob}_{0 L}\left(x, t \mid x_{0}=0,0\right)}=E_{\Gamma}^{+}(x) \phi_{\Gamma}^{R, A}(L-x)
$$

with

$$
\begin{aligned}
\phi_{\Gamma}^{R}(x) & =L T_{p \rightarrow x}^{-1} \frac{1}{p E_{\Gamma}^{+}(p)} \\
\phi_{\Gamma}^{A}(x) & =L T_{p \rightarrow x}^{-1} \frac{P_{\Gamma}^{-}(p)}{p E_{\Gamma}^{-}(p)}
\end{aligned}
$$

In the symmetric case one finds simply:

$$
\begin{aligned}
& \phi_{\Gamma}^{R}(x)=L T_{p \rightarrow x}^{-1} \frac{\Gamma \operatorname{coth}(\Gamma \sqrt{p})}{\sqrt{p}}=2 \sum_{n=0}^{\infty} e^{-n^{2} \pi^{2} \frac{x}{\Gamma^{2}}} \\
& \phi_{\Gamma}^{A}(x)=L T_{p \rightarrow x}^{-1} \frac{\Gamma}{\sqrt{p} \sinh (\Gamma \sqrt{p})}=2 \sum_{n=0}^{\infty}(-1)^{n} e^{-n^{2} \pi^{2} \frac{x}{\Gamma^{2}}} .
\end{aligned}
$$

\section{RESULTS FOR FOKKER PLANCK AND ASSOCIATED SCHRÖDINGER OPERATOR}

It is also interesting to obtain results, via the RSRG, for the random Schrödinger operator associated with the Sinai diffusion problem. We first recall the connection between these two problems. In this Section we set $T=1$. 


\section{A. From Fokker Planck to Schrödinger operator}

In a given environment $U(x)$ the probability distribution for the position of a particle $P\left(x, t \mid x_{0}, 0\right)$ satisfies the Fokker-Planck equation (in the continuum):

$$
\left.\partial_{t} P\left(x, t \mid x_{0}, 0\right)=\partial_{x}\left(\partial_{x}+U^{\prime}(x)\right) P\left(x, t \mid x_{0}, 0\right)\right)=-H_{F P} P\left(x, t \mid x_{0}, 0\right)
$$

with the initial condition $P\left(x, t \rightarrow 0 \mid x_{0}, 0\right) \rightarrow \delta\left(x-x_{0}\right)$. As is well known, setting

$$
\mathcal{G}\left(x, t \mid x_{0}, 0\right)=e^{\left(U(x)-U\left(x_{0}\right)\right) / 2} P\left(x, t \mid x_{0}, 0\right)
$$

one obtains the following imaginary time Schrödinger equation for the Green function $\mathcal{G}\left(x, t \mid x_{0}, 0\right)$

$$
\partial_{t} \mathcal{G}\left(x, t \mid x_{0}, 0\right)=\left(\partial_{x}^{2}+\frac{1}{2} U^{\prime \prime}(x)-\frac{1}{4} U^{\prime}(x)^{2}\right) \mathcal{G}\left(x, t \mid x_{0}, 0\right)=-H_{S} \mathcal{G}\left(x, t \mid x_{0}, 0\right)
$$

with the initial condition $\mathcal{G}\left(x, t \mid x_{0}, 0\right) \rightarrow \delta\left(x-x_{0}\right)$. This is the standard form for the Schrödinger operator $H_{S}$ associated with a diffusion process. It can be factorized as $H_{S}=Q^{\dagger} Q$ with

$$
Q=\partial_{x}+U^{\prime}(x) / 2
$$

and

$$
Q^{\dagger}=-\partial_{x}+U^{\prime}(x) / 2
$$

and thus has a real positive spectrum $E_{n}$. The Fokker Planck operator $H_{F P}$ is nonhermitian but has the same real positive spectrum, with right and left eigenfunctions $\Phi_{n}^{R}(x)$ and $\Phi_{n}^{L}(x)$ associated with $E_{n}$. They are related to the eigenfunctions $\psi_{n}(x)$ of the Schrödinger operator by $\Phi_{n}^{R}(x)=e^{-U(x) / 2} \psi_{n}(x)$ and $\Phi_{n}^{L}(x)=e^{U(x) / 2} \psi_{n}(x)$.

In the next two sections we use some of the results obtained previously for the Sinai diffusion process to obtain results for the Schrödinger and Fokker Planck operators.

\section{B. Averaged Green's function for the Schrödinger operator}

Interestingly, one can obtain the averaged Green's function of the Schrödinger operator (227) from a slight variation of the previous calculation for the dynamics inside a well of Section VIF. The physical reason is that in Sinai's model the particle tends to jump to and occupy lower accessible wells, with weight $e^{-U(x)}$ near the bottom. As a result one can show that, due to the the exponential factor in (227), the dominant contribution in the average over disorder of (227) comes from rare configurations in which the point $x$ and the point 0 are at about the same potential. The calculation is sketched in Appendix $\mathbb{E}$. The result is

$$
\overline{\mathcal{G}(x, 0, t)}=\frac{2}{\Gamma^{5}} G\left(\frac{x}{\Gamma^{2}}\right)
$$

with $\Gamma=\ln t$ with the scaling function $G(X)$ given by (174). 
In the case of a small bias $\delta>0$ the result becomes:

$$
\overline{\mathcal{G}(x>0,0, t)}=\overline{\mathcal{G}(x<0,0, t)}=\frac{2}{\Gamma^{5}}\left(\frac{\Gamma \delta}{\sinh (\Gamma \delta)}\right)^{2} e^{-\delta^{2}|x|} G\left(|x| / \Gamma^{2}\right)
$$

which is valid in the usual scaling regime $\Gamma \delta$ and $x / \Gamma^{2}$ fixed with $\Gamma=\ln t$ large. Note that this averaged Green's function Eq. (230), is closely related to the average Green's functions of a one-dimensional lattice fermion problem with random nearest neighbor hopping, $t_{n}=\bar{t}+\delta t_{n}$ as computed recently by L. Balents and M.P.A. Fisher [32]. In particular, the inverse Laplace transform of Eq. (230), $\overline{\mathcal{G}\left(x, x_{0}, E\right)}$ which is a function of the wave functions of $\mathcal{H}_{s}$ at energy $E$, is equivalent to the Green's function of the Fermi problem at energy $\epsilon=\sqrt{E}$ with $x-x_{0} \equiv n-n_{0}$ even, (corresponding to $\psi_{R}+\psi_{L}$ in terms of the right and left moving fermions of ref. [32]). This is related, in the Sinai problem, to the dominance of the averaged Green's function by $x$ and $x_{0}$ both at bottoms of valleys which correspond to even sites. The random hopping, $(-1)^{n} \delta t_{n}$ corresponds to $U^{\prime}(x) / 2$ in the Sinai problem.

By methods similar to those used in the present paper, one can obtain much more information about the statistical properties of eigenfunctions and Green's functions of both problems. These will be analyzed in ref. 48.

\section{CONCLUSIONS}

In this paper we have developed a powerful real space renormalization group (RSRG) method procedure for models of diffusion in one-dimensional random potentials which belong to the universality class of the Sinai model. This method is simple to implement, physically transparent and allows one to obtain exact results for universal quantities.

The RSRG was first applied to recover, as a check of its validity, the single time diffusion front for the rescaled position $x(t) /(T \ln t)^{2}$ obtained previously by Kesten and Golosov [12,27]. In addition we obtained the diffusion front in presence of a small bias in the crossover region.

The study of persistence properties, i.e., probabilities of return to the origin and their associated decay exponents, showed that in disordered systems distinctions must be made between recurrence properties of thermally averaged trajectories $\langle x(t)\rangle$ (exponent $\bar{\theta}$ ) and single particle trajectories (exponent $\theta$ ). Nontrivial exponents (e.g. $\bar{\theta}=(3-\sqrt{5}) / 4$ ) were obtained for thermally averaged trajectories, a novel and unexpected feature of the Sinai model. The distribution of number of returns $k$ was found to be strongly peaked in the rescaled variable, $g$, at $g=k / \ln (T \ln t)=1 / 3$ but with multifractal tails characterized by an exponent $\theta(g)$. It was shown that single-run averages $\frac{1}{t} \int_{0}^{t} x\left(t^{\prime}\right) d t^{\prime}$ obey the same scaling for $g<1 / 3$, but with deviations on the larger than typical $(g>1 / 3)$ side of the distribution due to rare events which were analyzed. We found that at each return to the origin, the thermally averaged trajectory loses some memory of the past. This allowed us to compute exactly the probability distribution of the complete sequence of return times. By contrast the successive jumps of $\langle x(t)\rangle$ exhibit persistent correlations which we have studied in detail. Much of the analysis was extended to the case of a small applied bias. 
Single particle properties, such as return probabilities, distributions of first passage times and of maximum displacement, were obtained by studying the RSRG in the presence of boundaries. The first meeting time distribution of two independent particles was also obtained. Extensions to large but finite size systems was studied, for reflecting or absorbing boundary conditions. The distributions of equilibration time and position, of the first passage times in presence of boundaries and the finite size diffusion front were all obtained.

A second set of results concerned aging dynamics. The scaling form of the joint distribution of positions $x\left(t^{\prime}\right)$ and $x(t)$ at two times $t^{\prime}<t$ was obtained explicitly in Laplace transforms. This two-time diffusion front was found to possess an overall $\ln t / \ln t^{\prime}$ scaling, with an interesting singularity at $x(t)=x\left(t^{\prime}\right)$. Explicit expressions of several moments and correlation functions were obtained. In the presence of a bias, our single time diffusion results (the distribution of $x / t^{\mu}$ being related to a Levy distribution) and our two-time aging results (with a $t / t^{\prime}$ scaling) are consistent with known exact results and with the phenomenological description in terms of an effective directed model with an algebraic distribution of waiting times. But in addition we have obtained the full crossover between the symmetric and biased aging scaling forms. Our aging results are also consistent with the numerical simulations and qualitative arguments of [16].

We have also obtained several quantities which are controlled by rare events such as renormalized valleys with degenerate minima or degenerate barriers. These can be studied systematically as subdominant contributions in the RSRG. From them, we computed the fluctuations in the thermal width of the single time diffusion front (i.e., moments such as $\overline{\left\langle x^{2}(t)\right\rangle-\langle x(t)\rangle^{2}} \sim T(T \ln t)^{3}$ ), the two-time diffusion front in the quasi-equilibrium regime (for $t-t^{\prime} \sim t^{\prime \alpha}, \alpha<1$ ).

This work exhibits the relationships which exist between the Sinai model and problems such as quantum spin chains with disorder: both can be treated via very similar RSRG methods. Although observables of physical interest are often different in each of these models, some interesting connections, have appeared-e.g., between persistence properties of Sinai model and magnetization in the random transverse field Ising model. The RSRG methods enable one to consider this class of models in a unified way. Since the method allows one to check its own range of validity, it may shed light on different universality classes. The averaged imaginary time Green function of a related random Schrödinger problem was found as a side benefit.

In conclusion, the model studied here provides an all too rare explicit example of a zero temperature glassy fixed point where detailed non-equilibrium quantities can be obtained. Qualitatively similar behavior should be expected in systems where, as in Sinai's model, the dynamics consists of jumps over large barriers between partially equilibrated configurations. The detailed understanding of physics in the simple onedimensional case studied here perhaps encourages hope that new methods - exact or approximate-based on similar ideas can be developed for more complex glassy systems. As a start, we have already applied the methods introduced here to more complex one-dimensional models, in particular the non-equilibrium dynamics of the classical random field Ising model [30], as well as reaction diffusion models with disorder [31]. Furthermore, recent work on random quantum Ising models in two and three dimension 49. suggest that in at least some systems the type of behavior found here are not particular to one dimension. 


\section{ACKNOWLEDGMENTS}

One of us (DSF) acknowledges support from the National Science Foundation via DMR9630064, DMS9304580, and Harvard University's MRSEC.

\section{APPENDIX A: AUXILIARY VARIABLE RG RULE, SYMMETRIC CASE}

In this Appendix we study the general RG rule $m^{\prime}=a m_{1}+b m_{2}+c m_{3}$ upon decimation of link 2 (see Fig. 1). We introduce the rescaled variable $\mu=m / \Gamma^{\psi}$ where $\psi$ is an unkown exponent and look for the fixed point joint probability distribution $P(\eta, \mu)$ that is a solution of

$$
\begin{aligned}
& 0=\left((1+\eta) \partial_{\eta}+1+\psi\left(\mu \partial_{\mu}+1\right)\right) P(\eta, \mu) \\
& +\int_{0}^{\infty} d \mu_{1} \int_{0}^{\infty} d \mu_{2} \int_{0}^{\infty} d \mu_{3} P\left(0, \mu_{2}\right) P\left(., \mu_{1}\right) *_{\eta} P\left(., \mu_{3}\right) \delta\left(\mu-\left(a \mu_{1}+b \mu_{2}+c \mu_{3}\right)\right)
\end{aligned}
$$

We have of course $P(\eta)=\int_{0}^{\infty} d \mu P(\eta, \mu)=e^{-\eta}$. The equation for the first moment $C(\eta)=\frac{1}{P(\eta)} \int_{0}^{\infty} d \mu \mu P(\eta, \mu)$ reads

$$
0=(1+\eta) \partial_{\eta} C(\eta)-(\eta+\psi) C(\eta)+(a+c) \int_{0}^{\eta} d \eta_{1} C\left(\eta_{1}\right)+b C(0) \eta
$$

It is useful to differentiate this equation with respect to $\eta$ to obtain

$$
0=(1+\eta) \partial_{\eta}^{2} C(\eta)+(1-\eta-\psi) \partial_{\eta} C(\eta)+(a+c-1) C(\eta)+b C(0)
$$

and to keep the boundary condition $C^{\prime}(0)=C(0) \psi$ at $\eta=0$.

For $a+c-1 \neq 0$ (the case for all the physical quantities discussed in this paper), it is convenient to set $y=1+\eta$ and $T(y)=C(\eta)+\frac{b}{a+c-1} C(0)$ so that that $T(y)$ satisfies the confluent hypergeometric equation

$$
0=y \partial_{y}^{2} T+(B-y) \partial_{y} T-A T(y)
$$

where $B=2-\psi$ and $A=1-a-c$, together with the boundary condition $T^{\prime}(y=$ $1)=\psi \frac{A}{A-b} T(y=1)$. Since we are looking for a well-behaved (i.e., not exponentially growing) solution at $\eta=\infty$, we find that $T(y)$ has to be proportional to the confluent hypergeometric function $U(A, B, y)$. To satisfy the boundary condition at $y=1$, we obtain, using the functional relation $U^{\prime}(A, B, 1)=U(A, B, 1)-U(A, B+1,1)$, that the exponent $\psi$ has to satisfy the equation

$$
0=\left(1-\psi \frac{a+c-1}{a+b+c-1}\right) U(1-a-c, 2-\psi, 1)-U(1-a-c, 3-\psi, 1)
$$

Note that in the particular case where $A=1-a-c=-1$, the function $U(-1, B, y)$ reduces to the linear function $-B+y$, and the equation for $\psi$ is simply quadratic $\psi(\psi-1)=1+b$ yielding $\psi=(1+\sqrt{5+4 b}) / 2$ as in [20].

In the text we use the ratio $m / \bar{l}_{\Gamma}$ which decays as $m / \bar{l}_{\Gamma} \sim \Gamma^{-\Phi}$ with $\Phi=2-\psi$. Both exponents $\psi$ and $\Phi$ depend explicitly on the coefficients $a, b, c$, of the RG rule. 


\section{APPENDIX B: AUXILIARY VARIABLE RG RULE, BIASED CASE}

We consider the auxiliary variables $m^{ \pm}$that evolve with the RG rules $m^{+}=$ $a^{+} m_{1}^{+}+b^{+} m_{2}^{-}+c^{+} m_{3}^{+}$upon decimation of an ascending link 2 and $m^{-}=a^{-} m_{1}^{-}+$ $b^{-} m_{2}^{+}+c^{-} m_{3}^{-}$upon decimation of a descending link 2 . We introduce the joint probability distributions $P_{\Gamma}^{ \pm}(\zeta, m)$ that evolve as

$$
\begin{aligned}
\left(\partial_{\Gamma}-\partial_{\zeta}\right) P_{\Gamma}^{ \pm}(\zeta, m)= & P_{\Gamma}^{ \pm}(\zeta, m) \int_{0}^{\infty} d m_{2}\left(P_{\Gamma}^{ \pm}\left(0, m_{2}\right)-P_{\Gamma}^{\mp}\left(0, m_{2}\right)\right) \\
+ & \int_{0}^{\infty} d m_{1} \int_{0}^{\infty} d m_{2} \int_{0}^{\infty} d m_{3} P_{\Gamma}^{\mp}\left(0, m_{2}\right) \\
& P_{\Gamma}^{ \pm}\left(., m_{1}\right) *_{\zeta} P_{\Gamma}^{ \pm}\left(., m_{3}\right) \delta\left(m-\left(a^{ \pm} m_{1}+b^{ \pm} m_{2}+c^{ \pm} m_{3}\right)\right) .
\end{aligned}
$$

We have $P_{\Gamma}^{ \pm}(\zeta)=\int_{0}^{\infty} d m P_{\Gamma}^{ \pm}(\zeta, m)=u_{\Gamma}^{ \pm} e^{-z u_{\Gamma}^{ \pm}}$. The equation for the first moments $C_{\Gamma}^{ \pm}(\zeta)=\frac{1}{P_{\Gamma}^{ \pm}(\zeta)} \int_{0}^{\infty} d m m P_{\Gamma}^{ \pm}(\zeta, m)$ is

$$
\left(\partial_{\Gamma}-\partial_{\zeta}\right) C_{\Gamma}^{ \pm}(\zeta)=u_{\Gamma}^{+} u_{\Gamma}^{-}\left[z\left(b^{ \pm} C_{\Gamma}^{\mp}(0)-C_{\Gamma}^{ \pm}(\zeta)\right)+\left(a^{ \pm}+c^{ \pm}\right) \int_{0}^{z} d \zeta^{\prime} C_{\Gamma}^{ \pm}\left(\zeta^{\prime}\right)\right]
$$

with $u_{\Gamma}^{+} u_{\Gamma}^{-}=\frac{\delta^{2}}{\sinh ^{2}(\Gamma \delta)}=1 / \bar{l}_{\Gamma}$

We study the simpler particular case when $a^{+}+c^{+}=2, a^{-}+c^{-}=2, b^{+}=$ $b^{-}=b$. Then the solutions $C_{\Gamma}^{+}(\zeta)=C_{\Gamma}^{-}(\zeta)=C_{\Gamma}(\zeta)$ are simply linear in $\zeta$ : $C_{\Gamma}(\zeta)=A_{\Gamma}+\zeta B_{\Gamma}$ and the coefficients satisfy

$$
\begin{aligned}
& B_{\Gamma}=\partial_{\Gamma} A_{\Gamma} \\
& \partial_{\Gamma}^{2} A_{\Gamma}=(1+b) u_{\Gamma}^{+} u_{\Gamma}^{-} A_{\Gamma}=(1+b) \frac{\delta^{2}}{\sinh ^{2}(\Gamma \delta)} A_{\Gamma}
\end{aligned}
$$

For $\delta=0$ we have already seen in Appendix $\mathrm{A}$ that the auxiliary variable $m$ grows as $\Gamma^{\psi(b)}$ with $\psi(b)=(1+\sqrt{5+4 b}) / 2$. Indeed for $\delta=0, A_{\Gamma} \propto \Gamma^{\psi(b)}$ is a solution of (B4). For $\delta>0$ following [21] we thus look for a solution of the scaling form:

$$
A_{\Gamma}=\delta^{-\psi(b)} A^{(b)}(\gamma=\delta \Gamma)
$$

where $A^{(b)}(\gamma)$ satisfies the equation:

$$
\partial_{\gamma}^{2} A^{(b)}(\gamma)=\frac{1+b}{\sinh ^{2}(\gamma)} A^{(b)}(\gamma)
$$

with the boundary condition $A^{(b)}(\gamma) \propto \gamma^{\psi}(b)$ as $\gamma \rightarrow 0$. Introducing the new variable $y=\operatorname{coth} \gamma$, we obtain the differential equation for the Legendre functions:

$$
\left(y^{2}-1\right) \frac{d^{2} A^{(b)}}{d y^{2}}+2 y \frac{d A^{(b)}}{d y}-(1+b) A^{(b)}=0 .
$$

The solution for $A^{(b)}(\gamma)$ with the above boundary condition is: 


$$
\begin{aligned}
A^{(b)}(\gamma) & =K Q_{\psi(b)-1}(\operatorname{coth} \gamma) \\
& =K^{\prime} \tanh (\gamma)^{\psi(b)} F\left(\frac{\psi(b)+1}{2}, \frac{\psi(b)}{2}, \psi(b)+\frac{1}{2}, \tanh (\gamma)^{2}\right)
\end{aligned}
$$

with $K^{\prime}=K \sqrt{\pi} 2^{-\psi(b)} \frac{\Gamma(\psi(b))}{\Gamma(\psi(b)+1 / 2)}$ where $K$ is a non-universal constant. The asymptotic behaviors are $A^{(b)}(\gamma) \simeq K^{\prime} \gamma^{\psi(b)}$ at small $\gamma$, and $A^{(b)}(\gamma)=$ $2 K^{\prime} \frac{\Gamma[\psi(b)+1 / 2]}{\Gamma[(\psi(b)+1) / 2] \Gamma[\psi(b) / 2]} \gamma$ at large $\gamma$. We can now compute the mean values of the variables $m^{ \pm}$

$$
\left\langle m^{ \pm}\right\rangle=\int_{0}^{\infty} d \zeta \int_{0}^{\infty} d m m P_{\Gamma}^{ \pm}(\zeta, m) \int_{0}^{\infty} d \zeta P_{\Gamma}^{ \pm}(\zeta) C_{\Gamma}^{ \pm}(\zeta)=A_{\Gamma}+\frac{\partial_{\Gamma} A_{\Gamma}}{u_{\Gamma}^{ \pm}}
$$

yielding (92) in the text.

\section{APPENDIX C: CORRELATION OF TIMES AND DIRECTIONS OF SUCCESSIVE JUMPS}

\section{Conditional probabilities of times of jumps forward and backward}

In this Appendix we compute the conditional probabilities $\rho_{\Gamma \Gamma^{\prime}}^{(f)}$ to make a jump forward at $\Gamma$ (respectively a jump backward) given that the last jump occurred at $\Gamma^{\prime}$. We define $D_{\Gamma, \Gamma^{\prime}}(F)$ as the probability to be on a descending bond of barrier $F$ given that the last jump of the effective dynamics occurred at $\Gamma^{\prime}$ (this jump was necessarily in the $(+)$ direction since the walker is on a descending bond). The initial condition is thus given by:

$$
\begin{aligned}
& D_{\Gamma^{\prime}, \Gamma^{\prime}}(F)=K \int_{\Gamma}^{\infty} d F_{1} \int_{0}^{\infty} d l_{1} \int_{0}^{\infty} d l_{2} \int_{\Gamma}^{\infty} d F_{3} \int_{0}^{\infty} d l_{3}\left(l_{1}+l_{2}\right) \\
& P_{\Gamma}\left(F_{1}, l_{1}\right) P_{\Gamma}\left(\Gamma, l_{2}\right) P_{\Gamma}\left(F_{3}, l_{3}\right) \delta\left(F-\left(F_{1}+F_{3}-\Gamma\right)\right)
\end{aligned}
$$

Indeed, the bond must be a new bond made, at $\Gamma^{\prime}$, out of three bonds, and the origin of the random walk must have been on either the first or the second bond in order to satisfy the condition that the last jump occurred at scale $\Gamma^{\prime}$. The normalization constant $K$ has to be choosen to ensure that $\int_{\Gamma}^{\infty} D_{\Gamma^{\prime}, \Gamma^{\prime}}(F)=1$. Introducing the rescaled variables $\eta=\frac{F-\Gamma}{\Gamma}$ and $\alpha=\frac{\Gamma}{\Gamma^{\prime}}$, we obtain that $D_{\alpha}(\eta)$ evolves according to

$$
\left(\alpha \partial_{\alpha}-(1+\eta) \partial_{\eta}-1\right) D_{\alpha}(\eta)=-2 D_{\alpha}(\eta)+\int_{0}^{\eta} d \eta^{\prime} D_{\alpha}\left(\eta^{\prime}\right) e^{-\left(\eta-\eta^{\prime}\right)}
$$

with the initial condition at $\alpha=1$ given from (C1) by $D_{\alpha=1}(\eta)=\left(\frac{\eta}{2}+\frac{\eta^{2}}{4}\right) e^{-\eta}$. The solution reads

$$
\begin{aligned}
D_{\alpha}(\eta) & =A_{\alpha} e^{-\eta}+\left(B_{\alpha}+C_{\alpha} \eta\right) e^{-\alpha \eta} \\
A_{\alpha} & =\frac{1}{2 \alpha^{2}}\left[5+\left(\frac{\left.\alpha^{2}+2 \alpha-2\right)}{(\alpha-1)^{2}}\right) e^{-(\alpha-1)}\right] \\
B_{\alpha} & =-\frac{1}{2}\left(\frac{\alpha}{\alpha-1}\right)^{2} e^{-(\alpha-1)} \\
C_{\alpha} & =-\frac{1}{2}\left(\frac{\alpha}{\alpha-1}\right) e^{-(\alpha-1)}
\end{aligned}
$$


The probability to make a forward jump at $\Gamma$ (i.e., in the $(+)$ direction) given that the last jump occured at $\Gamma^{\prime}$ (and by convention was in the $(+)$ direction) is

$$
\rho_{\Gamma, \Gamma^{\prime}}^{(f)}=P_{\Gamma}(\Gamma) \int_{\Gamma}^{\infty} d F D_{\Gamma, \Gamma^{\prime}}(F)=\frac{1}{\Gamma} \int_{0}^{\infty} d \eta D_{\alpha}(\eta)
$$

since the probability that the neighboring bond is decimated at $\Gamma$ is $P_{\Gamma}(\Gamma)=1 / \Gamma$. On the other hand the probability to make a backward jump at $\Gamma$ (i.e., in the (-) direction) given that the last jump occurred at $\Gamma^{\prime}$ is

$$
\rho_{\Gamma, \Gamma^{\prime}}^{(b)}=D_{\Gamma, \Gamma^{\prime}}(\Gamma)=\frac{1}{\Gamma} D_{\alpha}(0)
$$

which is the probability to decimate the bond we are interested in. The total probability to jump at $\Gamma$ in any direction given that the last jump occurred at $\Gamma^{\prime}$ must satisfy $\rho_{\Gamma, \Gamma^{\prime}}=\rho_{\Gamma, \Gamma^{\prime}}^{(f)}+\rho_{\Gamma, \Gamma^{\prime}}^{(b)}=-\partial_{\Gamma} \int_{\Gamma}^{\infty} d F D_{\Gamma, \Gamma^{\prime}}(F)$. These expressions, after substituting the above solution ([C6) yield the formulae (88) given in the text.

\section{Correlations in the sequence of times of successive forward and backward jumps}

A full calculation of all terms is quite involved and goes beyond the present paper. Here we indicate only the result for the two first elementary building blocks for the many jump correlations. The first one is

$$
\rho_{0}^{b b}\left(\Gamma_{1} \mid \Gamma_{0}\right) d \Gamma_{1}=\rho_{0}^{b b}\left(\alpha_{1}\right) d \alpha_{1}=\frac{d \alpha_{1}}{\alpha_{1}^{3}}\left(2-\left(1+\alpha_{1}\right) e^{-\left(\alpha_{1}-1\right)}\right)
$$

which is a scaling function of $\alpha_{1}=\Gamma_{1} / \Gamma_{0}$. Intermediate calculations also yield the probability that the second jump occurs at $\Gamma_{1}$ and is a forward jump given that the first one occurs at $\Gamma_{0}$ and is backward.

$$
\rho_{0}^{f b}\left(\Gamma_{1} \mid \Gamma_{0}\right) d \Gamma_{1}=\rho_{0}^{f b}\left(\alpha_{1}\right) d \alpha_{1}=\frac{d \alpha_{1}}{\alpha_{1}^{3}}\left(2-e^{-\left(\alpha_{1}-1\right)}\right) .
$$

The second elementary building block is given by:

$$
\begin{aligned}
& \rho_{1}^{b f b}\left(\Gamma_{2}, \Gamma_{1} \mid \Gamma_{0}\right) d \Gamma_{2} d \Gamma_{1}=\rho_{1}^{b f b}\left(\alpha_{1}, \alpha_{2}\right) d \alpha_{1} d \alpha_{2}=\frac{d \alpha_{1} d \alpha_{2}\left(2-e^{-\left(\alpha_{1}-1\right)}\right)}{\alpha_{1}^{3} \alpha_{2}^{3}\left(2-e^{-\left(\alpha_{1}-1\right)}\right)} \\
& \left(4-e^{-\left(\alpha_{1}-1\right)}-2\left(\alpha_{2}+1\right) e^{-\left(\alpha_{2}-1\right)}-\left(\alpha_{2}+1\right) \frac{e^{-\left(\alpha_{2}+\alpha_{1}-2\right)}}{\alpha_{1}-1}+\left(\alpha_{2} \alpha_{1}+1\right) \frac{e^{-\left(\alpha_{2} \alpha_{1}-1\right)}}{\alpha_{1}-1}\right)
\end{aligned}
$$

which is a scaling function of $\alpha_{1}=\Gamma_{1} / \Gamma_{0}$ and $\alpha_{2}=\Gamma_{2} / \Gamma_{1}$.

\section{APPENDIX D: DISTRIBUTION OF SEQUENCES OF RETURNS TO THE ORIGIN: BIASED CASE}

To compute the conditional probabilities $\rho^{ \pm}\left(\Gamma, \Gamma^{\prime}\right)$ of returns to the origin defined in the text (Section $\overline{I V F}$ ) we consider the probability $D_{\Gamma, \Gamma^{\prime}}^{ \pm}(\zeta)$ that a bond has barrier 
$z$ at $\Gamma$ and has not changed orientation since the scale $\Gamma^{\prime}$ where it was created. Its $\mathrm{RG}$ equation is

$$
\left(\partial_{\Gamma}-\partial_{\zeta}\right) D_{\Gamma, \Gamma^{\prime}}^{ \pm}(\zeta)=2 P_{\Gamma}^{\mp}(0) D_{\Gamma, \Gamma^{\prime}}^{ \pm}(.) *_{\zeta} P_{\Gamma}^{ \pm}(.)-2 P_{\Gamma}^{\mp}(0) D_{\Gamma, \Gamma^{\prime}}^{ \pm}(\zeta)
$$

with the initial condition $D_{\Gamma^{\prime}, \Gamma^{\prime}}^{ \pm}(\zeta)=\left(u_{\Gamma^{\prime}}^{ \pm}\right)^{2} \zeta e^{-\zeta u_{\Gamma^{\prime}}^{ \pm}}$. Since $P_{\Gamma}^{ \pm}(\zeta)=u_{\Gamma}^{ \pm} e^{-\zeta u_{\Gamma}^{ \pm}}$, the solution has the following form

$$
D_{\Gamma^{\prime}, \Gamma^{\prime}}^{ \pm}(\zeta)=\left(A_{\Gamma, \Gamma^{\prime}}+\zeta B_{\Gamma, \Gamma^{\prime}}\right) \frac{u_{\Gamma}^{\mp}}{u_{\Gamma^{\prime}}^{\mp}}\left(u_{\Gamma}^{ \pm}\right)^{2} e^{-\zeta u_{\Gamma}^{ \pm}}
$$

where the coefficients are

$$
\begin{aligned}
& A_{\Gamma, \Gamma^{\prime}}=\delta^{-1}\left(Q_{\phi-1}(\operatorname{coth} \gamma) P_{\phi-1}\left(\operatorname{coth} \gamma^{\prime}\right)-Q_{\phi-1}\left(\operatorname{coth} \gamma^{\prime}\right) P_{\phi-1}(\operatorname{coth} \gamma)\right) \\
& B_{\Gamma, \Gamma^{\prime}}=\frac{1}{\sinh ^{2} \gamma}\left(P_{\phi-1}^{\prime}(\operatorname{coth} \gamma) Q_{\phi-1}\left(\operatorname{coth} \gamma^{\prime}\right)-P_{\phi-1}\left(\operatorname{coth} \gamma^{\prime}\right) Q_{\phi-1}^{\prime}(\operatorname{coth} \gamma)\right)
\end{aligned}
$$

where $\gamma=\delta \Gamma, \gamma^{\prime}=\delta \Gamma^{\prime}, \phi=(1+\sqrt{5}) / 2$ and $Q_{\nu}(y)$ and $P_{\nu}(y)$ are associated Legendre functions: they are two linearly independent solutions of the equation (B7) (with $(1+b) \rightarrow \nu(1+\nu))$.

The probability for a $( \pm)$ link to be decimated at $\Gamma$ given that its last decimation occurred at $\Gamma^{\prime}$ is therefore

$$
\rho^{ \pm}\left(\Gamma, \Gamma^{\prime}\right)=-\partial_{\Gamma} \int_{0}^{\infty} d \zeta D_{\Gamma, \Gamma^{\prime}}^{ \pm}(\zeta)=\frac{u_{\Gamma}^{+} u_{\Gamma}^{-}}{u_{\Gamma^{\prime}}^{\mp}} u_{\Gamma}^{ \pm} A_{\Gamma, \Gamma^{\prime}}
$$

This leads to the equation (101) given in the text in terms of the the reduced variables $y=\operatorname{coth} \gamma$ and $y^{\prime}=\operatorname{coth} \gamma^{\prime}$.

\section{APPENDIX E: DYNAMICS WITHIN A WELL}

\section{Probability that a bond has degenerate minima}

Let us introduce the probability $S_{\Gamma}(\zeta, l, x, w)$ that: a given point (denoted $x_{0}$ in Fig. 9) belongs at $\Gamma$ to a bond of barrier $F=\Gamma+\zeta$, of length $l$, is at a distance $x$ from the min of the bond and is at a potential $w$ above the potential of the minimum of the bond. One has that by definition $0<x<l$ and its normalization with respect to $x$ and $w$ is $\int_{0}^{l} d x \int_{0}^{\Gamma+\zeta} d w S_{\Gamma}(\zeta, l, x, w)=l P_{\Gamma}(\zeta, l) / \int_{l} P_{\Gamma}(l)$, which is the probability that a given point belongs to a bond with $F, l$. The RG equation for $S_{\Gamma}(\zeta, l, x, w)$ reads:

$$
\begin{aligned}
& \left(\partial_{\Gamma}-\partial_{\zeta}\right) S_{\Gamma}(\zeta, l, x, w)=-2 P_{\Gamma}(0) S_{\Gamma}(\zeta, l, x, w)+P_{\Gamma}(0, .) *_{l} P_{\Gamma}(., .) *_{\zeta, l} S_{\Gamma}(., ., x, w) \\
& +\int_{\zeta_{1}, l_{1}, x_{1}, w_{1}, l_{2}, \zeta_{3}, l_{3}} S_{\Gamma}\left(\zeta_{1}, l_{1}, x_{1}, w_{1}\right) P_{\Gamma}\left(0, l_{2}\right) P_{\Gamma}\left(\zeta_{3}, l_{3}\right) \\
& \delta\left(\zeta-\left(\zeta_{1}+\zeta_{3}\right)\right) \delta\left(l-\left(l_{1}+l_{2}+l_{3}\right)\right) \delta\left(x-\left(x_{1}+l_{2}+l_{3}\right)\right) \delta\left(w-\left(w_{1}+\zeta_{3}\right)\right) \\
& +\int_{\zeta_{1}, l_{1}, x_{2}, w_{2}, l_{2}, \zeta_{3}, l_{3}} P_{\Gamma}\left(\zeta_{1}, l_{1}\right) S_{\Gamma}\left(0, l_{2}, x_{2}, w_{2}\right) P_{\Gamma}\left(\zeta_{3}, l_{3}\right) \\
& \delta\left(\zeta-\left(\zeta_{1}+\zeta_{3}\right)\right) \delta\left(l-\left(l_{1}+l_{2}+l_{3}\right)\right) \delta\left(x-\left(l_{1}+l_{2}-x_{2}\right)\right) \delta\left(w-\left(w_{2}+\zeta_{1}\right)\right)
\end{aligned}
$$


(a)

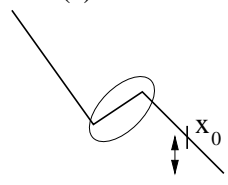

(b)

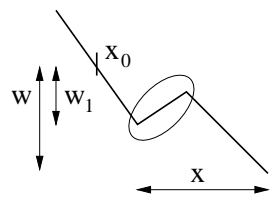

(c)

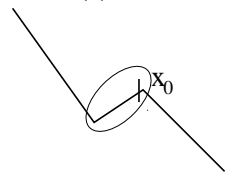

FIG. 9. Different terms which contribute to the RG equation for $S$.

where each term is described in Fig. (9) We notice that the evolution equation for $S_{\Gamma}(\zeta, l, x, w=0)$ decouples and leads to the form:

$$
S_{\Gamma}(\zeta, l, x, w=0)=\frac{2}{\Gamma^{2}}\left(\delta(x) P_{\Gamma}(\zeta, l)+R_{\Gamma}(\zeta, l, x)\right)
$$

where the delta-function part represents the probability that the point $x_{0}$ happens to be exactly at the bottom of the renormalized bond in which case $w=0$ by definition. The function $R_{\Gamma}(\zeta, l, x)$ is the probability that a renormalized bond at scale $\Gamma$ has $(\zeta, l)$ and a distinct degenerate minimum at a finite distance $x$. For small $x(x=O(1))$ this function is non-universal. We compute this function in the scaling regime $x \sim \Gamma^{2}$ where it is universal and of order $1 / \Gamma$. We use the rescaled variables $\eta=\zeta / \Gamma, \lambda=l / \Gamma^{2}$ and $X=x / \Gamma^{2}$ such that $R_{\Gamma}(\zeta, l, x)=\Gamma^{-6} R(\eta, \lambda, X)$ and obtain the following fixed point RG equation for $R$ :

$$
\begin{aligned}
& 0=\Gamma \partial_{\Gamma} R_{\Gamma}(\eta, \lambda, X)=\left((1+\eta) \partial_{\eta}+2 \lambda \partial_{\lambda}+2 X \partial_{X}+6\right) R(\eta, \lambda, X) \\
& +P(0, .) *_{\lambda} P(., .) *_{\eta, \lambda} R(., ., X)+2 P(\eta, \lambda-X)\left(P(0, .) *_{\lambda=X} P(0, .)\right)
\end{aligned}
$$

This equation was obtained by substituting the decomposition (E2) into (E1) in the spirit of an expansion in powers of $1 / \Gamma$, where the zeroth-order equation is satisfied by the delta-function part. The order $O(1 / \Gamma)$ equation yields the equation for $R_{\Gamma}(\zeta, l, x)$ where the delta-function part acts now as a source in the last two terms of (E1) leading to the last term in (E3). This term describes the probability that between $\Gamma$ and $\Gamma+d \Gamma$ a new bond with one degenerate minimum (a distance of order $\Gamma^{2}$ away from the lowest edge) is created via the decimation of a bond whose neighbor also has $\zeta \sim 0$ - cases (b) and (c) in Fig. 9 with $w=w_{1}=0$ ) - the probability of this is of order $1 / \Gamma$.

Before proceeding further, we notice that it is easy to also keep track of the barrier $\Gamma_{0}$ between the two degenerate minima. We define $R_{\Gamma}\left(\zeta, l, x, \Gamma_{0}\right)$ as the probability that a renormalized bond at scale $\Gamma$ has $(\zeta, l)$ and a distinct degenerate minimum at a finite distance $x$ separated from the minimum by a barrier $\Gamma_{0}$. It takes the scaling form $R_{\Gamma}\left(\zeta, l, x, \Gamma_{0}\right)=\Gamma^{-7} R_{\Gamma}(\eta, \lambda, X, u)$ with $u=\Gamma_{0} / \Gamma$. The normalization is $R_{\Gamma}(\eta, \lambda, X)=\int_{0}^{1} d u R_{\Gamma}(\eta, \lambda, X, u)$. The scaling form satisfies the fixed point $\mathrm{RG}$ equation:

$0=\Gamma \partial_{\Gamma} R(\eta, \lambda, X, u)=\left((1+\eta) \partial_{\eta}+2 \lambda \partial_{\lambda}+2 X \partial_{X}+u \partial_{u}+7\right) R(\eta, \lambda, X, u)$

$+P(0,.) *_{\lambda} P(.,.) *_{\eta, \lambda} R(., ., X, u)+2 P(\eta, \lambda-X)\left(P(0,.) *_{\lambda=X} P(0,).\right) \delta(u-1)$

where the last term corresponds to barriers $\Gamma_{0}=\Gamma$ created upon decimation.

Remarkably, one can find the complete solution of this equation in a factorized form: 


$$
R(\eta, \lambda, X, u)=P(\eta, \lambda-X) r(X, u)
$$

where $r(X, u)$ satisfies

$$
0=\left(2 X \partial_{X}+u \partial_{u}+4\right) r(X, u)+2\left(P(0, .) *_{\lambda=X} P(0, .)\right) \delta(u-1)
$$

whose solution is:

$$
\begin{aligned}
& r(X, u)=\theta(1-u) u^{-4} \hat{r}\left(X / u^{2}\right) \\
& \hat{r}(X)=2\left(P(0, .) *_{\lambda=X} P(0, .)\right)
\end{aligned}
$$

which, using (11), yields formula (170) given in the text.

The biased case can be studied similarly. The corresponding quantities (as usual \pm designate descending and ascending bonds, respectively) also satisfy $S_{\Gamma}^{ \pm}(\zeta, l, x, w=0)=\frac{1}{\bar{l}_{\Gamma}}\left(\delta(x) P_{\Gamma}^{ \pm}+R_{\Gamma}^{ \pm}(\zeta, l, x)\right)$ and one finds that the RG equation for $R_{\Gamma}^{ \pm}\left(\zeta, l, x, \Gamma_{0}\right)$ is

$$
\begin{aligned}
& \left(\partial_{\Gamma}-\partial_{\zeta}\right) R_{\Gamma}^{ \pm}\left(\zeta, l, x, \Gamma_{0}\right)=\left(P_{\Gamma}^{ \pm}(0)-P_{\Gamma}^{\mp}(0)\right) R_{\Gamma}^{ \pm}\left(F, l, x, \Gamma_{0}\right) \\
& +P_{\Gamma}^{\mp}(0, .) *_{l} P_{\Gamma}^{ \pm}(., .) *_{\zeta, l} R_{\Gamma}^{ \pm}\left(., ., x, \Gamma_{0}\right)+2 P_{\Gamma}^{ \pm}(\zeta, l-x) P_{\Gamma}^{\mp}(0, .) *_{x} P_{\Gamma}^{ \pm}(0, .) \delta\left(\Gamma-\Gamma_{0}\right)
\end{aligned}
$$

The solution again factorizes into

$$
R_{\Gamma}^{ \pm}\left(\zeta, l, x, \Gamma_{0}\right)=P_{\Gamma}^{ \pm}(\zeta, l-x) r_{\Gamma}\left(x, \Gamma_{0}\right)
$$

where $r_{\Gamma}\left(x, \Gamma_{0}\right)=2 \theta\left(\Gamma-\Gamma_{0}\right) P_{\Gamma_{0}}^{+}(0,.) *_{x} P_{\Gamma_{0}}^{-}(0,$.$) does not depend on the direction$ of the bias. Its Laplace transform is simply

$$
\int_{0}^{\infty} d x e^{-p x} r_{\Gamma}\left(x, \Gamma_{0}\right)=\theta\left(\Gamma-\Gamma_{0}\right) 2 U_{\Gamma_{0}}^{+}(p) U_{\Gamma_{0}}^{-}(p)=2 \theta\left(\Gamma-\Gamma_{0}\right) \frac{p+\delta^{2}}{\sinh ^{2}\left(\Gamma_{0} \sqrt{p+\delta^{2}}\right)}
$$

so that finally

$$
r_{\Gamma}\left(x, \Gamma_{0}\right)=\theta\left(\Gamma-\Gamma_{0}\right) \frac{1}{\Gamma_{0}^{4}} \hat{r}\left(\frac{x}{\Gamma_{0}^{2}}\right) e^{-x \delta^{2}}
$$

where $\hat{r}$ is the function for the symmetric case introduced in (170).

\section{Relationship to the associated Schrödinger operator Green's function}

The disorder averaged Green function defined in Section VIIIB is exactly related to the probability $S_{\Gamma}(\zeta, l, x, w)$ introduced above. In the symmetric case, one can restrict to $x>0$, and one has:

$$
\overline{\mathcal{G}(x, 0, t)}=\frac{1}{2} \int_{0}^{\infty} d \zeta \int_{0}^{\zeta+\Gamma} d w e^{-w / 2} \int_{x}^{\infty} d l S_{\Gamma}(\zeta, l, x, w)
$$

The factor $\frac{1}{2}$ is simply the probability to be on a descending bond $(x>0)$. This can be expressed using the rescaled variables $\zeta=\Gamma \eta, w=\Gamma u, l=\Gamma^{2} \lambda, x=\Gamma^{2} X$

and simplified using that for large $\Gamma$, we may replace $e^{-\Gamma u / 2}$ by $\frac{2}{\Gamma} \delta(u)$. Using (E2) one obtains: 


$$
\overline{\mathcal{G}(x, 0, t)}=\frac{2}{\Gamma^{5}} \int_{0}^{\infty} d \eta \int_{X}^{\infty} d \lambda R(\eta, \lambda, X) .
$$

Using (E5) one finds the result of the text (229).

In the biased case we obtain an expression for the averaged Green function in terms of the functions $S_{\Gamma}^{ \pm}(\zeta, l, x, 0)$

$$
\begin{aligned}
& \overline{\mathcal{G}(x>0,0, t)}=2 \int_{0}^{\infty} d \zeta \int_{|x|}^{\infty} d l S_{\Gamma}^{+}(\zeta, l,|x|, 0) \\
& \overline{\mathcal{G}(x<0,0, t)}=2 \int_{0}^{\infty} d \zeta \int_{|x|}^{\infty} d l S_{\Gamma}^{-}(F, l,|x|, 0)
\end{aligned}
$$

Using $(\overline{\text { E10 }})$ we finally get $\overline{\mathcal{G}(x>0,0, t)}=\overline{\mathcal{G}(x<0,0, t)}=\mathcal{G}(|x|, t)$ with

$$
\mathcal{G}(|x|, t)=\frac{2}{\bar{l}_{\Gamma}} \int_{0}^{\Gamma} d \Gamma_{0} r_{\Gamma}\left(|x|, \Gamma_{0}\right)=\frac{2}{\bar{l}_{\Gamma}} \frac{1}{\Gamma^{3}} G\left(\frac{|x|}{\Gamma^{2}}\right) e^{-|x| \delta^{2}}
$$

where the function $G$ has been introduced in (174), leading to formula (230) in the text.

\section{APPENDIX F: SOLUTION OF THE TWO TIME RG EQUATIONS}

In this Appendix we solve explicitly the RG equations (147) for the quantities

$\Omega_{\Gamma, \Gamma^{\prime}}^{\epsilon \epsilon^{\prime}}\left(\zeta, x_{L}, x_{R} ; x_{L}^{\prime}, x_{R}^{\prime}\right), \epsilon= \pm 1 \epsilon^{\prime}= \pm 1$ from which we can obtain the two-time diffusion front $\overline{\operatorname{Prob}\left(x t, x^{\prime} t^{\prime} \mid 00\right)}$. We consider the general biased case and discuss the particular limit of the symmetric case.

We first introduce the Laplace transforms:

$$
\begin{aligned}
& \hat{\Omega}_{\Gamma, \Gamma^{\prime}}^{\epsilon \epsilon^{\prime}}\left(\zeta, \mu, \nu ; \mu^{\prime}, \nu^{\prime}\right) \\
& =\int_{x_{L}^{\prime}>0, x_{R}^{\prime}>0, x_{L}>0, x_{R}>0} e^{-\mu^{\prime} x_{R}^{\prime}} e^{-\nu^{\prime} x_{L}^{\prime}} e^{-\mu\left(x_{R}-x_{R}^{\prime}\right)} e^{-\nu\left(x_{L}-x_{L}^{\prime}\right)} \Omega_{\Gamma, \Gamma^{\prime}}^{\epsilon \epsilon^{\prime}}\left(\zeta, x_{L}, x_{R} ; x_{L}^{\prime}, x_{R}^{\prime}\right)
\end{aligned}
$$

Since we consider large $\Gamma^{\prime}$ we can use the fixed point solution (22):

$$
P_{\Gamma}^{ \pm}(\zeta, \mu)=\int_{0}^{\infty} d l e^{-\mu l} P_{\Gamma}^{ \pm}(\zeta, l)=U_{\Gamma}^{ \pm}(\mu) e^{-\zeta u_{\Gamma}^{ \pm}(\mu)}
$$

The RG equations (147) can be then written in Laplace variables as:

$$
\begin{aligned}
& \left(\partial_{\Gamma}-\partial_{\zeta}\right) \hat{\Omega}_{\Gamma, \Gamma^{\prime}}^{ \pm \epsilon^{\prime}}\left(\zeta, \mu, \nu ; \mu^{\prime}, \nu^{\prime}\right)=-2 U_{\Gamma}^{\mp}(0) \Omega_{\Gamma, \Gamma^{\prime}}^{ \pm \epsilon^{\prime}}\left(\zeta, \mu, \nu ; \mu^{\prime}, \nu^{\prime}\right) \\
& +U_{\Gamma}^{+}(\mu) U_{\Gamma}^{-}(\mu) \int_{0}^{\infty} d \zeta_{1} e^{-\left(\zeta-\zeta_{1}\right) u_{\Gamma}^{ \pm}(\mu)} \hat{\Omega}_{\Gamma, \Gamma^{\prime}}^{ \pm \epsilon^{\prime}}\left(\zeta_{1}, \mu, \nu ; \mu^{\prime}, \nu^{\prime}\right) \\
& +U_{\Gamma}^{+}(\nu) U_{\Gamma}^{-}(\nu) \int_{0}^{\infty} d \zeta_{1} e^{-\left(\zeta-\zeta_{1}\right) u_{\Gamma}^{ \pm}(\nu)} \hat{\Omega}_{\Gamma, \Gamma^{\prime}}^{ \pm \epsilon^{\prime}}\left(\zeta_{1}, \mu, \nu ; \mu^{\prime}, \nu^{\prime}\right) \\
& +\hat{\Omega}_{\Gamma, \Gamma^{\prime}}^{\mp \epsilon^{\prime}}\left(0, \mu, \nu ; \mu^{\prime}, \nu^{\prime}\right) U_{\Gamma}^{ \pm}(\mu) U_{\Gamma}^{ \pm}(\nu) \int_{0}^{\infty} d \zeta_{1} e^{-\left(\zeta-\zeta_{1}\right) u_{\Gamma}^{ \pm}(\mu)} e^{-\zeta_{1} u_{\Gamma}^{ \pm}(\nu)}
\end{aligned}
$$


together with the initial conditions at $\Gamma=\Gamma^{\prime}$ given in (148) which become:

$$
\begin{aligned}
\hat{\Omega}_{\Gamma^{\prime}, \Gamma^{\prime}}^{\epsilon \epsilon^{\prime}}\left(\zeta, \mu, \nu ; \mu^{\prime}, \nu^{\prime}\right) & =\delta_{\epsilon \epsilon^{\prime}} \int_{0}^{\infty} d x_{L}^{\prime} \int_{0}^{\infty} d x_{R}^{\prime} e^{-\mu^{\prime} x_{R}^{\prime}} e^{-\nu^{\prime} x_{L}^{\prime}} \omega_{\Gamma^{\prime}}^{\epsilon^{\prime}}\left(\zeta, x_{R}^{\prime}, x_{L}^{\prime}\right) \\
& =\delta_{\epsilon \epsilon^{\prime}} \frac{1}{\bar{l}_{\Gamma^{\prime}}} \frac{1}{\mu^{\prime}-\nu^{\prime}}\left(U_{\Gamma^{\prime}}^{\epsilon^{\prime}}\left(\nu^{\prime}\right) e^{-\zeta u_{\Gamma^{\prime}}^{\epsilon^{\prime}}\left(\nu^{\prime}\right)}-U_{\Gamma^{\prime}}^{\epsilon^{\prime}}\left(\mu^{\prime}\right) e^{-\zeta u_{\Gamma^{\prime}}^{\epsilon^{\prime}}\left(\mu^{\prime}\right)}\right) .
\end{aligned}
$$

We look for solutions of the form

$$
\begin{aligned}
\hat{\Omega}_{\Gamma, \Gamma^{\prime}}^{\epsilon \epsilon^{\prime}}\left(\zeta, \mu, \nu ; \mu^{\prime}, \nu^{\prime}\right)= & A_{\Gamma, \Gamma^{\prime}}^{\epsilon \epsilon^{\prime}}\left(\mu, \nu ; \mu^{\prime}, \nu^{\prime}\right) e^{-\zeta u_{\Gamma}^{\epsilon}(\mu)}+B_{\Gamma, \Gamma^{\prime}}^{\epsilon \epsilon^{\prime}}\left(\mu, \nu ; \mu^{\prime}, \nu^{\prime}\right) e^{-\zeta u_{\Gamma}^{\epsilon}(\nu)} \\
& +C_{\Gamma, \Gamma^{\prime}}^{\epsilon \epsilon^{\prime}}\left(\mu, \nu ; \mu^{\prime}, \nu^{\prime}\right) e^{-\zeta u_{\Gamma^{\prime}}^{\epsilon^{\prime}}\left(\mu^{\prime}\right)}+D_{\Gamma, \Gamma^{\prime}}^{\epsilon \epsilon^{\prime}}\left(\mu, \nu ; \mu^{\prime}, \nu^{\prime}\right) e^{-\zeta u_{\Gamma^{\prime}}^{\epsilon^{\prime}}\left(\nu^{\prime}\right)} .
\end{aligned}
$$

It is useful to introduce the functions

$$
\begin{aligned}
& \theta_{\Gamma, \Gamma^{\prime}}^{\epsilon \epsilon^{\prime}}\left(\mu, \nu ; \mu^{\prime}, \nu^{\prime}\right)=\Omega_{\Gamma, \Gamma^{\prime}}^{\epsilon \epsilon \epsilon^{\prime}}\left(\zeta=0, \mu, \nu ; \mu^{\prime}, \nu^{\prime}\right) \\
& =A_{\Gamma, \Gamma^{\prime}}^{\epsilon \epsilon^{\prime}}\left(\mu, \nu ; \mu^{\prime}, \nu^{\prime}\right)+B_{\Gamma, \Gamma^{\prime}}^{\epsilon \epsilon^{\prime}}\left(\mu, \nu ; \mu^{\prime}, \nu^{\prime}\right)+C_{\Gamma, \Gamma^{\prime}}^{\epsilon \epsilon^{\prime}}\left(\mu, \nu ; \mu^{\prime}, \nu^{\prime}\right)+D_{\Gamma, \Gamma^{\prime}}^{\epsilon \epsilon^{\prime}}\left(\mu, \nu ; \mu^{\prime}, \nu^{\prime}\right) \\
& \sigma_{\Gamma, \Gamma^{\prime}}^{\epsilon \epsilon^{\prime}}\left(\mu, \nu ; \mu^{\prime}, \nu^{\prime}\right)=\int_{0}^{\infty} d \zeta \Omega_{\Gamma, \Gamma^{\prime}}^{\epsilon \epsilon^{\prime}}\left(\zeta, \mu, \nu ; \mu^{\prime}, \nu^{\prime}\right) \\
& =\frac{A_{\Gamma, \Gamma^{\prime}}^{\epsilon \epsilon}\left(\mu, \nu ; \mu^{\prime}, \nu^{\prime}\right)}{u_{\Gamma}^{\epsilon}(\mu)}+\frac{B_{\Gamma, \Gamma^{\prime}}^{\epsilon \epsilon^{\prime}}\left(\mu, \nu ; \mu^{\prime}, \nu^{\prime}\right)}{u_{\Gamma}^{\epsilon}(\nu)}+\frac{C_{\Gamma, \Gamma^{\prime}}^{\epsilon \epsilon^{\prime}}\left(\mu, \nu ; \mu^{\prime}, \nu^{\prime}\right)}{u_{\Gamma^{\prime}}^{\epsilon^{\prime}}\left(\mu^{\prime}\right)}+\frac{D_{\Gamma, \Gamma^{\prime}}^{\epsilon \epsilon^{\prime}}\left(\mu, \nu ; \mu^{\prime}, \nu^{\prime}\right)}{u_{\Gamma^{\prime}}^{\epsilon^{\prime}}\left(\nu^{\prime}\right)}
\end{aligned}
$$

For each initial condition indexed by one of $\epsilon^{\prime}= \pm 1$ it is most convenient to work with the eight functions $\theta^{\epsilon \epsilon^{\prime}}, \sigma^{\epsilon \epsilon^{\prime}}, C^{\epsilon \epsilon^{\prime}}$ and $D^{\epsilon \epsilon^{\prime}}$ with $\epsilon= \pm 1$.

We first consider the equations for $C^{\epsilon \epsilon^{\prime}}$ and $D^{\epsilon \epsilon^{\prime}}$. These equations are homogenous and thus easier to solve. The equations for $C^{\epsilon \epsilon^{\prime}}$ read

$$
\begin{aligned}
\partial_{\Gamma} C_{\Gamma, \Gamma^{\prime}}^{\epsilon \epsilon^{\prime}}\left(\mu, \nu ; \mu^{\prime}, \nu^{\prime}\right)= & \left(-2 U_{\Gamma}^{-}(0) \delta_{\epsilon,+1}-2 U_{\Gamma}^{+}(0) \delta_{\epsilon,-1}-u_{\Gamma^{\prime}}^{\epsilon^{\prime}}\left(\mu^{\prime}\right)+\frac{U_{\Gamma}^{-}(\mu) U_{\Gamma}^{+}(\mu)}{u_{\Gamma}^{\epsilon}(\mu)-u_{\Gamma^{\prime}}^{\epsilon^{\prime}}\left(\mu^{\prime}\right)}\right. \\
& \left.+\frac{U_{\Gamma}^{-}(\nu) U_{\Gamma}^{+}(\nu)}{u_{\Gamma}^{\epsilon}(\nu)-u_{\Gamma^{\prime}}^{\epsilon^{\prime}}\left(\mu^{\prime}\right)}\right) C_{\Gamma, \Gamma^{\prime}}^{\epsilon \epsilon^{\prime}}\left(\mu, \nu ; \mu^{\prime}, \nu^{\prime}\right)
\end{aligned}
$$

with initial conditions at $\Gamma=\Gamma^{\prime}$ indexed by $\epsilon^{\prime}$ :

$$
C_{\Gamma^{\prime}, \Gamma^{\prime}}^{\epsilon \epsilon^{\prime}}\left(\mu, \nu ; \mu^{\prime}, \nu^{\prime}\right)=-\delta_{\epsilon \epsilon^{\prime}} \frac{1}{\bar{l}_{\Gamma^{\prime}}} \frac{U_{\Gamma^{\prime}}^{\epsilon^{\prime}}\left(\mu^{\prime}\right)}{\mu^{\prime}-\nu^{\prime}} .
$$

Similarly the equations for $D^{ \pm}$read

$$
\begin{aligned}
\partial_{\Gamma} D_{\Gamma, \Gamma^{\prime}}^{\epsilon \epsilon^{\prime}}\left(\mu, \nu ; \mu^{\prime}, \nu^{\prime}\right)= & \left(-2 U_{\Gamma}^{-}(0) \delta_{\epsilon,+1}-2 U_{\Gamma}^{+}(0) \delta_{\epsilon,-1}-u_{\Gamma^{\prime}}^{\epsilon^{\prime}}\left(\nu^{\prime}\right)+\frac{U_{\Gamma}^{-}(\mu) U_{\Gamma}^{+}(\mu)}{u_{\Gamma}^{\epsilon}(\mu)-u_{\Gamma^{\prime}}^{\epsilon^{\prime}}\left(\nu^{\prime}\right)}\right. \\
& \left.+\frac{U_{\Gamma}^{-}(\nu) U_{\Gamma}^{+}(\nu)}{u_{\Gamma}^{\epsilon}(\nu)-u_{\Gamma^{\prime}}^{\epsilon^{\prime}}\left(\nu^{\prime}\right)}\right) D_{\Gamma, \Gamma^{\prime}}^{\epsilon \epsilon^{\prime}}\left(\mu, \nu ; \mu^{\prime}, \nu^{\prime}\right)
\end{aligned}
$$

with initial conditions at $\Gamma=\Gamma^{\prime}$ are indexed by $\epsilon^{\prime}$ :

$$
D_{\Gamma^{\prime}, \Gamma^{\prime}}^{\epsilon \epsilon^{\prime}}\left(\mu, \nu ; \mu^{\prime}, \nu^{\prime}\right)=\delta_{\epsilon \epsilon^{\prime}} \frac{1}{\bar{l}_{\Gamma^{\prime}}} \frac{U_{\Gamma^{\prime}}^{\epsilon^{\prime}}\left(\nu^{\prime}\right)}{\mu^{\prime}-\nu^{\prime}} .
$$


To find the solution one notices that each matrix element $C^{\epsilon \epsilon^{\prime}}$ and $D^{\epsilon \epsilon^{\prime}}$ satisfies its own differential equation. Thus, since the initial condition is diagonal in $\epsilon \epsilon^{\prime}$, the solution is also diagonal. It is found to be:

$$
\begin{aligned}
& C_{\Gamma, \Gamma^{\prime}}^{\epsilon \epsilon^{\prime}}\left(\mu, \nu ; \mu^{\prime}, \nu^{\prime}\right)= \\
& \frac{-\delta_{\epsilon \epsilon^{\prime}}}{\bar{l}_{\Gamma^{\prime}}} \frac{U_{\Gamma}^{\epsilon^{\prime}}(0)^{2}}{U_{\Gamma^{\prime}}^{\epsilon^{\prime}}(0)^{2}} \frac{U_{\Gamma^{\prime}}^{\epsilon^{\prime}}\left(\mu^{\prime}\right)}{\left(\mu^{\prime}-\nu^{\prime}\right)} \frac{\left(u_{\Gamma^{\prime}}^{\epsilon^{\prime}}\left(\mu^{\prime}\right)-u_{\Gamma^{\prime}}^{\epsilon^{\prime}}(\mu)\right)\left(u_{\Gamma^{\prime}}^{\epsilon^{\prime}}\left(\mu^{\prime}\right)-u_{\Gamma^{\prime}}^{\epsilon^{\prime}}(\nu)\right)}{\left(u_{\Gamma^{\prime}}^{\epsilon^{\prime}}\left(\mu^{\prime}\right)-u_{\Gamma}^{\epsilon^{\prime}}(\mu)\right)\left(u_{\Gamma^{\prime}}^{\epsilon^{\prime}}\left(\mu^{\prime}\right)-u_{\Gamma}^{\epsilon^{\prime}}(\nu)\right)} e^{-\left(\Gamma-\Gamma^{\prime}\right) u_{\Gamma^{\prime}}^{\epsilon^{\prime}}\left(\mu^{\prime}\right)} \\
& D_{\Gamma, \Gamma^{\prime}}^{\epsilon \epsilon^{\prime}}\left(\mu, \nu ; \mu^{\prime}, \nu^{\prime}\right)= \\
& \frac{\delta_{\epsilon \epsilon^{\prime}}}{\bar{l}_{\Gamma^{\prime}}} \frac{U_{\Gamma}^{\epsilon^{\prime}}(0)^{2}}{U_{\Gamma^{\prime}}^{\epsilon^{\prime}}(0)^{2}} \frac{U_{\Gamma^{\prime}}^{\epsilon^{\prime}}\left(\nu^{\prime}\right)}{\left(\mu^{\prime}-\nu^{\prime}\right)} \frac{\left(u_{\Gamma^{\prime}}^{\epsilon^{\prime}}\left(\nu^{\prime}\right)-u_{\Gamma^{\prime}}^{\epsilon^{\prime}}(\mu)\right)\left(u_{\Gamma^{\prime}}^{\epsilon^{\prime}}\left(\nu^{\prime}\right)-u_{\Gamma^{\prime}}^{\epsilon^{\prime}}(\nu)\right)}{\left(u_{\Gamma^{\prime}}^{\epsilon^{\prime}}\left(\nu^{\prime}\right)-u_{\Gamma}^{\epsilon^{\prime}}(\mu)\right)\left(u_{\Gamma^{\prime}}^{\epsilon^{\prime}}\left(\nu^{\prime}\right)-u_{\Gamma}^{\epsilon^{\prime}}(\nu)\right)} e^{-\left(\Gamma-\Gamma^{\prime}\right) u_{\Gamma^{\prime}}^{\epsilon^{\prime}\left(\nu^{\prime}\right)}}
\end{aligned}
$$

For each $\epsilon^{\prime}$ the four remaining functions $\theta^{ \pm, \epsilon^{\prime}}$ and $\sigma^{ \pm, \epsilon^{\prime}}$ satisfy the following system of four differential equations:

$$
\begin{aligned}
& \partial_{\Gamma} \theta^{+\epsilon^{\prime}}=-\left(2 u_{\Gamma}^{-}(0)+u_{\Gamma}^{+}(\mu)+u_{\Gamma}^{+}(\nu)\right) \theta^{+\epsilon^{\prime}}+u_{\Gamma}^{+}(\mu) u_{\Gamma}^{+}(\nu) \sigma^{+\epsilon^{\prime}}+p_{\Gamma, \Gamma^{\prime}}^{+} \delta_{\epsilon^{\prime},+} \\
& \partial_{\Gamma} \theta^{-\epsilon^{\prime}}=-\left(2 u_{\Gamma}^{+}(0)+u_{\Gamma}^{-}(\mu)+u_{\Gamma}^{-}(\nu)\right) \theta^{-\epsilon^{\prime}}+u_{\Gamma}^{-}(\mu) u_{\Gamma}^{-}(\nu) \sigma^{-\epsilon^{\prime}}+p_{\Gamma, \Gamma^{\prime}}^{-} \delta_{\epsilon^{\prime},-} \\
& \partial_{\Gamma} \sigma^{+\epsilon^{\prime}}=\left(-2 u_{\Gamma}^{-}(0)+\frac{U_{\Gamma}^{+}(\mu) U_{\Gamma}^{-}(\mu)}{u_{\Gamma}^{+}(\mu)}+\frac{U_{\Gamma}^{+}(\nu) U_{\Gamma}^{-}(\nu)}{u_{\Gamma}^{+}(\nu)}\right) \sigma^{+\epsilon^{\prime}}-\theta^{+\epsilon^{\prime}}+\frac{U_{\Gamma}^{+}(\mu) U_{\Gamma}^{+}(\nu)}{u_{\Gamma}^{+}(\mu) u_{\Gamma}^{+}(\nu)} \theta^{-\epsilon^{\prime}} \\
& \partial_{\Gamma} \sigma^{-\epsilon^{\prime}}=\left(-2 u_{\Gamma}^{+}(0)+\frac{U_{\Gamma}^{+}(\mu) U_{\Gamma}^{-}(\mu)}{u_{\Gamma}^{-}(\mu)}+\frac{U_{\Gamma}^{+}(\nu) U_{\Gamma}^{-}(\nu)}{u_{\Gamma}^{-}(\nu)}\right) \sigma^{-\epsilon^{\prime}}-\theta^{-\epsilon^{\prime}}+\frac{U_{\Gamma}^{-}(\mu) U_{\Gamma}^{-}(\nu)}{u_{\Gamma}^{-}(\mu) u_{\Gamma}^{-}(\nu)} \theta^{+\epsilon^{\prime}}
\end{aligned}
$$

We note that the system for $\epsilon^{\prime}=+1$ and the system for $\epsilon^{\prime}=-1$ are identical except for the inhomogeneous part, that we have defined as:

$$
\begin{aligned}
p_{\Gamma, \Gamma^{\prime}}^{\epsilon^{\prime}}= & -\frac{1}{u_{\Gamma^{\prime}}^{\epsilon^{\prime}}\left(\mu^{\prime}\right)}\left(u_{\Gamma}^{\epsilon^{\prime}}(\mu)-u_{\Gamma^{\prime}}^{\epsilon^{\prime}}\left(\mu^{\prime}\right)\right)\left(u_{\Gamma}^{\epsilon^{\prime}}(\nu)-u_{\Gamma^{\prime}}^{\epsilon^{\prime}}\left(\mu^{\prime}\right)\right) C_{\Gamma, \Gamma^{\prime}}^{\epsilon^{\prime} \epsilon^{\prime}} \\
& -\frac{1}{u_{\Gamma^{\prime}}^{\epsilon^{\prime}}\left(\nu^{\prime}\right)}\left(u_{\Gamma}^{\epsilon^{\prime}}(\mu)-u_{\Gamma^{\prime}}^{\epsilon^{\prime}}\left(\nu^{\prime}\right)\right)\left(u_{\Gamma}^{\epsilon^{\prime}}(\nu)-u_{\Gamma^{\prime}}^{\epsilon^{\prime}}\left(\nu^{\prime}\right)\right) D_{\Gamma, \Gamma^{\prime}}^{\epsilon^{\prime} \epsilon^{\prime}}
\end{aligned}
$$

To exhibit explicitly the $\Gamma$ dependence, it is useful to rewrite

$$
\begin{aligned}
& p_{\Gamma, \Gamma^{\prime}}^{+}\left(\mu, \nu ; \mu^{\prime}, \nu^{\prime}\right)=\frac{1}{\bar{l}_{\Gamma}\left(\mu^{\prime}-\nu^{\prime}\right)}\left(f_{\Gamma^{\prime}}^{+}\left(\mu, \nu ; \mu^{\prime}\right) e^{-\left(\Gamma-\Gamma^{\prime}\right) u_{\Gamma^{\prime}}^{-}\left(\mu^{\prime}\right)}-f_{\Gamma^{\prime}}^{+}\left(\mu, \nu ; \nu^{\prime}\right) e^{-\left(\Gamma-\Gamma^{\prime}\right) u_{\Gamma^{\prime}}^{-}\left(\nu^{\prime}\right)}\right) \\
& p_{\Gamma, \Gamma^{\prime}}^{-}\left(\mu, \nu ; \mu^{\prime}, \nu^{\prime}\right)=\frac{1}{l_{\Gamma}\left(\mu^{\prime}-\nu^{\prime}\right)}\left(f_{\Gamma^{\prime}}^{-}\left(\mu, \nu ; \mu^{\prime}\right) e^{-\left(\Gamma-\Gamma^{\prime}\right) u_{\Gamma^{\prime}}^{+}\left(\mu^{\prime}\right)}-f_{\Gamma^{\prime}}^{-}\left(\mu, \nu ; \nu^{\prime}\right) e^{-\left(\Gamma-\Gamma^{\prime}\right) u_{\Gamma^{\prime}}^{+}\left(\nu^{\prime}\right)}\right)
\end{aligned}
$$

where we have introduced the two $\Gamma$-independent functions

$$
f_{\Gamma^{\prime}}^{\epsilon^{\prime}}\left(\mu, \nu ; \mu^{\prime}\right)=\frac{U_{\Gamma^{\prime}}^{\epsilon^{\prime}}\left(\mu^{\prime}\right)}{u_{\Gamma^{\prime}}^{\epsilon^{\prime}}\left(\mu^{\prime}\right)}\left(u_{\Gamma^{\prime}}^{\epsilon^{\prime}}\left(\mu^{\prime}\right)-u_{\Gamma^{\prime}}^{\epsilon^{\prime}}(\mu)\right)\left(u_{\Gamma^{\prime}}^{\epsilon^{\prime}}\left(\mu^{\prime}\right)-u_{\Gamma^{\prime}}^{\epsilon^{\prime}}(\nu)\right)
$$

The first step is to obtain the solutions of the above two (identical) homogeneous systems ( $\mathrm{F15})$. Remarkably, these can be constructed from the functions $U_{\Gamma}^{ \pm}(p)$ and $u_{\Gamma}^{ \pm}(p)$ using the differential equations (20). We find the four independent solutions of the homogeneous system to be: 


$$
\begin{aligned}
& \left\{\theta_{1}^{+}=n_{\Gamma} U_{\Gamma}^{+}(\mu), \quad \theta_{1}^{-}=-n_{\Gamma} U_{\Gamma}^{-}(\nu), \quad \sigma_{1}^{+}=n_{\Gamma} \frac{U_{\Gamma}^{+}(\mu)}{u_{\Gamma}^{+}(\mu)}, \quad \sigma_{1}^{-}=-n_{\Gamma} \frac{U_{\Gamma}^{-}(\nu)}{u_{\Gamma}^{-}(\nu)}\right\} \\
& \left\{\theta_{2}^{+}=n_{\Gamma} U_{\Gamma}^{+}(\mu) u_{\Gamma}^{+}(\nu), \quad \theta_{2}^{-}=n_{\Gamma} U_{\Gamma}^{-}(\nu) u_{\Gamma}^{-}(\mu), \quad \sigma_{2}^{+}=n_{\Gamma} \frac{U_{\Gamma}^{+}(\mu)}{u_{\Gamma}^{+}(\mu) u_{\Gamma}^{+}(\nu)}\right. \\
& \left.\times \quad\left(u_{\Gamma}^{+}(\nu)^{2}-U_{\Gamma}^{+}(\nu) U_{\Gamma}^{-}(\nu)\right), \quad \sigma_{2}^{-}=n_{\Gamma} \frac{U_{\Gamma}^{-}(\nu)}{u_{\Gamma}^{-}(\mu) u_{\Gamma}^{-}(\nu)}\left(u_{\Gamma}^{-}(\mu)^{2}-U_{\Gamma}^{+}(\mu) U_{\Gamma}^{-}(\mu)\right)\right\} \\
& \left\{\theta_{3}^{+}=n_{\Gamma} U_{\Gamma}^{+}(\nu), \quad \theta_{3}^{-}=-n_{\Gamma} U_{\Gamma}^{-}(\mu), \quad \sigma_{3}^{+}=n_{\Gamma} \frac{U_{\Gamma}^{+}(\nu)}{u_{\Gamma}^{+}(\nu)}, \sigma_{3}^{-}=-n_{\Gamma} \frac{U_{\Gamma}^{-}(\mu)}{u_{\Gamma}^{-}(\mu)}\right\} \\
& \left\{\theta_{4}^{+}=n_{\Gamma} U_{\Gamma}^{+}(\nu) u_{\Gamma}^{+}(\mu), \quad \theta_{4}^{-}=n_{\Gamma} U_{\Gamma}^{-}(\mu) u_{\Gamma}^{-}(\nu), \quad \sigma_{4}^{+}=n_{\Gamma} \frac{U_{\Gamma}^{+}(\nu)}{u_{\Gamma}^{+}(\mu) u_{\Gamma}^{+}(\nu)}\right. \\
& \left.\times\left(u_{\Gamma}^{+}(\mu)^{2}-U_{\Gamma}^{+}(\mu) U_{\Gamma}^{-}(\mu)\right), \sigma_{4}^{-}=n_{\Gamma} \frac{U_{\Gamma}^{-}(\mu)}{u_{\Gamma}^{-}(\mu) u_{\Gamma}^{-}(\nu)}\left(u_{\Gamma}^{-}(\nu)^{2}-U_{\Gamma}^{+}(\nu) U_{\Gamma}^{-}(\nu)\right)\right\}
\end{aligned}
$$

where $n_{\Gamma}=1 / \overline{l_{\Gamma}}=\frac{\delta^{2}}{\sinh ^{2}(\Gamma \delta)}$ and with $\partial_{\Gamma} n_{\Gamma}=-\left(u_{\Gamma}^{+}+u_{\Gamma}^{-}\right) n_{\Gamma}$.

It will be useful to consider the matrix formed by these solutions

$$
N_{\Gamma}=\left(\begin{array}{cccc}
\theta_{1}^{+} & \theta_{2}^{+} & \theta_{3}^{+} & \theta_{4}^{+} \\
\theta_{1}^{-} & \theta_{2}^{-} & \theta_{3}^{-} & \theta_{4}^{-} \\
\sigma_{1}^{+} & \sigma_{2}^{+} & \sigma_{3}^{+} & \sigma_{4}^{+} \\
\sigma_{1}^{-} & \sigma_{2}^{-} & \sigma_{3}^{-} & \sigma_{4}^{-}
\end{array}\right)
$$

From the usual properties of systems of linear equations the Wronskian $W_{\Gamma}=$ $\operatorname{det}\left[N_{\Gamma}\right]$ satisfies the simple equation, $\partial_{\Gamma} W_{\Gamma}=\operatorname{Tr}\left[M_{\Gamma}\right] W_{\Gamma}$ where $M$ is the matrix formed by the coefficients of the homogeneous part of the linear system. One can easily integrate this equation, or one can compute directly the determinant, and use the definitions (F23) to simplify the result (after a tedious calculation). Both give the same, remarkably simple, result:

$$
W_{\Gamma}=-n_{\Gamma}^{4}(\mu-\nu)^{2} \frac{U_{\Gamma}^{+}(\mu) U_{\Gamma}^{-}(\mu) U_{\Gamma}^{+}(\nu) U_{\Gamma}^{-}(\nu)}{u_{\Gamma}^{+}(\mu) u_{\Gamma}^{-}(\mu) u_{\Gamma}^{+}(\nu) u_{\Gamma}^{-}(\nu)} .
$$

Since this is not zero, this shows that the four solutions given above are linearly independent. Thus we are now in a position to write the solutions of the two linear differential systems (F15) with the inhomogeneous terms. It is found, as usual, as a linear combination of the four independent solutions (F23) of the homogeneous system:

$$
\left(\begin{array}{c}
\theta^{+\epsilon^{\prime}} \\
\theta^{-\epsilon^{\prime}} \\
\sigma^{+\epsilon^{\prime}} \\
\sigma^{-\epsilon^{\prime}}
\end{array}\right)=\sum_{i=1}^{4} \lambda_{\Gamma}^{i \epsilon^{\prime}}\left(\begin{array}{c}
\theta_{i, \Gamma}^{+}(\mu, \nu) \\
\theta_{i, \Gamma}^{-}(\mu, \nu) \\
\sigma_{i, \Gamma}^{+}(\mu, \nu) \\
\sigma_{i, \Gamma}^{-}(\mu, \nu)
\end{array}\right) \equiv N_{\Gamma} \cdot\left(\begin{array}{c}
\lambda_{\Gamma}^{1 \epsilon \epsilon^{\prime}} \\
\lambda_{\Gamma}^{2 \epsilon^{\prime}} \\
\lambda_{\Gamma}^{3 \epsilon^{\prime}} \\
\lambda_{\Gamma}^{4 \epsilon^{\prime}}
\end{array}\right)
$$

where $\lambda_{\Gamma}^{i \epsilon^{\prime}} \equiv \lambda_{\Gamma \Gamma^{\prime}}^{i \epsilon^{\prime}}\left(\mu, \nu, \mu^{\prime}, \nu^{\prime}\right)$ are the coefficients of the linear combinations. Using the standard method one finds the following equations for the coefficients:

$$
N_{\Gamma} \cdot\left(\begin{array}{c}
\partial_{\Gamma} \lambda^{1+} \\
\partial_{\Gamma} \lambda^{2+} \\
\partial_{\Gamma} \lambda^{3+} \\
\partial_{\Gamma} \lambda^{4+}
\end{array}\right)=\left(\begin{array}{c}
p_{\Gamma, \Gamma^{\prime}}^{+} \\
0 \\
0 \\
0
\end{array}\right)
$$




$$
N_{\Gamma} \cdot\left(\begin{array}{l}
\partial_{\Gamma} \lambda^{1-} \\
\partial_{\Gamma} \lambda^{2-} \\
\partial_{\Gamma} \lambda^{3-} \\
\partial_{\Gamma} \lambda^{4-}
\end{array}\right)=\left(\begin{array}{c}
0 \\
p_{\Gamma, \Gamma^{\prime}}^{-} \\
0 \\
0
\end{array}\right)
$$

The initial condition for the $\lambda_{\Gamma}^{i \epsilon^{\prime}}$ at $\Gamma=\Gamma^{\prime}$ are fixed by the initial conditions

$$
\begin{aligned}
\theta_{\Gamma^{\prime}, \Gamma^{\prime}}^{\epsilon \epsilon^{\prime}}\left(\mu, \nu ; \mu^{\prime}, \nu^{\prime}\right) & =\sum_{i=1}^{4} \lambda_{\Gamma^{\prime}, \Gamma^{\prime}}^{(i), \epsilon^{\prime}}\left(\mu, \nu ; \mu^{\prime}, \nu^{\prime}\right) \theta_{i}^{\epsilon}\left(\Gamma^{\prime}, \mu, \nu\right)=\hat{\Omega}_{\Gamma^{\prime}, \Gamma^{\prime}}^{\epsilon \epsilon^{\prime}}\left(\zeta=0, \mu, \nu ; \mu^{\prime}, \nu^{\prime}\right) \\
& =\delta_{\epsilon \epsilon^{\prime}} \frac{1}{l_{\Gamma^{\prime}}} \frac{1}{\mu^{\prime}-\nu^{\prime}}\left(U_{\Gamma^{\prime}}^{\epsilon^{\prime}}\left(\nu^{\prime}\right)-U_{\Gamma^{\prime}}^{\epsilon^{\prime}}\left(\mu^{\prime}\right)\right) \\
\sigma_{\Gamma^{\prime}, \Gamma^{\prime}}^{\epsilon \epsilon^{\prime}}\left(\mu, \nu ; \mu^{\prime}, \nu^{\prime}\right) & =\sum_{i=1}^{4} \lambda_{\Gamma^{\prime}, \Gamma^{\prime}}^{(i), \epsilon^{\prime}}\left(\mu, \nu ; \mu^{\prime}, \nu^{\prime}\right) \sigma_{i}^{\epsilon}\left(\Gamma^{\prime}, \mu, \nu\right)=\int_{0}^{\infty} d \zeta \hat{\Omega}_{\Gamma^{\prime}, \Gamma^{\prime}}^{\epsilon \epsilon^{\prime}}\left(\zeta, \mu, \nu ; \mu^{\prime}, \nu^{\prime}\right) \\
& =\delta_{\epsilon \epsilon^{\prime}} \frac{1}{l_{\Gamma^{\prime}}} \frac{1}{\mu^{\prime}-\nu^{\prime}}\left(\frac{U_{\Gamma^{\prime}}^{\epsilon^{\prime}}\left(\nu^{\prime}\right)}{u_{\Gamma^{\prime}}^{\epsilon^{\prime}}\left(\nu^{\prime}\right)}-\frac{U_{\Gamma^{\prime}}^{\epsilon^{\prime}}\left(\mu^{\prime}\right)}{u_{\Gamma^{\prime}}^{\epsilon^{\prime}}\left(\mu^{\prime}\right)}\right)
\end{aligned}
$$

Finally, we find, for $\epsilon^{\prime}=+1$, the solution of (F15) with the above initial conditions:

$$
\begin{aligned}
& \theta_{\Gamma, \Gamma^{\prime}}^{ \pm+}=\sum_{i=1}^{4} \theta_{i, \Gamma}^{ \pm}\left[\int_{\Gamma^{\prime}}^{\Gamma} d \tilde{\Gamma}\left(N_{\tilde{\Gamma}}^{-1}\right)_{i, 1} p_{\tilde{\Gamma}, \Gamma^{\prime}}^{+}+\left(N_{\Gamma^{\prime}}^{-1}\right)_{i, 1} \theta_{\Gamma^{\prime}, \Gamma^{\prime}}^{++}+\left(N_{\Gamma^{\prime}}^{-1}\right)_{i, 3} \sigma_{\Gamma^{\prime}, \Gamma^{\prime}}^{++}\right] \\
& \sigma_{\Gamma, \Gamma^{\prime}}^{ \pm+}=\sum_{i=1}^{4} \sigma_{i, \Gamma}^{ \pm}\left[\int_{\Gamma^{\prime}}^{\Gamma} d \tilde{\Gamma}\left(N_{\tilde{\Gamma}}^{-1}\right)_{i, 1} p_{\tilde{\Gamma}, \Gamma^{\prime}}^{+}+\left(N_{\Gamma^{\prime}}^{-1}\right)_{i, 1} \theta_{\Gamma^{\prime}, \Gamma^{\prime}}^{++}+\left(N_{\Gamma^{\prime}}^{-1}\right)_{i, 3} \sigma_{\Gamma^{\prime}, \Gamma^{\prime}}^{++}\right]
\end{aligned}
$$

and, for $\epsilon^{\prime}=-1$ :

$$
\begin{aligned}
& \theta_{\Gamma, \Gamma^{\prime}}^{ \pm-}=\sum_{i=1}^{4} \theta_{i, \Gamma}^{ \pm}\left[\int_{\Gamma^{\prime}}^{\Gamma} d \tilde{\Gamma}\left(N_{\tilde{\Gamma}}^{-1}\right)_{i, 2} p_{\tilde{\Gamma}, \Gamma^{\prime}}^{-}+\left(N_{\Gamma^{\prime}}^{-1}\right)_{i, 2} \theta_{\Gamma^{\prime}, \Gamma^{\prime}}^{--}+\left(N_{\Gamma^{\prime}}^{-1}\right)_{i, 4} \sigma_{\Gamma^{\prime}, \Gamma^{\prime}}^{--}\right] \\
& \sigma_{\Gamma, \Gamma^{\prime}}^{ \pm-}=\sum_{i=1}^{4} \sigma_{i, \Gamma}^{ \pm}\left[\int_{\Gamma^{\prime}}^{\Gamma} d \tilde{\Gamma}\left(N_{\tilde{\Gamma}}^{-1}\right)_{i, 2} p_{\tilde{\Gamma}, \Gamma^{\prime}}^{-}+\left(N_{\Gamma^{\prime}}^{-1}\right)_{i, 2} \theta_{\Gamma^{\prime}, \Gamma^{\prime}}^{--}+\left(N_{\Gamma^{\prime}}^{-1}\right)_{i, 4} \sigma_{\Gamma^{\prime}, \Gamma^{\prime}}^{--}\right]
\end{aligned}
$$

The next step is to evaluate $N_{\Gamma}^{-1}$, the inverse of the matrix $N_{\Gamma}$. Remarkably,the inverse admits a simple explicit form in terms of the functions $u_{\Gamma}^{ \pm}$and $U_{\Gamma}^{ \pm}$, which can be found after some tedious calculations using the form (19). It reads:

$$
N_{\Gamma}^{-1}=\frac{1}{n_{\Gamma}(\nu-\mu)}\left(\begin{array}{cccc}
-\frac{\mu}{U_{\Gamma}^{+}(\mu)} & -\frac{\nu}{U_{\Gamma}^{-}(\nu)} & \frac{u_{\Gamma}^{+}(\nu) u_{\Gamma}^{+}(\mu) u_{\Gamma}^{-}(\mu)}{U_{\Gamma}^{+}(\mu)} & \frac{u_{\Gamma}^{-}(\mu) u_{\Gamma}^{+}(\nu) u_{\Gamma}^{-}(\nu)}{U_{\Gamma}^{-}(\nu)} \\
-\frac{u_{\Gamma}^{+}(\mu)}{U_{\Gamma}^{+}(\mu)} & \frac{u_{\Gamma}^{-}(\nu)}{U_{\Gamma}^{-}(\nu)} & \frac{u_{\Gamma}^{+}(\mu) u_{\Gamma}^{+}(\nu)}{U_{\Gamma}^{+}(\mu)} & -\frac{u_{\Gamma}^{-}(\mu) u_{\Gamma}^{-}(\nu)}{U_{\Gamma}^{-}(\nu)} \\
\frac{\nu}{U_{\Gamma}^{+}(\nu)} & \frac{\mu}{U_{\Gamma}^{-}(\mu)} & -\frac{u_{\Gamma}^{+}(\mu) u_{\Gamma}^{+}(\nu) u_{\Gamma}^{-}(\nu)}{U_{\Gamma}^{+}(\nu)} & -\frac{u_{\Gamma}^{-}(\nu) u_{\Gamma}^{+}(\mu) u_{\Gamma}^{-}(\mu)}{U_{\Gamma}^{-}(\mu)} \\
\frac{u_{\Gamma}^{+}(\nu)}{U_{\Gamma}^{+}(\nu)} & -\frac{u_{\Gamma}^{-}(\mu)}{U_{\Gamma}^{-}(\mu)} & -\frac{u_{\Gamma}^{+}(\mu) u_{\Gamma}^{+}(\nu)}{U_{\Gamma}^{+}(\nu)} & \frac{u_{\Gamma}^{-}(\mu) u_{\Gamma}^{-}(\nu)}{U_{\Gamma}^{-}(\mu)}
\end{array}\right)
$$

We have thus obtained the quantities of interest, namely the $\sigma_{\Gamma, \Gamma^{\prime}}^{\epsilon \epsilon^{\prime}}\left(\mu, \nu ; \mu^{\prime} \nu^{\prime}\right)=$ $\int_{\zeta} \Omega_{\Gamma, \Gamma^{\prime}}^{\epsilon \epsilon^{\prime}}\left(\mu, \nu ; \mu^{\prime} \nu^{\prime}\right)$. The two-time probability can then be obtained from the $\sigma$ as follows. 
The Laplace transforms in the different sectors $\left(x>0, x^{\prime}>0\right),\left(x<0, x^{\prime}<\right.$ $0),\left(x<0\right.$ and $\left.x^{\prime}>0\right)$ and $\left(x>0\right.$ and $\left.x^{\prime}<0\right)$ are respectively:

$$
\begin{aligned}
\hat{P}_{\Gamma, \Gamma^{\prime}}^{++}\left(p, p^{\prime}\right) & =\int_{0}^{\infty} d x \int_{0}^{\infty} d x^{\prime} e^{-p^{\prime} x^{\prime}} e^{-p\left(x-x^{\prime}\right)} \overline{\operatorname{Prob}\left(x t, x^{\prime} t^{\prime} \mid 00\right)} \\
& =\sigma_{\Gamma, \Gamma^{\prime}}^{++}\left(\mu=p, \nu=0 ; \mu^{\prime}=p^{\prime}, \nu^{\prime}=0\right) \\
\hat{P}_{\Gamma, \Gamma^{\prime}}^{--}\left(p, p^{\prime}\right) & =\int_{-\infty}^{0} d x \int_{-\infty}^{0} d x^{\prime} e^{p^{\prime} x^{\prime}} e^{p\left(x-x^{\prime}\right)} \overline{\operatorname{Prob}\left(x t, x^{\prime} t^{\prime} \mid 00\right)} \\
& =\sigma_{\Gamma, \Gamma^{\prime}}^{--}\left(\mu=0, \nu=p ; \mu^{\prime}=0, \nu^{\prime}=p^{\prime}\right) \\
\hat{P}_{\Gamma, \Gamma^{\prime}}^{-+}\left(p, p^{\prime}\right) & =\int_{-\infty}^{0} d x \int_{0}^{\infty} d x^{\prime} e^{-p^{\prime} x^{\prime}} e^{p\left(x-x^{\prime}\right)} \overline{\operatorname{Prob}\left(x t, x^{\prime} t^{\prime} \mid 00\right)} \\
& =\sigma_{\Gamma, \Gamma^{\prime}}^{-+}\left(\mu=0, \nu=p ; \mu^{\prime}=p+p^{\prime}, \nu^{\prime}=p\right) \\
\hat{P}_{\Gamma, \Gamma^{\prime}}^{+-}\left(p, p^{\prime}\right) & =\int_{-\infty}^{0} d x^{\prime} \int_{0}^{\infty} d x e^{p^{\prime} x^{\prime}} e^{-p\left(x-x^{\prime}\right)} \overline{\operatorname{Prob}\left(x t, x^{\prime} t^{\prime} \mid 00\right)} \\
& =\sigma_{\Gamma, \Gamma^{\prime}}^{+-}\left(\mu=p, \nu=0 ; \mu^{\prime}=p, \nu^{\prime}=p+p^{\prime}\right)
\end{aligned}
$$

From these one can compute the distribution $Q\left(y, t, t^{\prime}\right)$ $=\int_{-\infty}^{+\infty} d x^{\prime} \overline{\operatorname{Prob}\left(\left(x^{\prime}+y\right), t, x^{\prime}, t^{\prime} \mid 0,0\right)}$ of the relative deplacement $y=x(t)-x\left(t^{\prime}\right)$. Its Laplace transform in the sectors $y>0$ and $y<0$ are respectively:

$$
\begin{aligned}
Q_{\Gamma, \Gamma^{\prime}}^{+}(p)= & \int_{0}^{\infty} d y e^{-p y} Q\left(y, t, t^{\prime}\right) \\
= & \int_{0}^{\infty} d x^{\prime} \int_{x^{\prime}}^{\infty} d x e^{-p\left(x-x^{\prime}\right)} \overline{\operatorname{Prob}\left(x t, x^{\prime} t^{\prime} \mid 00\right)} \\
& \quad+\int_{-\infty}^{0} d x^{\prime} \int_{0}^{\infty} d x e^{-p\left(x-x^{\prime}\right)} \overline{\operatorname{Prob}\left(x t, x^{\prime} t^{\prime} \mid 00\right)} \\
= & \sigma_{\Gamma, \Gamma^{\prime}}^{++}\left(\mu=p, \nu=0 ; \mu^{\prime}=0, \nu^{\prime}=0\right)+\sigma_{\Gamma, \Gamma^{\prime}}^{+-}\left(\mu=0, \nu=p ; \mu^{\prime}=p, \nu^{\prime}=p\right) \\
Q_{\Gamma, \Gamma^{\prime}}^{-}(p)= & \int_{-\infty}^{0} d y e^{p y} Q\left(y, t, t^{\prime}\right) \\
= & \int_{-\infty}^{0} d x^{\prime} \int_{-\infty}^{0} d x e^{p\left(x-x^{\prime}\right)} \overline{\operatorname{Prob}\left(x t, x^{\prime} t^{\prime} \mid 00\right)} \\
& +\int_{-\infty}^{0} d x \int_{0}^{\infty} d x^{\prime} e^{p\left(x-x^{\prime}\right)} \frac{\operatorname{Prob}\left(x t, x^{\prime} t^{\prime} \mid 00\right)}{\operatorname{Pr}} \\
= & \sigma_{\Gamma, \Gamma^{\prime}}^{--}\left(\mu=0, \nu=p ; \mu^{\prime}=0, \nu^{\prime}=0\right)+\sigma_{\Gamma, \Gamma^{\prime}}^{-+}\left(\mu=p, \nu=0 ; \mu^{\prime}=p, \nu^{\prime}=p\right)
\end{aligned}
$$

In order to compute these distributions, we have explicitly evaluated the sums and integrals in (F30, F32). As an example let us examine:

$$
\begin{aligned}
\sigma_{\Gamma, \Gamma^{\prime}}^{++}\left(\mu, \nu ; \mu^{\prime}, \nu^{\prime}\right)= & \sum_{i=1}^{4} \sigma_{i, \Gamma}^{+}(\mu, \nu)\left[\int_{\Gamma^{\prime}}^{\Gamma} d \tilde{\Gamma}\left(N_{\tilde{\Gamma}}^{-1}\right)_{i, 1}(\mu, \nu) p_{\tilde{\Gamma}, \Gamma^{\prime}}^{+}\left(\mu, \nu ; \mu^{\prime}, \nu^{\prime}\right)\right. \\
& \left.+\left(N_{\Gamma^{\prime}}^{-1}\right)_{i, 1}(\mu, \nu) \theta_{\Gamma^{\prime}, \Gamma^{\prime}}^{++}\left(\mu^{\prime}, \nu^{\prime}\right)+\left(N_{\Gamma^{\prime}}^{-1}\right)_{i, 3}(\mu, \nu) \sigma_{\Gamma^{\prime}, \Gamma^{\prime}}^{++}\left(\mu^{\prime}, \nu^{\prime}\right)\right]
\end{aligned}
$$

It turns out that all integrals that appear in this expression are simple exponentials. This remarkable property remains true for all other elements and is the reason why the calculation, though tedious, can be carried out explicitly for this problem. 
Explicit results for the symmetric case $\delta=0$

Here we give the explicit expression for the Laplace transform of $\overline{\operatorname{Prob}\left(x t, x^{\prime} t^{\prime} \mid 00\right)}$ in the different sectors defined in (F37). For the sector where the product $x x^{\prime}>0$, we find:

$$
\begin{aligned}
& \hat{P}_{\Gamma=\alpha \Gamma^{\prime}, \Gamma^{\prime}}^{++}\left(p=\frac{s^{2}}{\Gamma^{\prime 2}}, p^{\prime}=\frac{r^{2}}{\Gamma^{\prime 2}}\right)=\hat{P}_{\Gamma=\alpha \Gamma^{\prime}, \Gamma^{\prime}}^{--}\left(p=\frac{s^{2}}{\Gamma^{\prime 2}}, p^{\prime}=\frac{r^{2}}{\Gamma^{\prime 2}}\right) \\
& =\frac{\operatorname{coth} s}{\alpha^{2} r^{2} s}\left(1-\frac{1}{\cosh r}\right)+\frac{1}{\alpha^{2} r^{2} s \cosh (\alpha s)}\left[-\left(1-\frac{1}{\cosh r}\right)\right. \\
& \quad \cosh s \operatorname{coth} s+\left(1-\frac{r}{\sinh r}\right) \sinh s \\
& +\frac{(r \operatorname{coth} r-1)(r \operatorname{coth} r-s \operatorname{coth} s)}{\cosh r\left(s^{2}-r^{2} \operatorname{coth}{ }^{2} r\right)}\{-s \cosh s-r \operatorname{coth} r \sinh s \\
& \left.\left.+e^{-(\alpha-1) r \operatorname{coth} r}(s \cosh (\alpha s)+r \operatorname{coth} r \sinh (\alpha s))\right\}\right] \\
& +\frac{\tanh (\alpha s)}{\alpha^{2} r^{2} s}\left[\frac{r}{\sinh r}-1+s \operatorname{coth} s\left(1-\frac{1}{\cosh r}\right)\right. \\
& \left.-\frac{(r \operatorname{coth} r-1)(r \operatorname{coth} r-s \operatorname{coth} s) \tanh r}{r \cosh r}\left(1-e^{-(\alpha-1) r \operatorname{coth} r}\right)\right]
\end{aligned}
$$

For the sector where $x x^{\prime}<0$, we find:

$$
\begin{aligned}
& \hat{P}_{\Gamma=\alpha \Gamma^{\prime}, \Gamma^{\prime}}^{+-}\left(p=\frac{s^{2}}{\Gamma^{\prime 2}}, p^{\prime}=\frac{r^{2}-s^{2}}{\Gamma^{\prime 2}}\right)=\int_{-\infty}^{0} d x^{\prime} e^{r^{2} \frac{x^{\prime}}{\Gamma^{\prime 2}}} \int_{0}^{+\infty} d x e^{-s^{2} \frac{x}{\Gamma^{\prime 2}}} \frac{1}{\operatorname{Prob}\left(x t, x^{\prime} t^{\prime} \mid 00\right)} \\
& =\frac{1}{\alpha^{2}\left(s^{2}-r^{2}\right) s}\left(\left(\frac{\cosh s}{\cosh r}-1\right) \operatorname{coth} s+s-r \frac{\sinh s}{\sinh r}\right) \\
& \quad+\frac{1}{\alpha^{2}\left(s^{2}-r^{2}\right) s \cosh (\alpha s)}\left[\frac{1}{\sinh s}\left(1-\frac{\cosh s}{\cosh r}\right)\right. \\
& +\frac{(r \operatorname{coth} r-1)(r \operatorname{coth} r-s \operatorname{coth} s)}{\cosh r\left(s^{2}-r^{2} \operatorname{coth}{ }^{2} r\right)}\{-s \cosh s-r \operatorname{coth} r \sinh s \\
& \left.\left.+e^{-(\alpha-1) r \operatorname{coth} r}(s \cosh (\alpha s)+r \operatorname{coth} r \sinh (\alpha s))\right\}\right] \\
& +\frac{\tanh (\alpha s)}{\alpha^{2}\left(s^{2}-r^{2}\right) s}\left[\cosh s\left(\frac{r}{\sinh ^{2}}-\frac{s}{\sinh s}\right)+1-\frac{\cosh s}{\cosh r}\right. \\
& +\frac{(r \operatorname{coth} r-1)(r \operatorname{coth} r-s \operatorname{coth} s)}{\cosh r\left(s^{2}-r^{2} \operatorname{coth} r\right)}\{r \operatorname{coth} r \cosh s+s \sinh s \\
& \left.\left.\quad-e^{-(\alpha-1) r \operatorname{coth} r}(r \operatorname{coth} r \cosh (\alpha s)+s \sinh (\alpha s))\right\}\right]
\end{aligned}
$$

and the same expression for $\hat{P}^{-+}$.

We have performed a similar calculation in the biased case, but the corresponding full expression for the Laplace transform is too lengthy to give here. Some particular limits are discussed in the text. We give here the explicit expression of the two time correlator:

$$
\overline{\left\langle x(t) x\left(t^{\prime}\right)\right\rangle}=\frac{1}{32 \delta^{4} \sinh ^{2} \gamma^{\prime} \sinh ^{2} \gamma}\left(A\left(\gamma, \gamma^{\prime}\right)+B\left(\gamma, \gamma^{\prime}\right) e^{-\left(\gamma-\gamma^{\prime}\right) \operatorname{coth}\left(\gamma^{\prime}\right)}\right)
$$

with: 


$$
\begin{aligned}
& A\left(\gamma, \gamma^{\prime}\right)=\cosh (2 \gamma)(\sinh (2 \gamma)-\gamma)\left[\sinh \left(4 \gamma^{\prime}\right)+\sinh \left(2 \gamma^{\prime}\right)-6 \gamma^{\prime} \cosh \left(2 \gamma^{\prime}\right)\right] \\
& +\cosh (2 \gamma)\left[-\gamma^{\prime} \sinh \left(4 \gamma^{\prime}\right)+\frac{\cosh \left(4 \gamma^{\prime}\right)}{2}+4 \gamma^{\prime 2} \cosh \left(2 \gamma^{\prime}\right)-2 \gamma^{\prime} \sinh \left(2 \gamma^{\prime}\right)-\frac{1}{2}\right] \\
& +\sinh (2 \gamma)\left[-\gamma^{\prime} \cosh \left(4 \gamma^{\prime}\right)+\sinh \left(4 \gamma^{\prime}\right)-\gamma^{\prime} \cosh \left(2 \gamma^{\prime}\right)+\sinh \left(2 \gamma^{\prime}\right)+2 \gamma^{\prime}-6 \gamma^{\prime 2} \operatorname{coth} \gamma^{\prime}\right] \\
& +\gamma\left[-2 \gamma^{\prime}\left(\cosh \left(4 \gamma^{\prime}\right)+4 \cosh \left(2 \gamma^{\prime}\right)-2\right)+3\left(\sinh \left(4 \gamma^{\prime}\right)+\sinh \left(2 \gamma^{\prime}\right)\right)-12 \gamma^{\prime 2} \operatorname{coth} \gamma^{\prime}\right] \\
& +2 \gamma^{\prime} \sinh \left(6 \gamma^{\prime}\right)-2 \cosh \left(6 \gamma^{\prime}\right)-\left(6 \gamma^{\prime 2}+\frac{5}{2}\right) \cosh \left(4 \gamma^{\prime}\right)+9 \gamma^{\prime} \sinh \left(4 \gamma^{\prime}\right) \\
& +\left(6 \gamma^{\prime 2}-2\right) \cosh \left(2 \gamma^{\prime}\right)+4 \gamma^{\prime} \sinh \left(2 \gamma^{\prime}\right)-4 \gamma^{\prime 3} \operatorname{coth}\left(\gamma^{\prime}\right)+8 \gamma^{\prime 2}+\frac{13}{2} \\
& B\left(\gamma, \gamma^{\prime}\right)=\cosh \left(3 \gamma+\gamma^{\prime}\right)\left(\sinh \left(2 \gamma^{\prime}\right)-2 \gamma^{\prime}\right)^{2} \\
& +\cosh \left(\gamma+\gamma^{\prime}\right)\left(\sinh \left(2 \gamma^{\prime}\right)-2 \gamma^{\prime}\right)\left[-8 \gamma^{\prime} \cosh \left(2 \gamma^{\prime}\right)+5 \sinh \left(2 \gamma^{\prime}\right)+4\left(\gamma-\gamma^{\prime}\right) \frac{\gamma^{\prime 2}}{\sinh \left(\gamma^{\prime}\right)^{2}}\right. \\
& \left.-4 \gamma \gamma^{\prime} \operatorname{coth} \gamma^{\prime}-2 \gamma^{\prime}\right]+\sinh \left(\gamma+\gamma^{\prime}\right) 4 \gamma^{\prime 2}\left(\sinh \left(2 \gamma^{\prime}\right)-2 \gamma^{\prime}\right) \\
& +\cosh \gamma\left(\sinh \left(2 \gamma^{\prime}\right)-2 \gamma^{\prime}\right)\left[\sinh \left(3 \gamma^{\prime}\right)+4 \gamma \cosh \left(\gamma^{\prime}\right)+\sinh \left(\gamma^{\prime}\right)-4 \gamma \frac{\gamma^{\prime}}{\sinh \gamma^{\prime}}\right]
\end{aligned}
$$

\section{APPENDIX G: DISORDER AVERAGED PROBABILITY DISTRIBUTION FOR A FINITE SIZE SYSTEM}

In this Appendix we consider a finite size system $0<x<L$ using results of Sections $\nabla \Pi \mathrm{A}$ and $\Pi \mathrm{C} 3$. We start with a RR system, i.e., reflecting boundaries on each end.

We will denote by $b=2 k+2, k=0,1,2, . .+\infty$, the number of renormalized bonds in the system. The disorder averaged distribution can be written as a sum:

$$
\overline{P_{0 L}\left(x, t \mid x_{0}, 0\right)}=\sum_{k=0}^{k=+\infty} \sum_{N=1}^{2 k+2} P_{2 k+2}^{N, L}\left(x, t \mid x_{0}, 0\right)
$$

where $P_{2 k+2}^{N, L}\left(x, t \mid x_{0}, 0\right)$ corresponds to the contribution of the case where the starting point $x_{0}$ is on the $N^{t h}$ bond (see Figure 8). One must distinguish between $N=2 n+1$

odd, when the particle starts on a descending bond and $x>x_{0}$ and $N=2 n+2$ even when it starts on an ascending bond and $x<x_{0}$. Thus, in addition to (G1) above we will also be interested in the explicit decomposition:

$$
\begin{aligned}
& P_{0 L}\left(x, t \mid x_{0}, 0\right)=\theta\left(x-x_{0}\right) P_{0 L}^{+}\left(x, t \mid x_{0}, 0\right)+\theta\left(x_{0}-x\right) P_{0 L}^{-}\left(x, t \mid x_{0}, 0\right) \\
& \theta\left(x-x_{0}\right) P_{0 L}^{+}\left(x, t \mid x_{0}, 0\right)=\sum_{k=0}^{k=+\infty} \sum_{n=0}^{k} P_{2 k+2}^{2 n+1, L}\left(x, t \mid x_{0}, 0\right)
\end{aligned}
$$

One has (see Figure 8), for $n=0, . . k$ :

$$
P_{2 k+2}^{2 n+1, L}\left(x, t \mid x_{0}, 0\right)=\left\langle\delta\left(x-\sum_{i=1}^{2 n+1} l_{i}\right) \theta\left(\sum_{i=1}^{2 n} l_{i}<x_{0}<x\right)\right\rangle_{2 k+2}
$$


where $\langle. .\rangle_{2 k+2}$ denotes the average over the $2 k+2$ bond sector of the finite size measure $\mathrm{RR}$ in (198). There is a similar formula for an even number of initial bonds. Throughout we will define Laplace transforms $x \rightarrow p, x_{0} \rightarrow p_{0}, L \rightarrow q$ as follows:

$$
P_{2 k+2}^{N}\left(p, p_{0}, q\right)=\int_{0}^{+\infty} d L \int_{0}^{L} d x \int_{0}^{L} d x_{0} e^{-\left(p x+p_{0} x_{0}+q L\right)} P_{2 k+2}^{N, L}\left(x, t \mid x_{0}, 0\right) .
$$

One finds:

$$
\begin{aligned}
& P_{2 k+2}^{2 n+1}\left(p, p_{0}, q\right)=\frac{\bar{l}_{\Gamma}}{p_{0}} E_{p+p_{0}+q}^{+}\left(P_{p+p_{0}+q}^{-} P_{p+p_{0}+q}^{+}\right)^{n-1} P_{p+p_{0}+q}^{-}\left(P_{q+p}^{+}-P_{p+p_{0}+q}^{+}\right)\left(P_{q}^{-} P_{q}^{+}\right)^{k-n} E_{q}^{-} \\
& P_{2 k+2}^{2 n+2}\left(p, p_{0}, q\right)=\frac{\bar{l}_{\Gamma}}{p_{0}} E_{p+p_{0}+q}^{+}\left(P_{p+p_{0}+q}^{-} P_{p+p_{0}+q}^{+}\right)^{n}\left(P_{q}^{-}-P_{p_{0}+q}^{-}\right) P_{q}^{+}\left(P_{q}^{-} P_{q}^{+}\right)^{k-n-1} E_{q}^{-}
\end{aligned}
$$

the first formula being valid for $1 \leq n \leq k$ (and $k \geq 1$ ) and the second for $0 \leq n \leq$ $k-1$ (and $k \geq 1$ ). Finally for the two edge bonds one has:

$$
\begin{aligned}
& P_{2 k+2}^{1}\left(p, p_{0}, q\right)=\frac{\bar{l}_{\Gamma}}{p_{0}}\left(E_{q+p}^{+}-E_{p+p_{0}+q}^{+}\right)\left(P_{q}^{-} P_{q}^{+}\right)^{k} E_{q}^{-} \\
& P_{2 k+2}^{2 k+2}\left(p, p_{0}, q\right)=\frac{\bar{l}_{\Gamma}}{p_{0}} E_{p+p_{0}+q}^{+}\left(P_{p+p_{0}+q}^{-} P_{p+p_{0}+q}^{+}\right)^{k}\left(E_{q}^{-}-E_{p_{0}+q}^{-}\right)
\end{aligned}
$$

for any $k$. Resumming and using the identities (199) yields:

$$
\begin{aligned}
P^{+}\left(p, p_{0}, q\right)= & \frac{1}{p_{0} q} \frac{E_{q+p}^{+}}{E_{q}^{+}}+\frac{1}{\bar{l}_{\Gamma} p_{0} q\left(p+p_{0}+q\right)} \frac{P_{p+p_{0}+q}^{-} P_{p+q}^{+}-1}{E_{p+p_{0}+q}^{-} E_{q}^{+}} \\
P\left(p, p_{0}, q\right)= & \frac{1}{\bar{l}_{\Gamma} p_{0} q\left(p+p_{0}+q\right)} \frac{P_{p+p_{0}+q}^{-} P_{p+q}^{+}-P_{p_{0}+q}^{-} P_{q}^{+}}{E_{p+p_{0}+q}^{-} E_{q}^{+}}+\frac{1}{p_{0} q} \frac{E_{q+p}^{+}}{E_{q}^{+}} \\
& \quad-\frac{1}{p_{0}\left(p+p_{0}+q\right)} \frac{E_{p_{0}+q}^{-}}{E_{p+p_{0}+q}^{-}}
\end{aligned}
$$

A simpler expression holds at coinciding points:

$$
\int_{0}^{+\infty} d L \int_{0}^{+\infty} d x_{0} e^{-p_{0} x_{0}-q L} \overline{P_{0 L}\left(x_{0}, t \mid x_{0}, 0\right)}=\frac{1}{\bar{l}_{\Gamma} q\left(p_{0}+q\right) E_{p_{0}+q}^{-} E_{q}^{+}}
$$

A similar calculation in the case of absorbing boundaries (AA case) gives:

$$
P\left(p, p_{0}, q\right)=\frac{\left(P_{q+p}^{+} P_{q}^{-}-P_{p+p_{0}+q}^{+} P_{p_{0}+q}^{-}\right)}{\bar{l}_{\Gamma} p_{0} q\left(p+p_{0}+q\right) E_{p+p_{0}+q}^{+} E_{q}^{-}}
$$

as well as the semi-infinite limit $L=\infty$ with an absorbing boundary at $x=0$

$$
P_{0 \infty}\left(p, p_{0}\right)=\frac{\left(P_{p}^{+}-P_{p+p_{0}}^{+} P_{p_{0}}^{-}\right)}{\bar{l}_{\Gamma} p_{0}\left(p+p_{0}\right) E_{p+p_{0}}^{+}}
$$

At coinciding points in semi-infinite system this becomes simply 


$$
\int_{0}^{+\infty} d x_{0} e^{-p_{0} x_{0}} \overline{P\left(x_{0}, t \mid x_{0}, 0\right)}=\frac{P_{p_{0}}^{+}}{\bar{l}_{\Gamma} p_{0} E_{p_{0}}^{+}}
$$

In the RA case the result is

$$
P\left(p, p_{0}, q\right)=\frac{\left(E_{q+p}^{+}\right) P_{q}^{-}}{p_{0} q E_{q}^{-}}+\frac{\left(P_{q}^{-} P_{p+p_{0}+q}^{-} P_{q+p}^{+}-P_{q+p_{0}}^{-}\right)}{\bar{l}_{\Gamma} p_{0} q\left(p+q+p_{0}\right) E_{p+p_{0}+q}^{-} E_{q}^{-}}
$$

The AR case being obtained by the global exchange of + and - as well as $x \rightarrow L-x$ and $x_{0} \rightarrow L-x_{0}$ (i.e $p \rightarrow-p, p_{0} \rightarrow-p_{0}$ and $q \rightarrow q+p+p_{0}$ ). 


\section{REFERENCES}

[1] E. Vincent, J. Hammann and M. Ocio; in 'Recent progress in Random Magnets', ed. D. H. Ryan, World Scientific, Singapore (1992).

[2] see e.g. S.R. Anderson Phys. Rev B 368435 (1987).

[3] D.S. Fisher, D.A. Huse Phys. Rev. B 38373 (1988).

[4] G. Blatter, M. V. Feigel'man, V. B. Geshkenbein, A. I Larkin and V. M. Vinokur, Review of Modern Physics 6611251994.

[5] T. Giamarchi and P. Le Doussal Phys. Rev. B52 1242 (1995).

[6] L. F. Cugliandolo and J. Kurchan; Phys. Rev. Lett. 71, 173 (1993) and J. Phys. A27, 5749 (1994).

[7] H. Kinzelbach and H. Horner; J. Phys. I (France) 3, 1329 (1993), ibid 3, 1901 (1993).

[8] L. Cugliandolo, P. Le Doussal , Phys. Rev. E 531525 (1996), L. Cugliandolo, J. Kurchan, P. Le Doussal, Phys. Rev. Lett. 762390 (1996).

[9] J. Kurchan and L. Laloux J. Phys. A Math. Gen. 291929 (1996).

[10] A. J. Bray Adv. Phys. 43357 (1994).

[11] Y. G. Sinai Theor. Probab. Its Appl. 27247 (1982).

[12] H. Kesten, Physica 138 A 299 (1986).

[13] B. Derrida, J. Stat. Phys. 31433 (1983).

[14] J. P. Bouchaud, A. Comtet, A. Georges, P. Le Doussal Europhys. Lett. 3653 (1987) and Ann. Phys. 201285 (1990).

[15] A. Comtet, J. Desbois and C. Monthus Ann. Phys. 239312 (1995). C. Monthus et al. Phys. Rev. E 54231 (1996).

[16] L. Laloux and P. Le Doussal cond-mat/9705249, Phys. Rev. E 576296 (1998) and references therein.

[17] M. Feigelman and V. M. Vinokur, J. Phys. France 491731 (1988).

[18] D.S. Fisher, P. Le Doussal and C. Monthus, cond-mat/9710270, Phys. Rev. Lett. 803539 (1998).

[19] C. Dasgupta and S.K. Ma Phys. Rev. B 221305 (1980).

[20] D.S. Fisher, Phys. Rev. B 503799 (1994).

[21] D.S. Fisher, Phys. Rev. B 516411 (1995).

[22] D.S. Fisher and A.P. Young, Preprint cond-mat/9802246.

[23] R.A. Hyman and K. Yang, Phys. Rev. Lett. 781783 (1997).

[24] C. Monthus, O. Golinelli and T. Jolicoeur, Phys. Rev. Lett 793254 (1997).

[25] H. Kesten, M. Koslov, F. Spitzer, Compos. Math. 30145 (1975).

[26] A. O. Golosov Soviet. Math. Dokl. 2819 (1983).

[27] A. O. Golosov, Commun. of the Moscow Math. Soc. 199 (1985).

[28] For review see H. Tanaka, Proceedings of Int. Conf. Math. 1994.

[29] for review see e.g. Refs [14,35,34,32 and references therein.

[30] D.S. Fisher, P. Le Doussal, C. Monthus in preparation.

[31] P. Le Doussal, C. Monthus in preparation.

[32] L. Balents and M. P. A. Fisher cond-mat/9706069.

[33] A. Comtet, A. Georges, P. Le Doussal, Physics Lett. B 208487 (1988).

[34] A. Comtet and C. Texier, cond-mat 9707313 (1997).

[35] R. H. McKenzie, Phys. Rev. Lett. 774804 (1996).

[36] F. Igloi and H. Rieger cond-mat/9709260 (1997) and references therein. 
[37] A. Maritan Phys. Rev. Lett. 62, 2845 (1989); A. Maritan, G. Sartoni and A.L. Stella , Phys Rev. Lett 71, 1027 (1993). A. Giacometti,A. Maritan and H. Nakanishi, J. Stat. Phys. 75, 669 (1994). A. Giacometti and A. Maritan Phys. Rev.E 49,227 (1994). J.R. Banavar and A. Maritan Phys. Rev. E47, 769 (1993). [38] A.J. Bray, B. Derrida and C. Godreche Europhys. Lett. 27175 (1994). A.D. Rutenberg and A.J. Bray Phys. Rev. E 501900 (1994). A.J. Bray and B. Derrida Phys. Rev. E 51 R1633 (1995). B. Derrida, C. Godreche and I. Yekutieli Europhys. Lett. 12385 (1990) and Phys. Rev. A 446241 (1991).

[39] F. Igloi and H. Rieger cond-mat/9804316 (1998).

[40] A. Comtet and D. Dean, cond-mat/9809111 (1998).

[41] J. Chave and E. Guitter, cond-mat/9809087 (1998).

[42] M. Stephen, Rutgers Preprint 1998.

[43] see e.g. Karlin and Altschul, Proc. Nat'l Acad. Sci. (1990) and P. Bermel, R. Bundschuh, R. Olsen and T. Hwa (in preparation) and references therein.

[44] A. Comtet, C. Monthus and M. Yor cond-mat/9601014.

[45] see formulae (43-50) in [16]) given that in the small $\mu$ limit $\left(t-t^{\prime}\right)^{\mu} \sim t^{\mu}(1-$ $\left.\exp \left(\frac{1}{\mu}\left(\gamma-\gamma^{\prime}\right)\right)\right)^{\mu}$ can be replaced by $t^{\mu}$ in the exponential of (168).

[46] S.H. Noskowicz and I.G. Goldhirsh, Phys. Rev. A42 , 4, 2047 (1990).

[47] P. Le Doussal, Phys. Rev. Lett. 623097 (1989).

[48] D.S. Fisher, in preparation.

[49] Mau, Motrunich, D. A. Huse, D.S. Fisher in preparation and Bull.Am.Phys.Soc. 44 (March 1999) in press. 